\title{
The ecology of ditches
}

A modelling perspective

Luuk P.A. van Gerven 


\section{Thesis committee}

\section{Promotor}

Prof. Dr W.M. Mooij

Professor of Aquatic Foodweb Ecology

Wageningen University, Netherlands Institute of Ecology

\section{Co-promotors}

Dr J.J.M. de Klein

Assistant Professor, Aquatic Ecology and Water Quality Management Group Wageningen University

Dr J.H. Janse

Senior scientist

The Netherlands Environmental Assessment Agency (PBL), Bilthoven

\section{Other members}

Prof. Dr C. Kroeze, Wageningen University

Dr S. Hilt, IGB, Berlin, Germany

Prof. Dr M.G. Rietkerk, Utrecht University

Prof. Dr P.F.M. Verdonschot, University of Amsterdam

This research was conducted under the auspices of the C.T. de Wit Graduate School for Production Ecology and Resource Conservation (PE\&RC) 


\title{
The ecology of ditches
}

\section{A modelling perspective}

\author{
Luuk P.A. van Gerven
}

\section{Thesis}

submitted in fulfilment of the requirements for the degree of doctor at Wageningen University by the authority of the Rector Magnificus

Prof. Dr A.P.J. Mol, in the presence of the

Thesis Committee appointed by the Academic Board to be defended in public on Wednesday 16 November 2016 at 1.30 p.m. in the Aula 
Luuk P.A. van Gerven

The ecology of ditches. A modeling perspective,

146 pages.

PhD thesis, Wageningen University, Wageningen, NL (2016)

With references, with summaries in English and Dutch

ISBN 978-94-6257-931-6

DOI $10.18174 / 390133$ 


\section{Contents}

1 General introduction 1

2 Competition for light and nutrients in layered communities of aquatic $\begin{array}{ll}\text { plants } & 15\end{array}$

$3 \mathrm{~N}_{2}$-fixation in aquatic ecosystems does not axiomatically lead to $\mathbf{P}$ limitation 39

4 How regime shifts in connected aquatic ecosystems are affected by the typical downstream increase of water flow

5 Advantages of concurrent use of multiple software frameworks in water quality modelling using a database approach

6 General Discussion $\quad 93$

$\begin{array}{ll}\text { References } & 105\end{array}$

$\begin{array}{ll}\text { Summary } & 121\end{array}$

$\begin{array}{ll}\text { Samenvatting } & 125\end{array}$

$\begin{array}{ll}\text { Dankwoord } & 129\end{array}$

$\begin{array}{ll}\text { About the author } & 133\end{array}$

$\begin{array}{ll}\text { Publications } & 135\end{array}$ 



\section{Chapter 1}

\section{General introduction}

\subsection{The origin and ecology of ditches}

\section{On the origin of ditches}

A famous English saying states that "God created the world but the Dutch created Holland". It illustrates the rich history of the Netherlands in water management. Fighting water has been a core business since ages and shaped large parts of the current Dutch landscape. Dutch water management set off around 500 B.C. when our ancestors began to construct artificial hills (mounds) as a safeguard for the sea. From 800 A.D. onwards, peat marshes that covered large parts of the Netherlands were turned into agricultural areas to feed the increasing human population. To do so, the water table needed to be lowered to create dry land for farms and pastures, which was done by digging drainage ditches. At that moment one did not know yet that draining these peat lands would initiate a major future problem. Namely, the extraction of water led to the decomposition and subsidence of peat, causing the land to subside with velocities up to $2 \mathrm{~cm}$ per year (Schothorst 1977). Therefore, the Netherlands nowadays harbors many polder areas, which lie lower than the surrounding rivers, often even below sea level, and are enclosed by dikes (Fig. 1.1). As a consequence, water can only leave a polder by being pumped to the higher located surrounding rivers. For that wind mills were used in the old days and nowadays one relies on pumping station powered by electricity, fuel or steam.

Not all polder areas originated from draining peat marshes. Some arose from reclaiming land from the sea and from turning lakes into land. Nowadays, polder areas cover almost half of the Netherlands and are mainly situated in the west and north. They are characterized by dense networks of drainage 


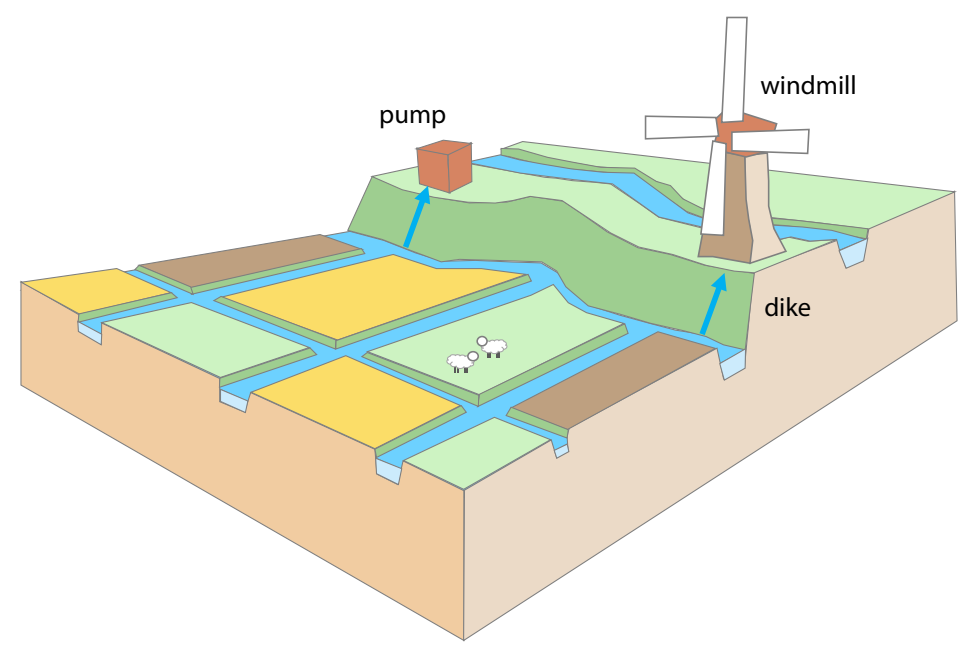

Figure 1.1. A typical polder system.

ditches. The total length of these ditches is estimated on $300.000 \mathrm{~km}$ (Verdonschot et al. 2012), which almost equals the distance from the earth to the moon. Ditches are typically several meters wide and less than 1.5 meter deep. Most are mowed each year and dredged every 5 to 10 years, which is done to ensure their discharging capacity. Ditches not only drain water, but also supply water to the agricultural land, especially during dry summer periods. Then, water from the surrounding rivers is let into the polder, which potentially reverses the ditch's flow direction.

\section{Ecology of ditches}

Ditches provide many valuable goods and services to society, not only by enabling agricultural practices (their hydrological and main function) and by adding to the aesthetic value of the Dutch landscape, but also ecologically by serving as ecological corridors and supporting a high biodiversity (Armitage et al. 2003). Therefore, it is important to ensure the ecological quality of ditches. The ecological quality is closely related to the type of water plants that grow in a ditch. Water plants have a high growth potential in ditches, as ditches provide an optimal light climate given their shallowness and situation in the open landscape (no shading). This can give rise to a diverse community of water plants, which serve as food or provide habitat for other aquatic life and thereby promote biodiversity (Higler and Verdonschot 1989; Scheffer et al. 1984). 


\section{Eutrophication}

The ecological quality of ditches is threatened by eutrophication, the overenrichment of aquatic ecosystems with nutrients. Eutrophication is a worldwide phenomenon and is caused by enhanced nutrient input by human activities. It concerns point sources from urban or industrial areas and diffuse emissions related to agricultural activities (Carpenter et al. 1998). Ditches particularly receive nutrients by diffuse emissions from the surrounding agricultural fields. Especially the excessive use of fertilizers from the 1970's onwards resulted in a strong increase of nutrient input to ditches with dramatic implications for the ecological quality of ditches (Janse 1998).

\section{Dominance of free-floating plants}

Due to eutrophication, the water plant community in many ditches changed from highly diverse to less diverse. Plant communities consisting of multiple species were replaced by monocultures of more productive species like the submerged water weed (Elodea spp.), and these species were in turn replaced by monocultures of free-floating plants such as duckweeds (Lemna sp.) and water ferns (Azolla sp.). This succession of water plants with increasing nutrient availability was confirmed in experimental ditches (Portielje and Roijackers 1995), mesocosm experiments (Netten et al. 2010), laboratory experiments (Roijackers et al. 2004), and model studies (Janse 1998; Scheffer et al. 2003).

Free-floating plants, often considered as the endpoint of eutrophication, threaten the biodiversity and ecological functioning of a ditch, especially if they form dense mats. Underneath such mats, the water becomes too dark for submerged plants to persist and photosynthesize (Morris et al. 2004). As a consequence, the water gets low in oxygen, also because the floating plants release their produced oxygen directly to the atmosphere and hamper the oxygen supply from air to water (reaeration). In the worst case this leads to anoxia, having dramatic consequences for macroinvertebrates and fish (Portielje and Roijackers 1995; Verdonschot and Verdonschot 2014).

The invasion by dense mats of free-floating plants is not only an issue in Dutch ditches, but is also frequently observed in temperate ponds and even in tropical lakes, making it a worldwide problem. Some tropical lakes for example, suffer from the invasion of water hyacinth (Eichhornia crassipes), giant salvinia (Salvinia molesta) and water lettuce (Pistia stratiotes), resulting in dark anoxic conditions that have a profound negative impact on fisheries (Mehra et al. 1999; Oliver 1993). 


\subsection{Understanding and predicting the ecology of ditches}

\section{Regime shifts and consequences for management}

For ecosystem management on ditches, it is important to know how the regime shift from submerged to floating plants evolves. Is it a gradual transition or an abrupt shift, and how difficult is it to restore a ditch in its original state once it became floating-plant dominated? Theory on regime shifts predicts that an ecosystem can respond in different ways to gradually changing conditions like eutrophication (Scheffer et al. 2001). It can respond smoothly in the sense that there is a gradual transition from one state to the other (Fig. 1.2a) or more abruptly (Fig. 1.2b). When the ecosystem response is most abrupt (Fig. 1.2c) the ecosystem may show alternative stable states. Then the ecosystem can either be in one state or in another contrasting state for a certain range of conditions. This has drastic consequences for ecosystem management, as it makes it much harder to restore the ecosystem in its original state once it has shifted to the contrasting state. For ditches this would mean that once a ditch shifts from dominance of submerged plants to freefloating plants at a certain nutrient loading, the nutrient loading has to be reduced much further to restore the ditch in its original state.
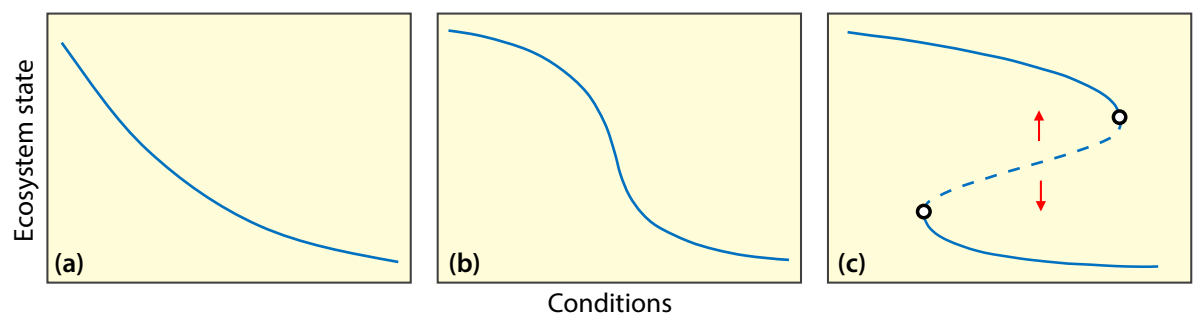

Figure 1.2. Possible ways of how an ecosystem can respond to gradually changing conditions after Scheffer et al. (2001): (a) gradual, (b) more abruptly or (c) by "folding backwards" implying alternative stable states.

The concept of alternative stable states has become an influential framework in ecology to explain the behavior of all kinds of ecosystems, like coral reefs (Knowlton 1992), woodlands (Dublin et al. 1990), deserts (Rietkerk et al. 1997) and oceans (Hare and Mantua 2000). Shallow lakes have become the archetypal example of ecosystems with alternative stable states (Scheffer and Van Nes 2007). Alternative stable states arise from self-stabilizing mechanisms that make a state resistant to a switch to the contrasting state. For example, the clear state of a lake maintains itself due to the clarifying effect 
of submerged vegetation by inhibiting sediment resuspension, whereas the turbid state is maintained by algae that keep the light availability too low for submerged vegetation to persist (Fig. 1.3a).

For ditches, however, it is not clear yet if they possess alternative stable states. When constructing a feedback diagram for ditches (Fig. 1.3b), similar to the one for lakes, you would expect that ditches also exhibit alternative stable states. The state of submerged-plant dominance could enhance itself by keeping the nutrient levels too low for floating plants to invade, whereas the floating-plant dominated state could maintain itself by keeping the light level too low for submerged plants to establish, due to shading.
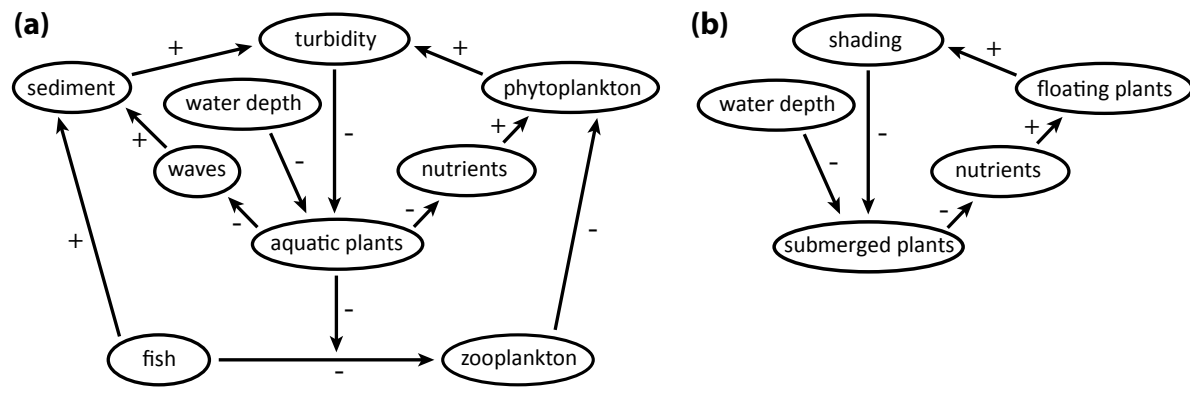

Figure 1.3. Possible feedback mechanisms in (a) shallow lakes, after Scheffer et al. (1993), and in (b) ditches.

\section{Ecological models}

To better understand and predict the response of ecosystems to changing conditions, a huge variety of process-based ecological models has been developed. One of the first ecological models developed was the Lotka-Volterra predator-prey model (Lotka 1920; Volterra 1928). Over time, the number of models as well as their complexity remarkably increased, especially since the onset of 'the computer era' (Janssen et al. 2015). Currently, there are two main types of models, representing essentially different worlds. On one hand there are the simple minimal models, embraced by theoreticians. On the other hand there are the complex ecosystem models, popular among applied ecologists and ecosystem managers. Both worlds have their pros and cons.

Simple minimal models typically aim at understanding a certain phenomenon of the ecosystem that cannot easily be understood intuitively (Scheffer and Beets 1994). They usually consist of only a few differential equations that each describe the processes by which a certain state variable (e.g. water 
plant biomass) changes in time. Given their explanatory power, simple models contributed much to ecological theory. However, their predictive power is limited as simple models often neglect important aspects of the ecosystem, which also makes it difficult to test and validate them with empirical data (Van Nes 2002).

Complex ecosystem models are typically designed for prediction, and therefore intend to include all ecological key processes that affect the ecosystem's behaviour. This makes it possible to quantify the relative importance of a certain process. Complex models are often criticized for being hard to understand: their results are too incomprehensible to test hypotheses and contribute to ecological theory (Grimm 1994). Also, complex models are difficult to validate, because empirical data can be fitted well with very different sets of parameter values and even with different model structures (Simons and Lam 1980). Thus, a good model fit may be obtained for the wrong reasons, hampering the predictive capabilities of the model (Rykiel 1996).

\section{Competition theory}

An example of ecological theory whose development is strongly based on simple models is mechanistic competition theory. It plays an important role in this thesis, as the ecology of ditches strongly depends on the water plant community, whose structure is shaped by the competition of water plants for nutrients and light. Tilman (1980) was among the first to mechanistically describe the competition of species (consumers) for nutrients (resources), and the resulting consumer-resource interactions. His theory, however, does not apply to light. This is because light is a fundamentally different resource compared to nutrients, as light cannot be recycled and is never distributed homogeneously over the water column as it declines with depth and biomass due to light attenuation (Weissing and Huisman 1994).

Huisman and Weissing (1995) extended mechanistic competition theory by adding light as a new resource. Box 1.1 explains the main insights of their work. It shows how the competition outcome depends on how much nutrients and light the species require and consume. The species with the lowest requirements is the best competitor and wins the competition, as it can deplete the available light and nutrients to a level too low for the other species to persist. However, when there are trade-offs in competitive strength, so when one species is a better competitor for light and the other species is better at nutrients, the species potentially coexist or represent alternative stable states, depending on how much light and nutrients they consume. 


\section{Box 1.1 Species competing for nutrients and light: who wins?}

Consider two species that compete for a nutrient and light in a well-mixed water column. When assuming that their biomass is uniformly distributed with depth $s$ - where the total biomass $W$ of species $i$ equals its biomass density $\omega$ times the covered total depth of the water column $z\left(W_{i}=\omega_{i} z\right)-$ and that their growth rate $p$ increases with both nutrient concentration $R$ and light intensity $I$, the biomass dynamics read (Huisman and Weissing 1995):

$$
\frac{\mathrm{d} W_{i}}{\mathrm{~d} t}=\int_{0}^{z}\left(p_{i}(I, R)-l_{i}\right) \omega_{i} \mathrm{~d} s=P_{i}-l_{i} W_{i}
$$

where $l$ is the species' loss rate and $P$ is the biomass production. Integrating over depth is needed because the light intensity $I$ decreases with depth due to the background extinction $K_{b g}$ of the water, and due to attenuation by biomass, with light attenuation coefficient $k$. According to Lambert Beer's law I reads

$$
I=I_{i n} e^{-\left(\sum k_{i} \omega_{i}+K_{b g}\right) s},
$$

where $I_{i n}$ is the incoming light intensity. The light intensity at the bottom $I_{\text {out }}$ then denotes

$$
I_{\text {out }}=I_{\text {in }} e^{-\left(\sum k_{i} W_{i}+K_{\text {bg }} z\right)} .
$$

These light equations show that the species interact via light absorption. They also interact via nutrient consumption, which affects the nutrient concentration $R$ in the water:

$$
\frac{\mathrm{d} R}{\mathrm{~d} t}=D\left(R_{i n}-R\right)-\frac{1}{z} \sum_{j=1}^{n} c_{j} P_{j},
$$

where the first term represents the in- and outflow of nutrients governed by dilution rate $D$ and the concentration of the incoming water $R_{i n}$, and $c$ denotes the species' nutrient to biomass ratio.

The competition outcome can now be graphically predicted by comparing the zero net growth isoclines (ZNGI) of both species. The ZNGI gives combinations of $R$ and $I_{\text {out }}$ at which a species can persist (see solid lines in Fig. 1.4), and can be derived by solving eq. 1.1 for equilibrium $\left(\mathrm{d} W_{i} / \mathrm{d} t=0\right)$. It has a curved shape, because with increasing $R$ the species gains biomass, which leads - for a certain $I_{\text {in }}$ as in Fig. 1.4 - to a lower $I_{\text {out }}$ due to more light attenuation. The ZNGI also shows to which extent a species can deplete the nutrient and light level, and therefore represents the competitive strength of a species. The best competitor for light is the species that can deplete $I_{\text {out }}$ to the lowest level, too low for the other species to persist. Similarly, the best nutrient competitor can deplete $R$ to the lowest level. When a species is the best competitor for both light and 
nutrients it will therefore always win by competitive exclusion.

When there are trade-offs in competitive strength, so when one species is a better nutrient competitor and the other a better light competitor (making their ZNGI's intersect), different competition outcomes are possible (Fig. 1.4). The outcome then depends on the resource supply point $\left(R=R_{i n}, I_{\text {out }}=I_{\text {in }} e^{K_{b g} z}\right)-$ the highest possible resource levels - and the species' consumption vectors that represent their light attenuation and nutrient uptake. The species coexist when the supply point falls in the region bounded by the consumption vectors, at least when each species consumes more of the resource that most limits its growth (Fig. 1.4a). Otherwise they show alternative states: either species 1 or species 2 wins, depending on who established first (Fig. 1.4b).

The graphical isocline approach of Fig. 1.4 can also be used to predict the competition outcome of species that compete for nutrients only (Tilman 1982), then with two nutrients on the axes. A major difference, however, is that for nutrients the competitive strength of a species is irrespective of the resource supply. For light this is not the case, as the incoming light intensity $I_{i n}$ shifts the ZNGI of a species and therefore changes its competitive strength.
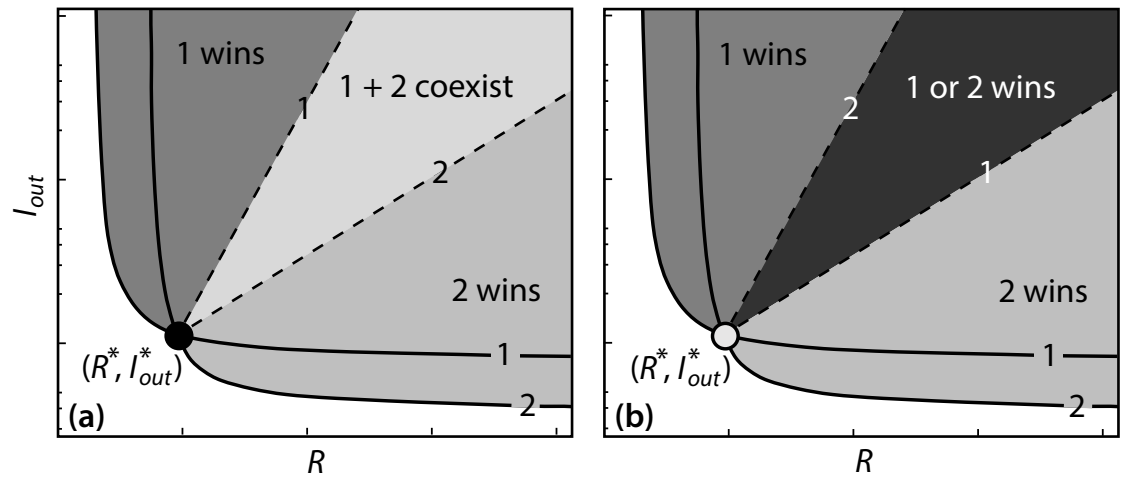

Figure 1.4. Competition outcome of two species competing for light and a nutrient in a mixed water column for a certain $I_{i n}$, after Huisman and Weissing (1995). It shows the ZNGI's (solid lines) and consumption vectors (dashed lines) of species 1 and 2 . The shaded regions give the competition outcome for resource supply points that fall within that region. In (a) and (b) the consumption vectors of both species are reversed, leading in (a) to a stable coexistence equilibrium $\left(R^{*}, I_{\text {out }}^{*}\right)$ implying coexistence, and in (b) to an unstable equilibrium implying alternative stable states.

\section{PCDitch}

Next to simple models, you can use complex models to simulate the ecology of ditches. In this thesis I use the complex ecosystem model PCDitch to 
do so (Janse 1998, 2005). This model, developed in the 1990s, describes the water plant community in ditches and includes the main key processes that shape this community, including oxygen levels and nutrient cycling processes in water and sediment. PCDitch considers six different water plant types that compete for nutrients and light (Fig. 1.4). Each type has its own competition strategy related to its growth form. For example, plants with floating leaves benefit from being the first to get the light, whereas rooted submerged plants have an advantage considering nutrients, as they not only have access to nutrients in the water but also in the sediment. The model predicts the competition outcome by computing how the water plants' daily biomass changes throughout the year, given environmental conditions like light, temperature and nutrient loading.

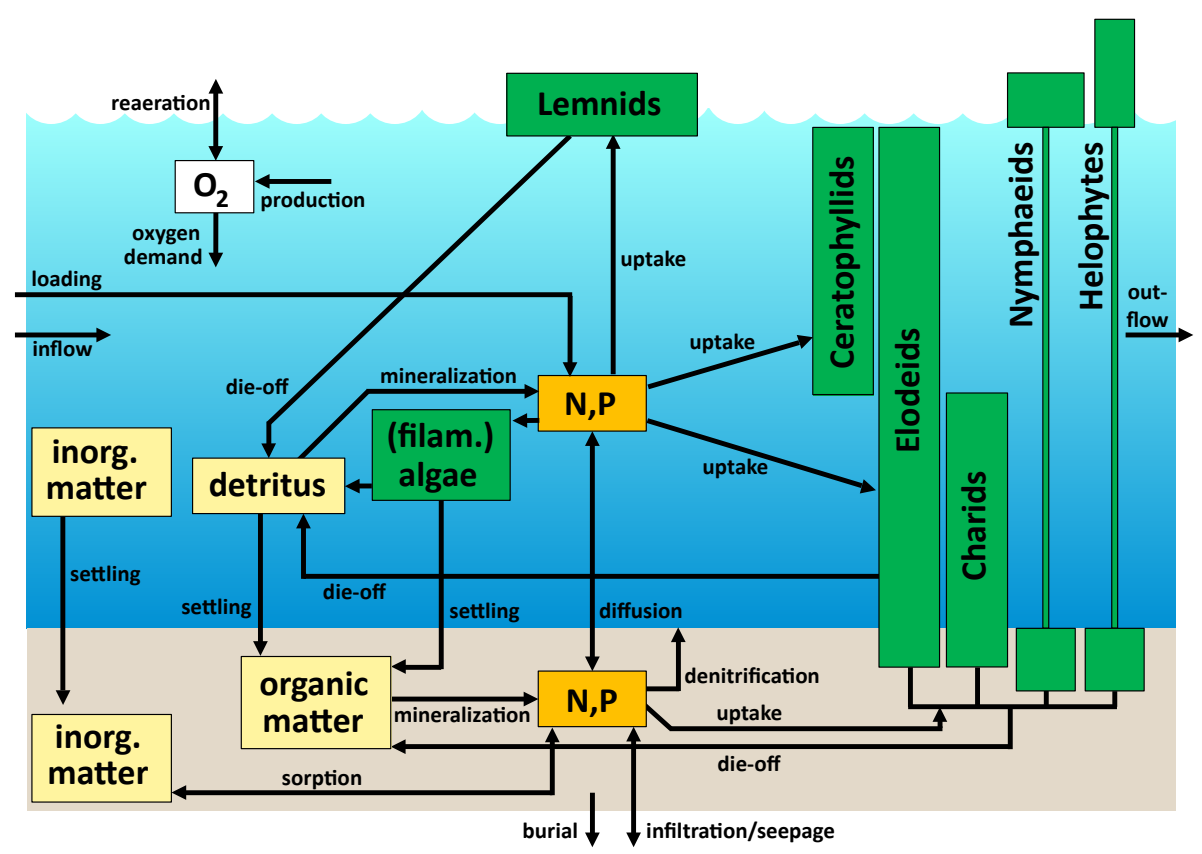

Figure 1.5. Components and processes of the ecosystem model PCDitch, after Janse (1998).

Over the years, PCDitch proved to be helpful in understanding, predicting and restoring the ecology of ditches, and therefore obtained an increasingly prominent role in water management. A key feature contributing to this is that PCDitch can predict the critical nutrient loading at which a ditch switches 
from submerged-plant dominance to free-floating plants, and backwards. Such critical nutrient loading provides managers with a clear target that can easily be communicated. This resulted in the application of PCDitch to different polders systems in the Netherlands (e.g. Witteveen + Bos 2010b). The critical nutrient loading was found to depend on ditch characteristics, like depth, sediment composition and water flow (Van Liere et al. 2007). PCDitch predicts no alternative stable states, at least for the settings of the calibrated model that resemble an average Dutch ditch (Janse 1998). Apart from a preliminary study by Van Liere et al. (2002), the behaviour of PCDitch has not yet been systematically explored in a spatial context (network of interconnected ditches).

\subsection{General aim and thesis outline}

The main objective of this thesis is to develop concepts that enable us to better understand, predict and restore the ecology of ditches. For this I combined models of different complexity, as illustrated by Fig. 1.6 that visualizes the thesis outline. I will now explain the research objectives per chapter.

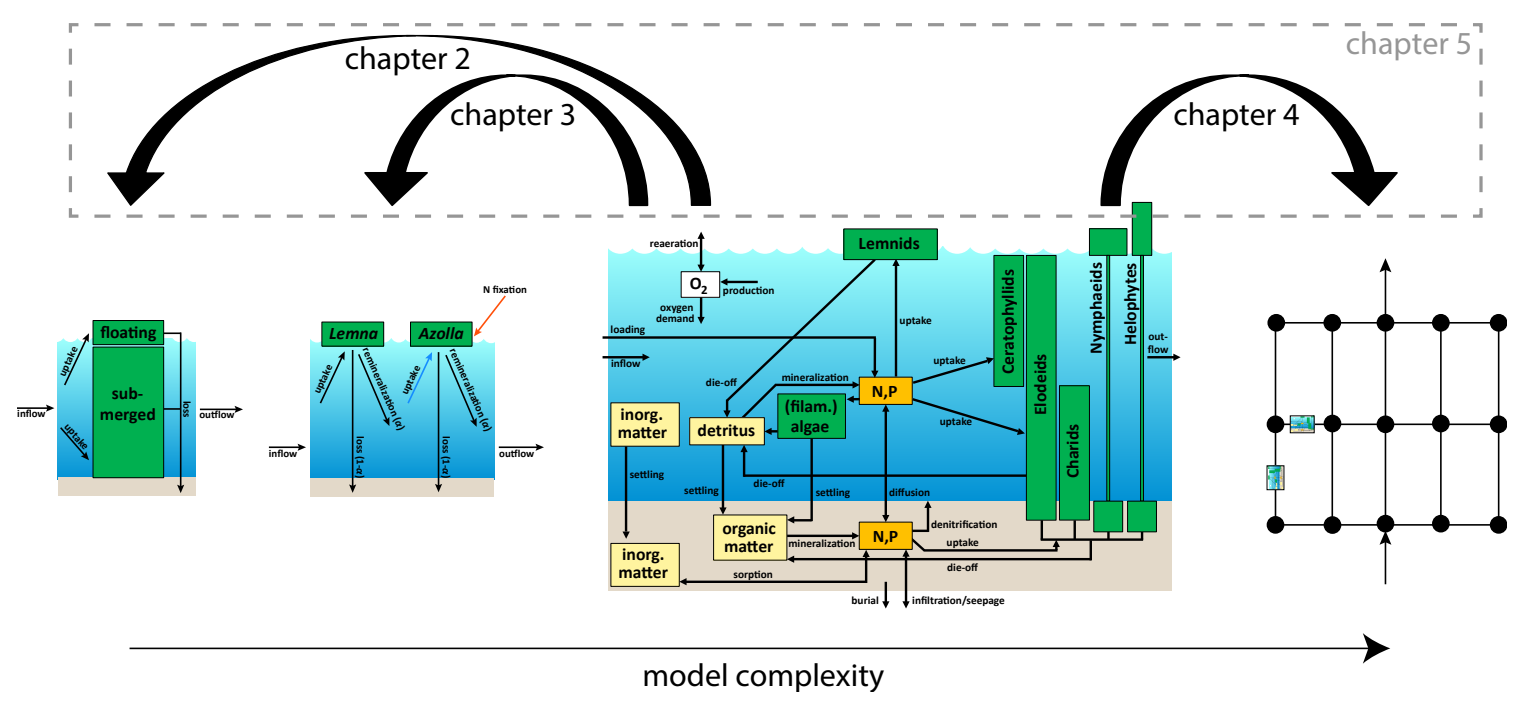

Figure 1.6. Schematic outline of this thesis, illustrating the use of models with different complexity, with the complex ecosystem model PCDitch as a starting point. Chapter 5 presents an approach which facilitates the up- and downscaling in model complexity as depicted by the arrows. 


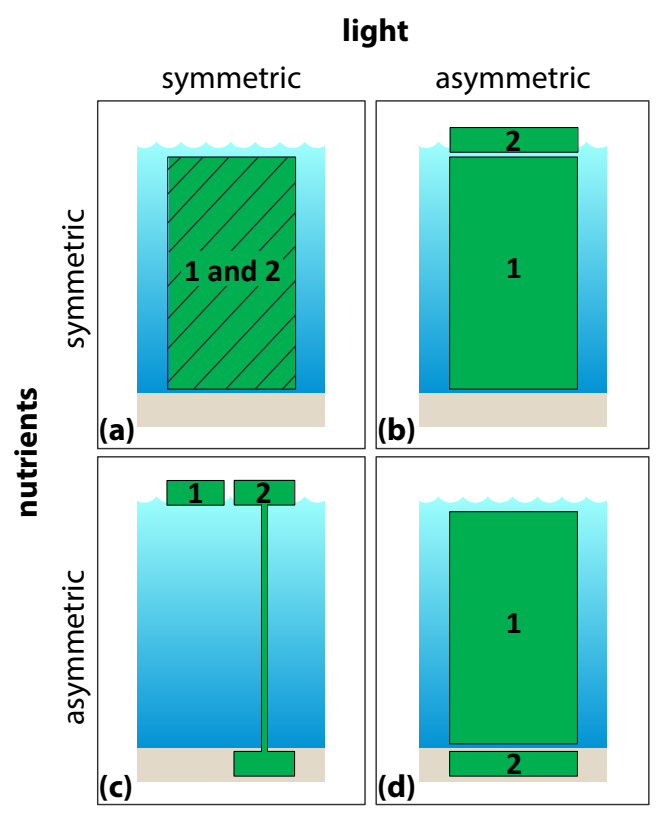

Figure 1.7. Potential configurations for two primary producers competing for light and nutrients in a well-mixed water column, having equal access to nutrients or light — implying symmetric competition — or differential access leading to asymmetric competition.

\section{Extend competition theory to explore whether ditches have alternative sta- ble states (chapter 2)}

It is yet unknown for ditches if submerged-plant dominance and floatingplant dominance can be considered as alternative stable states. This can be better understood by regarding the competition between submerged and free-floating plants for nutrients and light. However, there is no mechanistic theory yet that addresses this competition. Huisman and Weissing (1995) described the competition of submerged (algal) species having equal access to nutrients and light (Box 1.1, Fig. 1.7a), and Jäger and Diehl (2014) assessed the competition of benthic and pelagic algae with differential access to light and nutrients (Fig. 1.7d), but theory on layered communities of water plants is still lacking (Fig. 1.7b). To fill this knowledge gap, I construct a simple model of this layered configuration. This provides an essential step to better understand under which environmental conditions floating plants dominate and to explore - in combination with field data - if submerged versus floating-plant dominance are likely to be alternative stable states. 


\section{Explore whether eutrophic ditches can be restored by reducing $\mathbf{N}$ inputs (chapter 3)}

There is an ongoing debate among ecologists about the role of $\mathrm{N}$ and $\mathrm{P}$ in the restoration of eutrophic aquatic ecosystems. Main point of debate is whether nutrient reduction strategies should solely focus on $\mathrm{P}$ or also on $\mathrm{N}$ ? It has been argued that reducing $\mathrm{N}$ inputs is ineffective, because it favors species that can fix $\mathrm{N}_{2}$ from the atmosphere and that eventually provide the system with enough $\mathrm{N}$ to correct for $\mathrm{N}$ deficits, and thus perpetuate $\mathrm{P}$-limitation (Schindler et al. 2008). Others argue that $\mathrm{N}_{2}$-fixing species cannot meet the $\mathrm{N}$ requirements of the eutrophic ecosystem due to constraints on $\mathrm{N}_{2}$-fixation (Lewis and Wurtsbaugh 2008), implying that steering on $\mathrm{N}$ can be effective. This debate mainly focusses on lakes. But what about ditches, can floating-plant dominance be combatted by reducing $\mathrm{N}$ inputs or does it lead to the rise of the free-floating $\mathrm{N}_{2}$-fixing water fern Azolla? And is Azolla able to correct for $\mathrm{N}$ deficits and perpetuate P-limitation? To answer these questions I construct a simple model and a complex PCDitch-based model to regard the competition of Azolla with the non- $\mathrm{N}_{2}$-fixing duckweed (Lemna) for $\mathrm{N}, \mathrm{P}$ and light. In combination with field data, this study adds new fuel to the debate on how to restore eutrophic ecosystems.

Investigate whether each ditch in a network is equally vulnerable to floating plant-dominance (chapter 4)

Dutch polder systems are characterized by dense networks of connected ditches. The water flow in these ditches typically increases in the direction of the polder outlet, as each ditch receives water (and nutrients) from the adjacent land by lateral runoff, which accumulates in downstream direction. I explore if this increase of water flow, and associated increase of nutrient loading as the water carries nutrients and to a less extent also biota, affects the vulnerability of a ditch to floating-plant dominance. Are some ditches more vulnerable than others depending on their distance to the polder outlet? To explore this, I first construct a simple model to analyze how the increase of water flow affects the nutrient concentration in a chain of ditches, and then look into the consequences for ecology by applying PCDitch to the same chain of ditches, as well as to a network of ditches for which I coupled PCDitch to the hydrodynamic SOBEK model. This study provides a new basic concept on how hydrology affects ecology. 
Explore a method that eases the development, analysis and implementation of models (chapter 5)

Modelling comes with a technical cost. First, the model equations have to be implemented in a programming language. Next, to run the model, the equations need to be compiled or interpreted by a computer in order to numerically solve them. There are dozens of modelling environments in which this can be done (Janssen et al. 2015), all having their own specific language and framework requirements. As a result, ecological models are often locked in a certain modelling framework, which fragments the field of modelling. In addition, the model code is often polluted by technical implementation code, distracting from the ecology behind it. To overcome these issues, we developed a 'Database Approach To Modelling' (DATM; Mooij et al. 2014), in which a model is stored in a clear and framework-independent way, from which it can be easily translated to a modelling environment of choice. I describe the opportunities that this approach creates, and illustrate this by implementing PCDitch and PCLake (Janse 2005) in different frameworks. Furthermore, I use DATM throughout this thesis for developing models of different complexity, as the database format turned out to be very useful for that. 



\section{Chapter 2}

\section{Competition for light and nutrients in layered communities of aquatic plants}

van Gerven, L. P. A., J. J. M. de Klein, D. J. Gerla, B. W. Kooi, J. J. Kuiper, and W. M. Mooij. 2015. Competition for Light and Nutrients in Layered Communities of Aquatic Plants. The American Naturalist 186: 72-83.

Abstract. Dominance of free-floating plants poses a threat to the biodiversity in many freshwater ecosystems. Here, we propose a theoretical framework to understand this dominance, by modeling the competition for light and nutrients in a layered community of floating and submerged plants. The model shows that at high supply of light and nutrients, floating plants always dominate due to their primacy for light, even when submerged plants have lower minimal resource requirements. The model also shows that floating-plant dominance cannot be an alternative stable state in light-limited environments but only in nutrient-limited environments, depending on the plants'resource consumption traits. Compared to unlayered communities, the asymmetry in competition for light - while maintaining symmetry in competition for nutrients - leads to fundamentally different results: competition outcomes can no longer be predicted from species traits like minimal resource requirements ( $R^{*}$ rule) and resource consumption. Also, the same two species can, depending on the environment, coexist or show alternative stable states. When applied to two common plant species in temperate regions, both the model and field data suggest that floating-plant dominance is unlikely to be an alternative stable state. 


\subsection{Introduction}

Dense mats of free-floating plants are a threat to the biodiversity and ecological functioning of aquatic ecosystems, ranging from tropical lakes (Brendonck et al. 2003) to temperate ditches and ponds (Janse and van Puijenbroek 1998). Underneath these mats, the water often becomes too dark for submerged plants to photosynthesize (Morris et al. 2004) and too low in dissolved oxygen for macrofauna and fish to survive (Portielje and Roijackers 1995). It is therefore crucial to know what drives the occurrence of these dense mats of floating plants and if they are a self-enhancing (alternative) stable state, as suggested by Scheffer et al. (2003).

The in-water nutrient availability is of vital importance for the development of free-floating plants and is affected by submerged plants (Madsen and Cedergreen 2002). Therefore, the occurrence of free-floating plants can be understood by studying their competition with submerged plants for light and nutrients. Although there have been empirical studies on the competition between floating and submerged plants using laboratory experiments (Janes et al. 1996; Szabo et al. 2010), mesocosm experiments (Feuchtmayr et al. 2009; Lu et al. 2013; Netten et al. 2010; Smith 2014) and field experiments (Forchhammer 1999; Portielje and Roijackers 1995), the theoretical understanding of the competition between floating and submerged plants for light and nutrients is still limited.

Most mechanistic theory on competition for light and nutrients is developed for phytoplankton. For example, $I_{\text {out }}^{*}-R^{*}$ theory describes the competition of phytoplankton species for light and nutrients in a mixed water column (Huisman and Weissing 1995). It is an extension of $R^{*}$ theory, which describes competition of species for nutrients (Tilman 1982). An elegant feature of $I_{\text {out }}^{*}-R^{*}$ theory (and also $R^{*}$ theory) is that one can predict the competition outcome from species traits, like minimal resource requirements ( $R^{*}$ rule) and resource consumption. Yet, this framework considers the competition between species that have the same position in the water column, while floating and submerged plants have different positions. The (vertical) position of a species matters when considering light, because light forms a gradient with depth, due to light attenuation by biomass and background extinction (Kirk 1994). In a vertically layered community the competition for light therefore becomes asymmetric: the upper species (the floating plant) has the primacy for light and shades the lower species (the submerged plant) and therefore has a competitive advantage. The competition for nutrients on the other hand is 
not asymmetric as both floating and submerged plants compete for nutrients in the same medium (water column).

Mechanistic resource competition theory has been extended to include asymmetrical competition. Yet, none of the available theoretical frameworks are suited to describe the competition between floating and submerged plants. Huisman et al. (1999a), Weissing and Huisman (1994) and Perry et al. (2003) considered asymmetrical competition for light, but did not take competition for nutrients into account. In cases where nutrients were explicitly modelled, either the mechanism underlying the layeredness differed or the competition was not only asymmetric for light but also for nutrients (double asymmetry).

For example, in pelagic phytoplankton communities the layeredness emerges from limited vertical mixing of the water column (Ryabov 2012; Ryabov and Blasius 2011; Yoshiyama et al. 2009) and from extra movement of phytoplankton related to sinking or buoyancy regulation (Huisman et al. 2006; Ryabov et al. 2010), with vertical nutrient gradients as a result. For floating and submerged plants, however, the layeredness does not emerge - as they do not wander through the water column - but is directly imposed by their growth form. The model of Jäger and Diehl (2014) gives an example of double asymmetry: not only do the upper species (pelagic algae) have a primacy for light (similar to floating plants), but also do the lower species (benthic algae) have a primacy for nutrients, supplied from below, whereas floating and submerged plants directly compete for the same nutrients. Interestingly, these more complex examples of asymmetrical competition show that the competition outcome cannot simply be predicted anymore from species traits alone.

The only model that does describe the competition between floating and submerged plants is the model of Scheffer et al. (2003). However, this model is not fully mechanistic - for example it does not have a closed nutrient and light balance - and is not embedded in classical resource competition theory. This motivated us to develop a model that does comply with these aspects.

Here we present a mechanistic model that describes the competition for light and nutrients in a layered community of floating and submerged plants. We hypothesize that the floating plant, due to its primacy for light, can outcompete the submerged plant, even when the submerged plant has lower resource requirements than the floating plant. This implies that the most parsimonious form of asymmetry in competition - being asymmetry in competition for light while maintaining symmetry in competition for nutrients in a homogeneous nutrient environment - is sufficient to make that the com- 
petition outcome cannot be predicted anymore from species traits alone, and hence, that environmental conditions co-determine the competition outcome.

To test this and to better understand when floating plants dominate, we analyzed our model and its competition outcome for a wide range of species traits and environmental conditions, focusing on the requirements for single species dominance, coexistence or alternative stable states. Furthermore we analyzed the competition outcome for floating and submerged plant species that are common in temperate regions and discuss the likelihood of floatingplant dominance to be an alternative stable state.

\subsection{Methods}

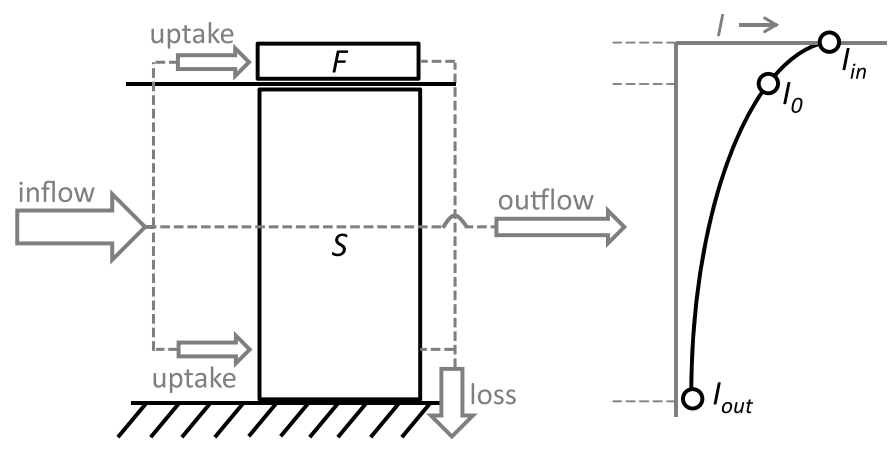

Figure 2.1. The competition model of floating plant $F$ and submerged plant $S$ in a vertical water column with nutrient fluxes (left) and light intensity (right).

\section{Competition model}

Our model describes competition for light and nutrients in a layered community of floating plants $(F)$ and submerged plants $(S)$ in a vertical water column (Fig. 2.1, Table 2.1). The model extends $I_{\text {out }}^{*}-R^{*}$ theory (Huisman and Weissing 1995) - describing the symmetric competition for nutrients and light of species having the same position - for species with a different fixed vertical position, resulting in asymmetrical competition for light. It consists of three differential equations, describing the dynamics of floating and submerged plant biomass (in $\mathrm{gDW} \mathrm{m}^{-2}$ ) and nutrients (in $\mathrm{gN} \mathrm{m}^{-3}$ ). The plant biomass dynamics depend on the balance between loss rate $m$ and growth rate $p$ :

$$
\frac{\mathrm{d} F}{\mathrm{~d} t}=\left(p_{F}-m_{F}\right) F
$$


Table 2.1. Model symbols and their definition.

\begin{tabular}{|c|c|c|c|c|}
\hline Symbol & Description & Unit & $\begin{array}{l}\text { Default value } \\
\text { (for } F \text { and } S \text { ) }\end{array}$ & $\begin{array}{c}\text { Common plants } \\
\text { (Lemna sp., Elodea sp.) }^{a}\end{array}$ \\
\hline \multicolumn{5}{|c|}{ State variables: } \\
\hline$F$ & Floating plant biomass & $\mathrm{gDW} \mathrm{m}^{-2}$ & & \\
\hline$S$ & Submerged plant biomass & gDW m ${ }^{-2}$ & & \\
\hline$R$ & Nutrient concentration in water column & $\mathrm{gN} \mathrm{m}^{-3}$ & & \\
\hline \multicolumn{5}{|c|}{ Light functions: } \\
\hline$I$ & Light intensity at depth & $\mathrm{J} \mathrm{m}^{-2} \mathrm{~s}^{-1}$ & & \\
\hline$I_{0}(F)$ & Light intensity at top of water column & $\mathrm{J} \mathrm{m}^{-2} \mathrm{~s}^{-1}$ & & \\
\hline$I_{\text {out }}(S, F)$ & Light intensity at bottom of water column & $\mathrm{J} \mathrm{m}^{-2} \mathrm{~s}^{-1}$ & & \\
\hline \multicolumn{5}{|c|}{ Plant species parameters and functions: } \\
\hline$p_{i}(I, R)$ & Growth rate of plant $i$ & day $^{-1}$ & & \\
\hline$f_{R, i}(R)$ & Nutrient limitation factor of growth & - & & \\
\hline$f_{I, i}(I)$ & Light limitation factor of growth & - & & \\
\hline$p_{\max , i}$ & Maximum growth rate & day $^{-1}$ & .3 & $.4, .32$ \\
\hline$m_{i}$ & Loss rate & day $^{-1}$ & .05 & $.05, .029$ \\
\hline$k_{i}$ & Light attenuation coefficient & $\mathrm{m}^{2} \mathrm{gDW}^{-1}$ & .03 & $.03, .03$ \\
\hline$H_{i}$ & Half-saturation constant for light & $\mathrm{J} \mathrm{m}^{-2} \mathrm{~s}^{-1}$ & 30 & 32,32 \\
\hline \multirow[t]{2}{*}{$M_{i}$} & Half-saturation constant for nutrients & $\mathrm{gN} \mathrm{m}^{-3}$ & .5 & $2.5, .5$ \\
\hline & & $\mathrm{gP} \mathrm{m}^{-3}$ & & $.25, .05$ \\
\hline \multirow[t]{2}{*}{$c_{i}$} & Nutrient to dry weight ratio & $\mathrm{gN} \mathrm{gDW}^{-1}$ & .03 & $.07, .0225$ \\
\hline & & $\mathrm{gP} \mathrm{gDW}^{-1}$ & & $.015, .00215$ \\
\hline \multicolumn{5}{|c|}{ Environmental parameters: } \\
\hline$r_{i n}$ & Nutrient loading & $\mathrm{gN} \mathrm{m}^{-2}$ day $^{-1}$ & $0-.5$ & \\
\hline$I_{\text {in }}$ & Incoming light intensity & $\mathrm{J} \mathrm{m}^{-2} \mathrm{~s}^{-1}$ & $0-1,000$ & \\
\hline$z_{B}$ & Water column depth & $\mathrm{m}$ & $.1-1$ & \\
\hline$D$ & Dilution rate of water column & day $^{-1}$ & $.01-.5$ & \\
\hline$K_{b g}$ & Background light attenuation & $\mathrm{m}^{-1}$ & $0-5$ & \\
\hline
\end{tabular}

Note: DW= dry weight

${ }^{a}$ From Janse 1998.

$$
\frac{\mathrm{d} S}{\mathrm{~d} t}=\left(p_{S}-m_{S}\right) S .
$$

The growth rate $p(I, R)$ is co-limited by nutrient concentration $R$ and light intensity $I$ :

$$
p_{i}=f_{R, i} f_{I, i} p_{\max , i},
$$

where $i$ stands for $S$ or $F, p_{\max }$ is the maximum growth rate, $f_{R}(R)$ is the nutrient limitation factor and $f_{I}(I)$ is the light limitation factor. Nutrient limitation is characterized by a Michaelis-Menten function with half-saturation constant $M$ :

$$
f_{R, i}=\frac{R}{R+M_{i}} .
$$

Light limitation is also characterized by a Michaelis-Menten function, with half-saturation constant $H$. Since light forms a gradient with plant biomass 
and therefore varies with depth (Kirk 1994), the average light limitation is obtained by integrating over the depth covered by the plant, here expressed in terms of $I$, the light intensity at depth:

$$
\begin{gathered}
f_{I, F}=\frac{1}{k_{F} F} \int_{I_{0}}^{I_{i n}} \frac{1}{H_{F}+I} \mathrm{~d} I=\frac{1}{k_{F} F} \ln \left(\frac{H_{F}+I_{\text {in }}}{H_{F}+I_{0}}\right) \\
f_{I, S}=\frac{1}{k_{S} S+K_{b g} z_{B}} \int_{I_{\text {out }}}^{I_{0}} \frac{1}{H_{S}+I} \mathrm{~d} I=\frac{1}{k_{S} S+K_{\text {bg }} z_{B}} \ln \left(\frac{H_{S}+I_{0}}{H_{S}+I_{\text {out }}}\right),
\end{gathered}
$$

where in solving the integral, the plant biomass is assumed to be uniformly distributed with depth. For details on the derivation of the light limitation factor, see Huisman and Weissing (1994). $I_{i n}$ is the incoming light intensity, $I_{0}$ is the light intensity below the floating plants and above the submerged plants, and $I_{\text {out }}$ is the light intensity below the submerged plants (Fig. 1). $I_{0}$ and $I_{\text {out }}$ depend on the light attenuation by plant biomass according to Lambert-Beer's law:

$$
\begin{gathered}
I_{0}=I_{\text {in }} e^{-k_{F} F} \\
I_{\text {out }}=I_{0} e^{-\left(k_{S} S+K_{\text {bg }} z_{B}\right)},
\end{gathered}
$$

where $k$ is the light attenuation coefficient of the plant. $I_{\text {out }}$ also depends on the background light attenuation $K_{b g}$ of the water and the water column depth $z_{B}$. The nutrient concentration $R$ in the water column is assumed to be homogeneous over depth, which is a reasonable assumption for shallow waters. Nutrient dynamics depend on the nutrient consumption by the plants and on the input and output of nutrients related to water flow $q$ :

$$
\frac{\mathrm{d} R}{\mathrm{~d} t}=\frac{q}{z_{B}}\left(R_{i n}-R\right)-\frac{1}{z_{B}}\left(c_{F} p_{F} F+c_{S} p_{S} S\right)=\frac{r_{i n}}{z_{B}}-D R-\frac{1}{z_{B}}\left(c_{F} p_{F} F+c_{S} p_{S} S\right),
$$

where $r_{i n}$ is the nutrient loading which can be converted to a nutrient concentration $R_{i n}$ of the inflowing water $\left(R_{i n}=r_{i n} / q\right), D$ is the dilution rate of the water column $\left(D=q / z_{B}\right)$ and the nutrient consumption by water plants equals the biomass growth multiplied by $c$, the nutrient content per unit of biomass. Division by $z_{B}$ gives the nutrient consumption per unit volume.

\section{Minimal resource requirements and resource consumption of the plants}

In general resource competition theory, such as $R^{*}$ theory (Tilman 1980; Tilman 1982) and $I_{\text {out }}^{*}-R^{*}$ theory (Huisman and Weissing 1995), the species' minimal resource requirements and resource consumption are important 
traits to predict the competition outcome. The minimal resource requirements are the lowest level of light and nutrients at which the plant can persist. The resource consumption is related to the consumption vector, which represents the proportion of resources consumed when the species is at equilibrium (Tilman 1980). We derived these traits from the model equations (see Appendix A) to analyse their importance when the competition is asymmetric for light.

\section{Model analysis}

To test how asymmetry in light competition determines the competition outcome between submerged and floating plant species, we analyzed our model for a wide range of species traits and environmental conditions. We assumed nitrogen $(\mathrm{N})$ to be the limiting nutrient. However, we could also have chosen phosphorus (P), because P-limitation seems to be equally widespread for primary producers in freshwater ecosystems (Elser et al. 2007). We have checked by model simulations (not shown here) that the choice for $\mathrm{N}$ or $\mathrm{P}$ as a limiting nutrient does not affect the competition outcome qualitatively.

We performed the model analysis in several steps. We started our analysis by evaluating the competition outcome when both plants have equal traits (Fig. 2.2A), by using the same default parameter values for each plant (table 1). These values were in the middle of ranges reported in literature for common floating and submerged plants (Janse 1998; Janse 2005; Scheffer et al. 2003). We plotted the competition outcome in the $r_{i n}-I_{i n}$ plane, i.e. for various combinations of light supply $I_{i n}$ and nutrient loading $r_{i n}$, mimicking a wide range of environmental conditions.

Secondly, we analysed the effect of different minimal resource requirements of the plants on the competition outcome in the $r_{i n}-I_{i n}$ plane. This was done by varying the half-saturation constants for nutrient limitation $(M)$ and light limitation $(H)$ (see Eq. A.1 and A.2 in Appendix A) such that the submerged plant has lower minimal requirements for light (Fig. 2.2B), nutrients (Fig. 2.2C) or light and nutrients (Fig. 2.2D). As it is often assumed that submerged plants have lower minimal resource requirements than floating plants, we continued our study focussing on this configuration. We aimed at obtaining a more thorough understanding of the competition outcome of this configuration by analysing the equilibrium biomass of both plants along a gradient of light supply and nutrient loading (Fig. 2.3). We zoomed in on these gradients that captured the most complex and interesting model outcomes. 
Next, we were interested in the effect of the plant's resource consumption traits on the competition outcome in the $r_{i n}-I_{i n}$ plane (Fig. 2.4). Therefore, we varied the plant's ratio of light attenuation $k$ to nutrient content $c$ as this ratio controls the resource consumption (see equations A.6 and A.7 in Appendix A). Also we analysed how the competition outcome depends on environmental conditions other than light and nutrient supply, such as water column depth $z_{B}$, dilution rate $D$ and water background light attenuation $K_{b g}$ (Fig. 2.5), by varying them within the ranges reported in table 1.

We concluded our analyses by evaluating the competition outcome for two floating and submerged plant species that are common in temperate regions, respectively Lemna sp. (duckweed) and Elodea sp. (waterweed) (Fig. 2.6). We did this for both $\mathrm{N}$ and $\mathrm{P}$ as a limiting nutrient. We retrieved the parameter values (table 1) from the established ecosystem model PCDitch (Janse 1998), which simulates the competition between water plants with different growth forms. PCDitch provides a coherent set of vegetation parameters, derived from literature and calibrated on experimental Dutch ditches. For Lemna sp. the light parameters $H$ and $k$ are not defined in PCDitch, because its light limitation is incorporated differently in PCDitch compared to our model. We assumed the half-saturation constant for light limitation $H$ to be the same for Lemna sp. and Elodea sp. The light attenuation coefficient $k$ was for both plants chosen within realistic ranges (Krause-Jensen and Sand-Jensen 1998) such that the maximum plant biomasses are realistic. Furthermore, for the nutrient content $c$ of the plants, we used the average of the minimum and maximum content provided by PCDitch.

\section{Analysis methods}

We solved for the competition outcome numerically, since we did not find any simple expressions for the plants' equilibrium densities. We used the software packages AUTO (Doedel and Oldeman 2009) and R (R Core Team 2013) for numerical analysis and to determine the stability of the equilibria and the position of bifurcations in the $r_{i n}-I_{i n}$ plane.

Supplementary to bifurcation analysis, we performed numerical invasion analysis to determine the competition outcome, by calculating whether a species (the invader) can invade an equilibrium density of another species (the resident) (Chesson 2000). In a two-species community like ours, the species stably coexist when each species can invade (+) an equilibrium density of the other $(++)$, the species show alternative stable states when neither can invade $(-)$ an equilibrium density of the other $(--)$, while $(+-)$ or $(-+)$ leads 
to competitive exclusion of the species that cannot invade (e.g. Gerla et al. 2011). In the case of alternative stable states, the species that establishes first outcompetes the other.

\subsection{Results}

\section{The importance of species traits}

When both plants have equal traits, the floating plant outcompetes the submerged plant for all combinations of light supply and nutrient loading (Fig. 2.2A), provided that the resource supply is high enough for the plants to persist.

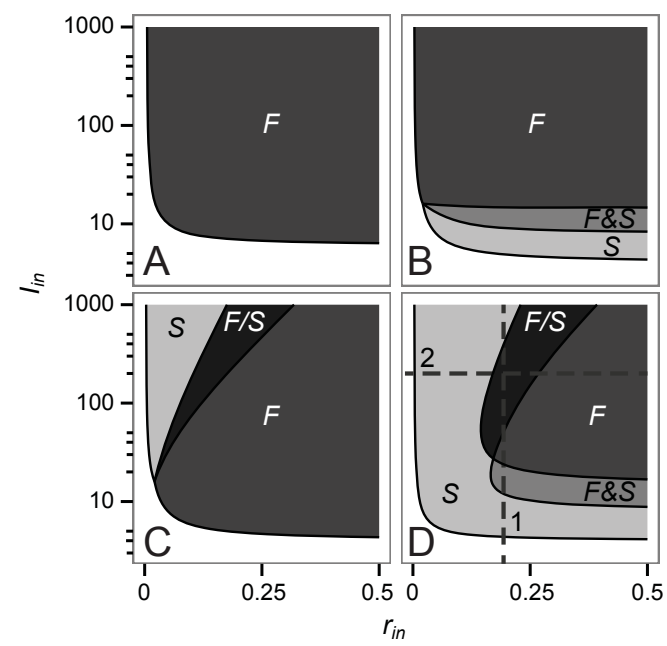

Figure 2.2. Outcome of the competition of floating plant $F$ and submerged plant $S$ for combinations of light supply $I_{i n}\left(\mathrm{~W} \mathrm{~m}^{-2}\right)$ and nutrient loading $r_{i n}\left(\mathrm{gN} \mathrm{m}^{-2} \mathrm{day}^{-1}\right)$. ' $F$ ' indicates regions where the floating plant persists, in region ' $S$ ' the submerged plant persists, in ' $F \& S$ ' the plants coexist and in ' $F / S$ ' the plants are alternative stable states. Note that at low resource supply both plants cannot persist. In panel A the plants have equal traits, whereas in panels $\mathrm{B}, \mathrm{C}$ and $\mathrm{D}$ they have different resource requirements: the submerged plant has the lowest nutrient requirements in panels $C$ and $D$, and has the lowest light requirements in panels $B$ and $D$. The lines 1 and 2 in panel $D$ are transects used for the bifurcation analysis in Fig. 2.3. Environmental parameters: $K_{b g}=0, z_{B}=0.5, D=0.1$. The plant parameters equal the default values given in table 1, except for $M$ and $H$ that determine the minimal resource requirements: panel $\mathrm{A} ; M_{F}=M_{S}=0.5, H_{F}=H_{S}=30$, panel B; $M_{F}=0.3, M_{S}=0.7, H_{F}=40, H_{S}=20$, panel C; $M_{F}=0.7, M_{S}=0.3, H_{F}=20, H_{S}=40$, panel D; $M_{F}=0.7, M_{S}=0.3, H_{F}=40, H_{S}=$ 20 . 
This illustrates the asymmetry in light competition in layered communities and the resulting competitive advantage of the floating plant: it has the primacy for light, shades the submerged plant and does not compete for light with the submerged plant.

The submerged plant can only outcompete the floating plant when it has lower minimal resource requirements for light (Fig. 2.2B), nutrients (Fig. 2.2C) or light and nutrients (Fig. 2.2D), provided that the supply of the resource for which it has the lowest requirements is low enough. At higher resource supply, a submerged plant with lower resource requirements coexists or shows alternative states with - or is even outcompeted by - the floating plant. At high enough supply of light and nutrients, the floating plant always outcompetes the submerged plant. For further analysis, we used the configuration where the submerged plant has the lowest requirements for both light and nutrients (Fig. 2.2D) as a starting point, as this configuration is assumed to be the most common one, and gives the richest competition outcome.

To better understand the competition outcome, we analysed the equilibrium biomass of both plants along a gradient of light supply (transect 1 in Fig. 2.2D, results plotted in Fig. 2.3A). The submerged plant can persist at a lower light supply (bifurcation 1), since it has the lowest light requirements. When the light supply is increased, there is enough light for the floating plant to persist (bifurcation 2) and the plants coexist, until the floating plant reaches a critical biomass and intercepts too much light for the submerged plant to persist (bifurcation 3). With further increasing light supply, nutrient limitation takes precedence over light limitation. From bifurcation 4 onwards they show alternative states since both plants cannot invade the equilibrium density of the other: the submerged plant reduces the nutrient concentration to levels too low for the floating plant to invade and the floating plant intercepts too much light for the submerged plant to invade. At even higher light supply (above that of bifurcation 5), only the submerged plant persists because the floating plant no longer intercepts enough light to prevent the submerged plant from invading.

In a similar manner, we analysed the competition outcome by regarding the equilibrium biomass along a gradient of nutrient loading (transect 2 in Fig. 2.2D, results plotted in Fig. 2.3B). The submerged plant persists at a lower nutrient loading (bifurcation 1), as it has the lowest nutrient requirements. With increasing nutrient loading (up to bifurcation 2), even if the floating plant is able to persist when alone, it is outcompeted by the submerged plant. From bifurcation 2 onwards they show alternative states since both plants cannot 

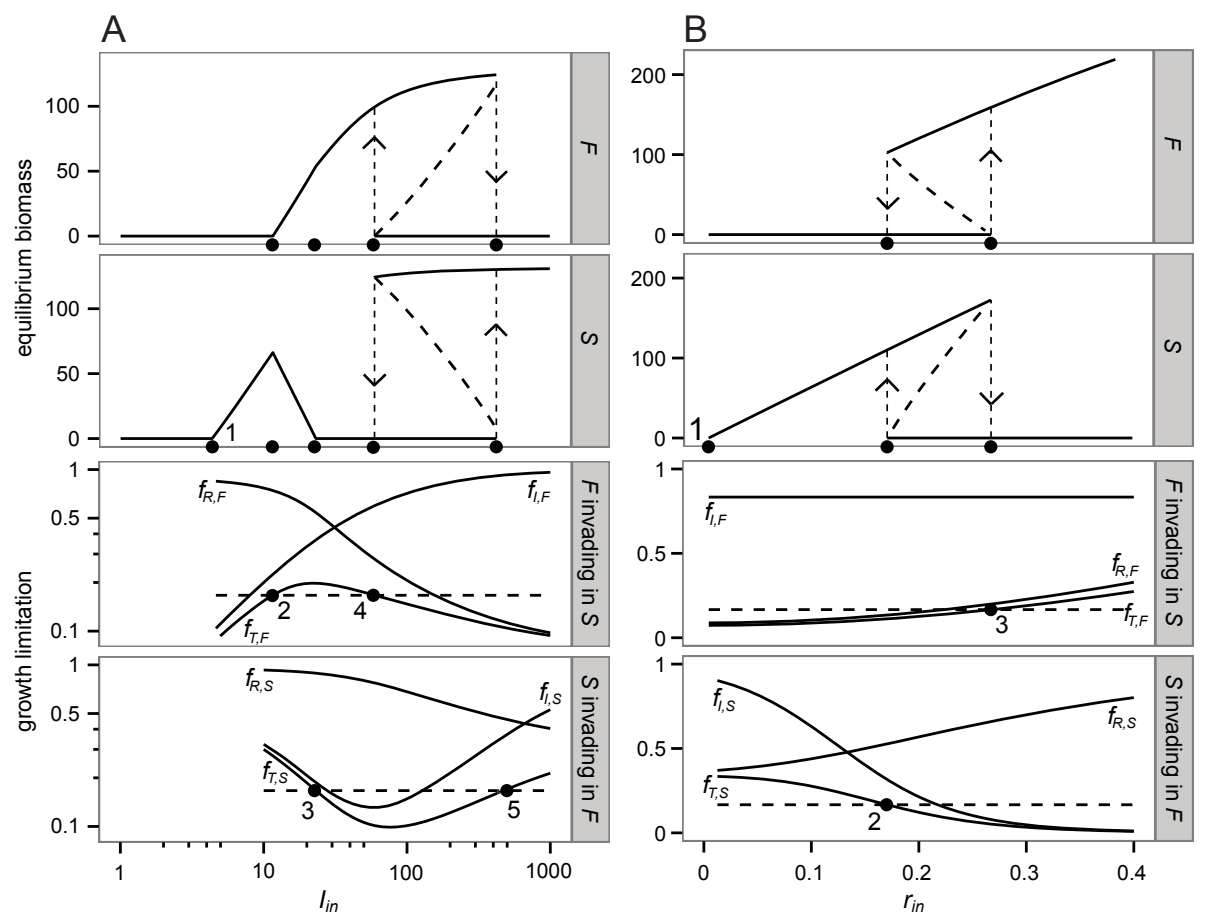

Figure 2.3. Effect of (A) light supply $I_{i n}\left(\mathrm{~W} \mathrm{~m}^{-2}\right)$ and (B) nutrient loading $r_{i n}$ (gN $\mathrm{m}^{-2}$ day $^{-1}$ ) on equilibrium biomass $\left(\mathrm{gDW} \mathrm{m}{ }^{-2}\right.$ ) of floating plant $F$ and submerged plant $S$ (upper panels) and on the growth limitation of the plant when invading an equilibrium density of the other (lower panels). Catastrophic shifts are indicated with arrows and the dashed lines (upper panels) give the unstable coexistence equilibrium. The dots denote transcritical bifurcations. Bifurcations 2 to 5 are the invasion boundaries where the total growth limitation $f_{T}$ of the invader $\left(f_{T}=f_{R} f_{I}\right)$ equals the growth limitation level at which the invader can persist (dashed line). Note that the growth limitation is maximum at $f_{T}=0$ and is absent at $f_{T}=1$. The parameters values are the same as in Fig. 2.2D with $r_{i n}=0.2$ in panel A (see transect 1 in Fig. 2.2D) and $I_{i n}=200$ in panel B (see transect 2 in Fig. 2.2D). 
invade the equilibrium density of the other: the floating plant intercepts too much light for the submerged plant to invade and the submerged plant keeps the nutrient concentration too low for the floating plant to invade. At higher nutrient supply (from bifurcation 3 onwards), only the floating plant persists as the submerged plant cannot keep the nutrient concentration low enough to prevent the floating plant from invading.

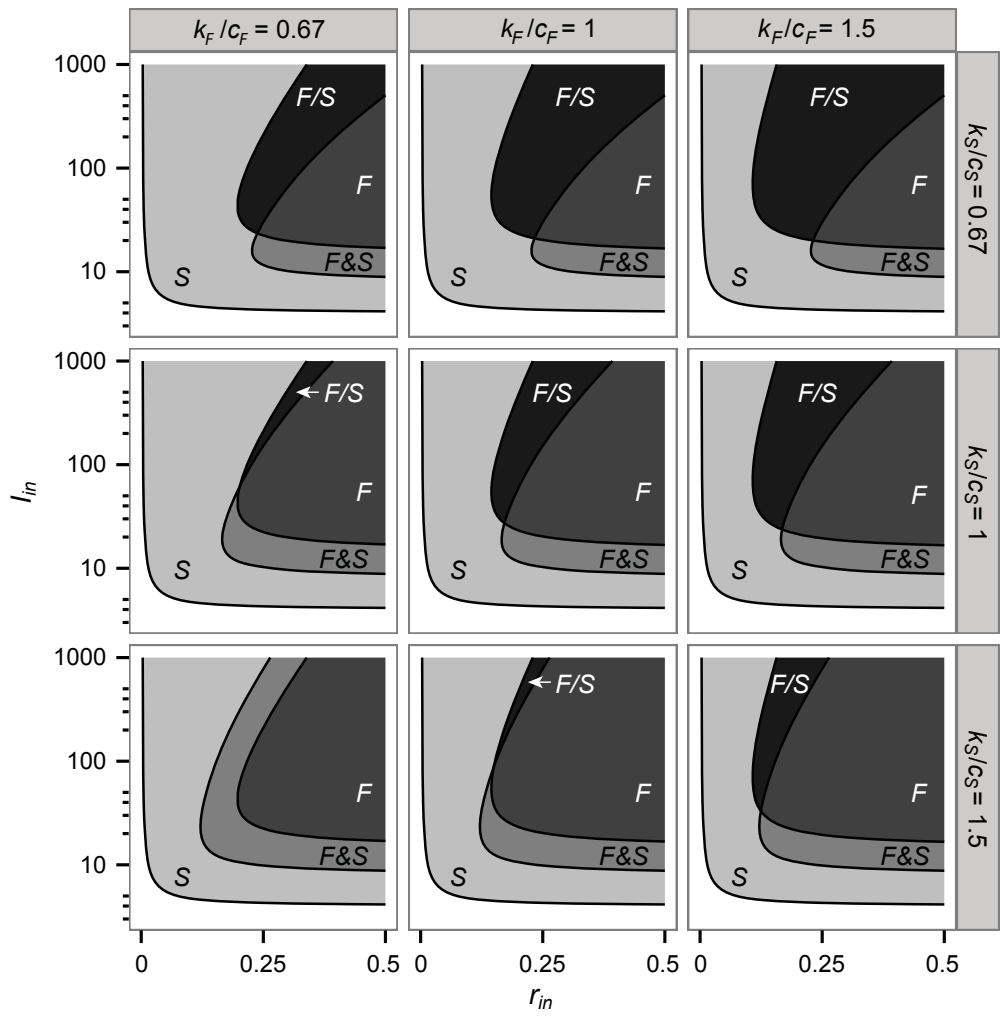

Figure 2.4. Outcome of the competition of floating plant $F$ and submerged plant $S$ when the plants have different resource consumption traits, resulting from different ratios of light attenuation coefficient $k$ over nutrient to dry weight ratio $c$. Except for $k$ and $c$, the parameter values are the same as in Fig. 2.2D (which equals the center graph).

When changing the resource consumption traits of both plants - by changing the ratio of light attenuation coefficient $k$ over nutrient content $c$ - the competition outcome is affected mainly in 'nutrient-limited environments' (Fig. 2.4). These are environments where nutrients are more limiting than 
light due to a low supply of nutrients relative to light. In these environments, the plants coexist or show alternative states depending on the consumption traits, where the chance of having alternative states increases when $k_{F} / c_{F}$ relative to $k_{S} / c_{S}$ increases. In light-limited environments on the other hand, the competition outcome is hardly affected: the plants outcompete each other or coexist, and do not show alternative states, irrespective of the consumption traits. Note that the competition outcome does not depend on the absolute values of $k$ and $c$, as long as their ratio $k / c$ is constant, because then the consumption vector does not change (see Eq. A.6 and A.7 in Appendix A). However, the absolute value of $k$ does affect the equilibrium biomass: a higher value for $k$ leads to a lower biomass due to more self-shading.

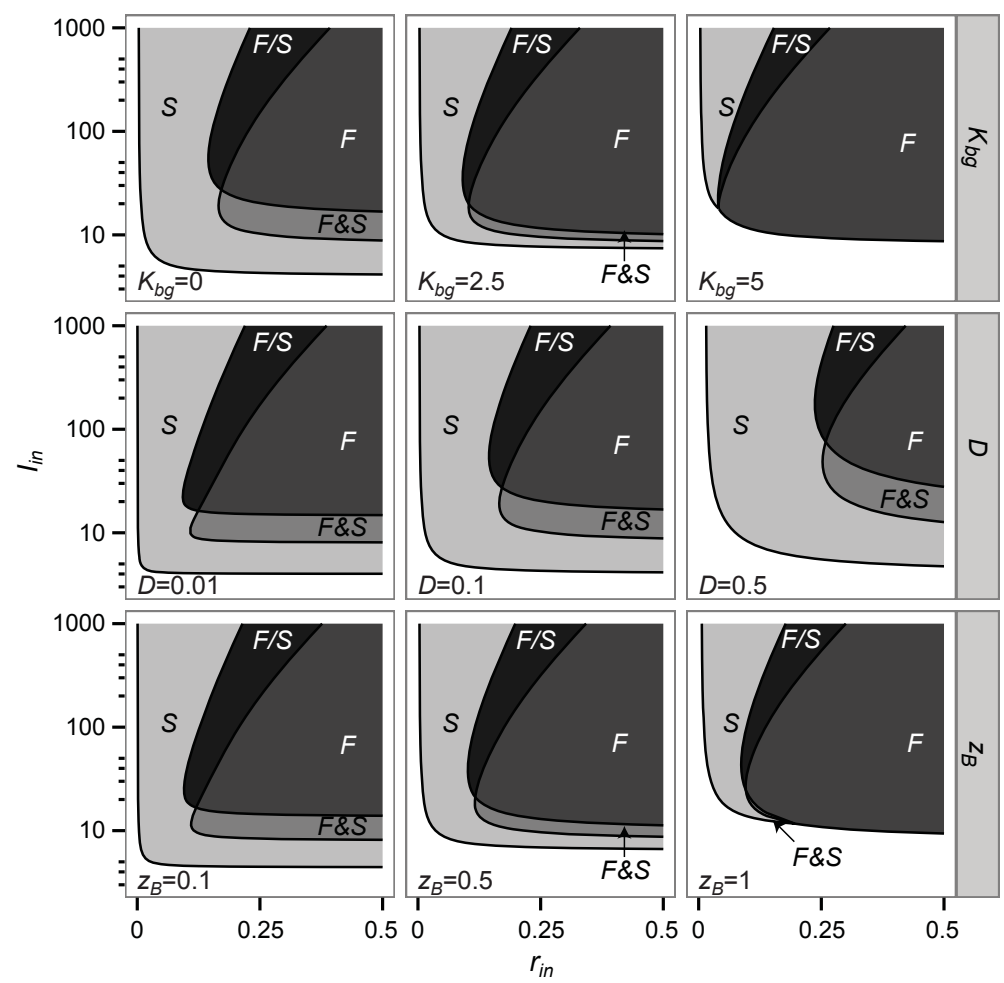

Figure 2.5. Outcome of the competition of floating plant $F$ and submerged plant $S$ for different background light attenuation $K_{b g}$ (upper panels), dilution rate $D$ (middle panels) and water column depth $z_{B}$ (lower panels). The parameter values are the same as in Fig. 2.2D, except for the lower panels where the background attenuation is non-zero $\left(K_{b g}=2\right)$, such that the water column depth influences the average light availability for the submerged plant. 
The importance of background attenuation, dilution rate and water depth Varying the depth, dilution rate and background attenuation of the water illustrates how the environment controls the extent to which species can benefit from their specific position and traits. For example, a higher background light attenuation of the water reduces the competitive success of the submerged plant (Fig. 2.5, upper panels), as the resulting lower light availability in the water column affects the submerged plant only. High background attenuation can even lead to the disappearance of submerged plants in light-limited environments, as the light availability in the water column becomes too low for the plants to persist.

An increased dilution rate enhances the competitive success of the plant with the lowest nutrient requirements (Fig. 2.5, middle panels), which in our case is the submerged plant. This is because a higher dilution rate implies more water flow (see Eq. 2.9) and thus a lower nutrient concentration in the inflow for a given nutrient loading $r_{i n}$. This lower concentration also implies that both plants need more light to persist. An increase of the water depth simultaneously lowers the average light availability in the water column, which is detrimental to the submerged plant, and lowers the nutrient availability as the nutrient loading has to be divided over a larger depth, which in our case is most detrimental to the floating plant. With the current model settings the floating plant suffers less from deeper water (Fig. 2.5, lower panels), as the adverse effect on the submerged plant takes precedence over the adverse effect on floating plants.

\section{Competition outcome for common macrophytes}

When parameterizing the model for floating and submerged plant species common in temperate regions (duckweed: Lemna sp. and waterweed: Elodea sp.) for $\mathrm{N}$ or $\mathrm{P}$ as a limiting nutrient, the model predicts that waterweed outcompetes duckweed at low resource supply, duckweed and waterweed coexist at intermediate resource supply, and duckweed outcompetes waterweed at high resource supply (Fig. 2.6, left panels). Thus, for these species their asymmetrical competition for light does not lead to alternative stable states. We found this pattern to be robust as it is nearly insensitive (not shown here) to the chosen background attenuation and the chosen nutrient content of the plants, where in Fig. 2.6 we used the average of their reported minimal and maximal content. The found pattern is in line with an extensive dataset on observed vegetation in Dutch ditches from 1978 till 2006, where in almost half of the ditches where duckweed or waterweed were observed, they were 
found to coexist (Fig. 2.6, right panel). This combination of model results and field data suggests that duckweed-dominance is unlikely to be an alternative stable state.
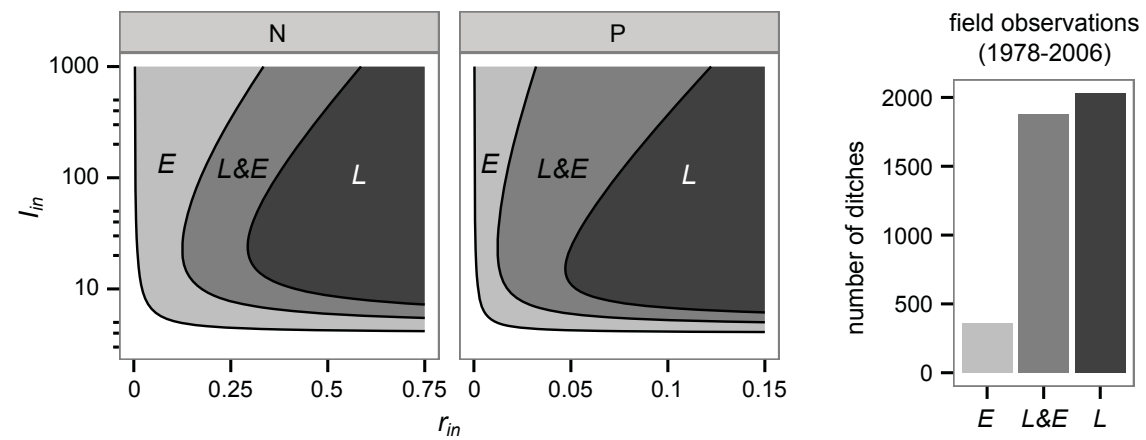

Figure 2.6. Outcome of the competition (left panels) of duckweed L (Lemna sp.) and waterweed $E$ (Elodea sp.) for nitrogen (left: nutrient loading $r_{i n}$ is in $\mathrm{gN} \mathrm{m}^{-2}$ day $^{-1}$ ) and phosphorus (right: $r_{i n}$ is in $\mathrm{gP} \mathrm{m}^{-2}$ day $^{-1}$ ) as a limiting nutrient. The right panel shows the number of ditches in the Netherlands where duckweed and waterweed were observed in absence of each other or in coexistence. For plant parameter values in left panels see table 1. Environmental parameters in left panels: $K_{\text {bg }}=0.5, z_{B}=0.5, D=0.1$.

\subsection{Discussion}

In many places around the world, floating plant dominance is considered to be a true nuisance. Understanding when floating plants dominate, and particularly knowing if their dominance can be a (self-enhancing) alternative stable state, is therefore a relevant issue for ecosystem management. Our model shows that dominance of floating plants cannot be an alternative stable state in light-limited environments: when light-limitation is reduced by an increase of light, the submerged plant - which can cope with the lowest light levels - starts to coexists with and is eventually 'outshaded' by the floating plant. The fact that the plants coexist and do not show alternative stable states can be explained by the requirement for stable coexistence from classical competition theory, namely that intraspecific competition must be greater than interspecific competition (Williamson 1957). In other words, a species can coexist with another species when it suffers more from itself than from the other. In our model, the floating plant does not compete for light 
with the submerged plant and therefore, in light-limited environments, the floating plant always suffers more from itself by self-shading (intraspecific competition) than from the other (interspecific competition), which leads to stable coexistence.

In nutrient-limited environments, the model predicts that with increasing nutrient levels, submerged plants are outcompeted by floating plants. This pattern was also found in field studies and mesocosm studies (Forchhammer 1999; Janes et al. 1996; Netten et al. 2010; Portielje and Roijackers 1995). At intermediate nutrient levels, floating and submerged plants can either coexist or show alternative stable states, depending on their resource consumption. The chance of alternative stable states increases when the submerged plant consumes more nutrients compared to the floating plant (the smaller $k_{S} / c_{S}$ compared to $k_{F} / c_{F}$ ), thus the more the floating plant suffers from the submerged plant than from itself. The semi-mechanistic model of Scheffer et al. (2003) gives comparable results: alternative stable states only occur as long as the submerged plant consumes more nutrients (per unit biomass) than the floating plant. Their model predicts no alternative stable states when the plants consume equal amounts of nutrients, which is not per se the case in our model: we found that for equal consumption traits $\left(k_{S} / c_{S}=k_{F} / c_{F}\right)$ the plants can still show alternative stable states (Fig. 2.2) or coexist (not shown here), depending on plant traits other than resource consumption.

Interestingly, for increasing supply of nutrients and light, the model predicts that the floating plant due to its primacy for light can coexist with - or eventually outcompete - the submerged plant, even when the submerged plant has the lowest resource requirements. This is not possible in unlayered communities, as described in $I_{\text {out }}^{*}-R^{*}$ theory (Huisman and Weissing 1995), where the species with the lowest minimal resource requirements always outcompetes the other ( $R^{*}$ rule). Another difference is that consumption traits that lead to coexistence in unlayered communities, namely such that each species consumes more of the resource that most limits its growth, may in our layered community lead to alternative stable states, and vice versa (see Fig. 2.4 where $k_{F} / c_{F}<k_{S} / c_{S}$ may lead to alternative stable states and $k_{F} / c_{F}>k_{S} / c_{S}$ may lead to coexistence). Related to this, the layeredness of species leads to a richer competition outcome in a sense that the same two species can, depending on the environmental conditions, coexist or show alternative stable states, whereas in unlayered communities the same two species either coexist or show alternative stable states. 
Thus, compared to unlayered communities as described in $I_{\text {out }}^{*}-R^{*}$ theory, the layeredness - and associated asymmetry in competition for light leads to fundamentally different results. Appendix B illustrates this by showing why the graphical isocline approach, which is used in $I_{\text {out }}^{*}-R^{*}$ theory and also in $R^{*}$ theory (Tilman 1980) to predict the competition outcome, does not apply to layered communities.

Interestingly, more complex phytoplankton models of asymmetrical competition predict similar differences, although in these cases there are, next to the primacy for light, more mechanisms at play which could cause these differences. For example, in the model of Jäger and Diehl (2014) asymmetrical competition for nutrients co-determines the competition outcome. In their double-asymmetric model not only the upper species (pelagic algae) have the primacy for light, but also the lower species (benthic algae) have the primacy for nutrients, as they assumed that the nutrients are supplied from below. Furthermore, Ryabov and Blasius (2011) found that differences in the dispersal ability of pelagic algae make that a species with lower resource requirements can be outcompeted by a species with higher dispersal ability. In addition, the non-uniform nutrient environment in these models of pelagic algae - due to limited mixing of the water combined with nutrient supply from below - was found to explain that consumption traits that lead to coexistence in unlayered communities may lead to alternative stable states and vice versa (Ryabov and Blasius 2011; Yoshiyama et al. 2009). However, our study shows that even in a uniform nutrient environment, i.e. no nutrient gradient with depth, similar results can be found. We show that the most parsimonious form of asymmetrical competition, being asymmetrical competition for light while maintaining symmetry in competition for nutrients in a homogeneous nutrient environment, is sufficient to cause fundamentally different results compared to unlayered communities.

Our theoretical framework can also be used to study the competition between free-floating plants and phytoplankton. De Tezanos Pinto and O'Farrell (2014) state that field studies suggest that floating plants and phytoplankton show alternative stable states, however that mathematical models are needed for more conclusive evidence. Our model may also be useful to study resource competition of terrestrial plants having different canopy positions.

For floating and submerged plant species that are common in temperate regions (duckweed: Lemna sp. and waterweed: Elodea sp.), both model and field data suggest that the occurrence of alternative stable states is unlikely. Instead, the species coexist or outcompete each other. This implies 
that floating-plant dominance may be a less persistent ecosystem state than suggested by Scheffer et al. (2003). We found similar model results (not shown here) when parameterizing both species directly from literature, instead of from the ecosystem model PCDitch (Janse 1998). For both parameterizations we kept the light attenuation coefficient $k$ of Lemna sp. equal to that of Elodea sp. because little is known about $k$. In the model, we assumed that submerged plants only take up nutrients from the water column, whereas most submerged plants, like waterweed, can also take up nutrients from the sediment by their roots (Barko et al. 1991). Taking this into account will probably reduce the chance of alternative stable states even further, as a floating plant does not suffer from a submerged plant that takes up nutrients from the sediment only, which promotes coexistence instead of alternative stable states. We probably overestimated the nutrient loading needed for dominance of Lemna sp. because our model does not take into account nutrient recycling by water plants due to dying-off. These processes, as well as other important phenomena in aquatic ecosystems such as variable stoichiometry, seasonal dynamics in light, nutrients, temperature and phenology are accounted for in more complex models such as PCDitch (Janse 1998), however, at the cost of general insights in the dominant mechanisms and the way they interact.

\subsection{Conclusions}

The model presented here provides general insights in the competition for light and nutrients in layered communities of aquatic plants. At high supply of light and nutrients, floating plants always outcompete submerged plants due to their primacy for light, even when submerged plants have lower resource requirements. The occurrence of coexistence or alternative stable states depends on the environmental conditions: in light-limited environments the plants do never show alternative stable states, whereas in nutrient-limited environments the plants coexist or show alternative stable states depending on their resource consumption traits. Compared to unlayered communities, the layeredness - and associated asymmetry in competition for light - leads to fundamentally different results: the competition outcome can no longer be predicted from species traits such as minimal resource requirement $\left(R^{*}\right.$ rule) and resource consumption. Also it leads to a richer competition outcome in a sense that the same two species can, depending on the environment, coexist or show alternative stable states. For two common plant species in temperate regions both the model and field data suggest that floating-plant dominance 
is unlikely to be a (self-enhancing) alternative stable state. This would ease ecosystem management that aims at reducing floating-plant dominance.

\section{Acknowledgements}

We thank Annette Janssen, Jan Janse and four anonymous reviewers for their helpful comments during the development of this paper. This work is financed by the Netherlands Foundation for Applied Water Research (STOWA) project no. 443237. This is publication 5798 of the Netherlands Institute of Ecology (NIOO-KNAW). 


\section{Appendix A: Species' minimal resource requirements and resource consumption vector}

The plant's lowest required nutrient concentration $R_{\min }$ follows from the requirement for persistence $p_{i}=m_{i}$ (see Eq. 2.1 and 2.2) when light is nonlimiting $\left(f_{I}=1\right)$. Using Eq. 2.3 and 2.4 this leads to

$$
R_{\min , i}=\frac{m_{i} M_{i}}{p_{\max , i}-m_{i}} .
$$

The derivation of the minimal required light intensity $I_{\min }$ is less straight forward, as $I_{\min }$ is reached only when there is no self-shading, thus when the equilibrium biomass approaches zero. Furthermore, for submerged plants $I_{\min }$ also depends on the background light attenuation of the water. $I_{\min }$ follows from $p_{i}=m_{i}$ when nutrients are non-limiting $\left(f_{R}=1\right)$, and in case the background attenuation is zero it follows from Eq. 2.3, 2.5 and 2.6 that

$$
I_{\min , i}=\frac{m_{i} H_{i}}{p_{\max , i}-m_{i}} .
$$

as for this case Eq. 2.5 and 2.6 read

$$
\lim _{\text {biomass } \rightarrow 0} f_{I, i}=\frac{I}{I+H_{i}} .
$$

The consumption vector can be derived by expressing the equilibrium biomass in terms of nutrients and light. For floating plants at monoculture equilibrium $F^{*}$ this means that $\mathrm{d} F / \mathrm{d} t=0$ (Eq. 2.1) and $\mathrm{d} R / \mathrm{d} t=0$ (Eq. 2.9) leading to a biomass expression in terms of nutrients:

$$
F^{*}=\frac{r_{i n}-z_{B} D R^{*}}{c_{F} m_{F}}
$$

where the stars denote the equilibrium state. The equilibrium biomass $F^{*}$ can also be expressed in terms of light (following from Eq. 2.7):

$$
F^{*}=\frac{\ln \left(I_{i n}\right)-\ln \left(I_{0}^{*}\right)}{k_{F}} .
$$

The consumption vector results from combining both expressions for $F^{*}$ (Eq. A.4 and A.5) and substituting expressions for $r_{i n}$ and $I_{i n}$ based on the resource supply point, which represents the highest possible resource levels 
in equilibrium (Tilman 1980) that are achieved in a system without plants ( $I_{i n}=I_{0}$ and $r_{i n}=z_{B} D R$ following from Eq. 2.7 and 2.9):

$$
\ln \left(I_{0}\right)=\frac{k_{F}}{c_{F} m_{F}} z_{B} D\left(R-R^{*}\right)+\ln \left(I_{0}^{*}\right) .
$$

Thus the consumption vector represents how the nutrient concentration $R$ and the light intensity at the lower end of the plant $I_{0}$ are reduced to their equilibrium levels due to plant consumption. Similarly, the consumption vector of the submerged plant can be derived which leads to

$$
\ln \left(I_{\text {out }}\right)=\frac{k_{S}}{c_{S} m_{S}} z_{B} D\left(R-R^{*}\right)+\ln \left(I_{\text {out }}^{*}\right) .
$$

The slope of the consumption vector in the $\ln \left(I_{0}\right)-R$ and $\ln \left(I_{\text {out }}\right)-R$ plane, respectively, which for both plants equals $\left(k_{i} / c_{i} m_{i}\right) z_{B} D$, indicates the factors that control plant resource consumption. As a result, the resource consumption is controlled by both environmental conditions (water column depth $z_{B}$ and dilution rate $D$ ) and species traits (light attenuation coefficient $k$, nutrient content per unit biomass $c$ and loss rate $m$ ).

\section{Appendix B: Unpredictability of competition outcome in layered communities}

An elegant feature of consumer-resource competition in uniform environments, described by $R^{*}$ theory for nutrients (Tilman 1980; Tilman 1982) and $I_{\text {out }}^{*}-R$ theory for light and nutrients (Huisman and Weissing 1995), is that one can predict the competition outcome based on species traits. This is done by a graphical isocline approach, based on the species their consumption vectors and zero net growth isoclines (ZNGI's), and the resource supply point. The ZNGI gives combinations of resource levels, i.e. nutrient concentration $R$ and light intensity at the bottom of the water column $I_{\text {out }}$, at which the net growth of the species is zero, and the resource supply point represents the highest possible resource levels in equilibrium that are reached in an empty system.

The species with the lowest resource requirements, and thus the lowest ZNGI, is the best competitor ( $R^{*}$ rule) as it depletes the limiting resources to the lowest level and therefore outcompetes all other species. When one species is a better competitor for resource 1 and the other species is a better competitor for resource 2 (i.e. their ZNGI's intersect), the species coexist or 
show alternative stable states when the resource supply point lies within the region bounded by their consumption vectors (Fig. B.1, upper panels). They coexist when each species consumes more of the resource that most limits its growth. Otherwise they show alternative stable states.

In layered communities of species competing for light and nutrients, the competition outcome can no longer be predicted based on species traits. This can be graphically illustrated by regarding the ZNGI's that now change in presence of the other species. This is because the ZNGI depends on the light supply and the light supply for the lower species is lowered in presence of the upper species. Therefore, the ZNGI of the lower species moves upward due to shading, as a lower light supply shifts the ZNGI away from the origin in the $R-I_{\text {out }}$ plane (Huisman and Weissing 1994). This is because a lower light supply leads to a higher light intensity at the bottom $\left(I_{\text {out }}\right)$ since the decrease in light absorption due to lower biomass overcompensates for the effect of the decreased light supply. The ZNGI of the upper species is not affected in presence of the lower species, at least when expressed in terms of the outgoing light intensity at its lower end ( $I_{0}$ for floating plants, see Fig. 1 ). However, the ZNGI moves downward when expressing it in terms of the light intensity at the bottom of the water column $I_{\text {out }}$ since $I_{\text {out }}$ is lower than $I_{0}$ in presence of the lower species.

The change of both ZNGI's (see arrows in Fig. B.1) is beneficial to the upper species illustrating its competitive advantage: due to its primacy for light it may coexist with (Fig. B.1A and B), show alternative stable states with (Fig. B.1C) - or even outcompete - the submerged plant, even when the submerged plant has the lowest resource requirements in monoculture.

Furthermore, in layered communities the occurrence of alternative stable states or coexistence, can no longer be predicted based on the resource consumption traits. Consumption vectors that lead to stable coexistence for unlayered communities may lead to alternative stable states in unlayered communites (Fig. B.1C), or vice versa (Fig. B.1B). Note that the species stably coexist when the coexistence equilibrium $\left(R^{*}, I_{\text {out }}^{*}\right)$, given by the intersection of both ZNGI's, is stable, and that the species show alternative stable states when this equilibrium is unstable. Even for the exceptional case that both species have equal consumption traits, the species may stably coexist (Fig. B.1A) or show alternative stable states in layered communities, contrary to unlayered communities where species can only outcompete each other when having equal consumption traits. Then, the stable coexistence in layered communities results from the fact that the species adjust their equilibrium 
biomass such that the intersection of the ZNGI's is linked to the resource supply point by the consumption vectors.
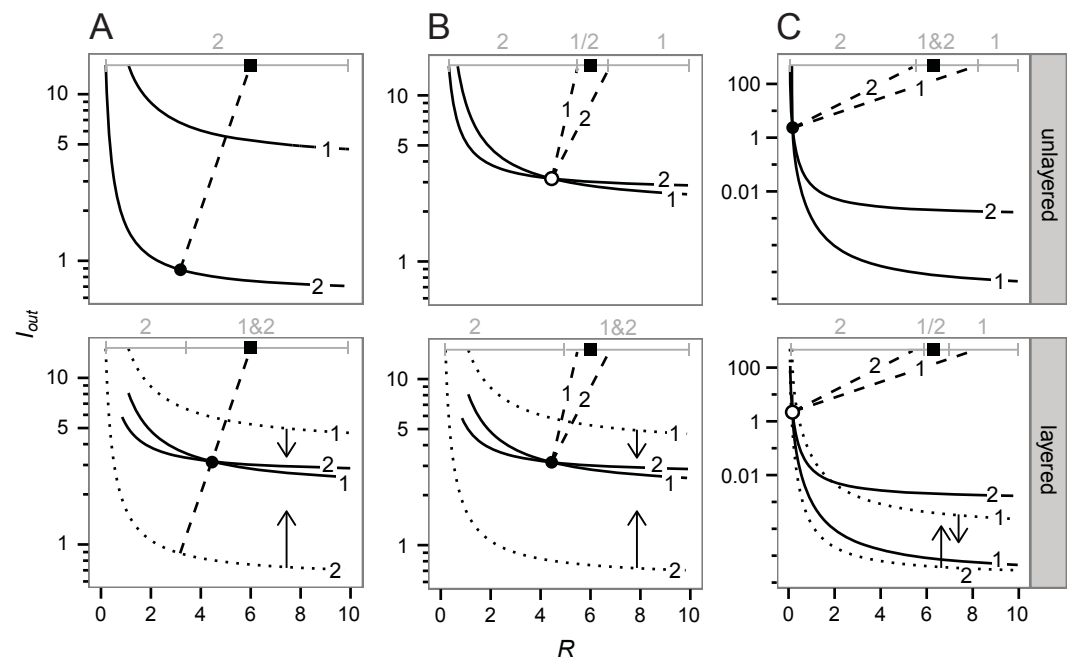

Figure B.1. Graphical isocline approach for two species competing for light and nutrients in an unlayered community sensu $I_{\text {out }}^{*}-R^{*}$ theory (upper panels) and in a layered community (lower panels; $1=$ floating plant, $2=$ submerged plant) for three different settings (panels A to C), plotted in the $R-I_{\text {out }}$ plane where $R$ is the nutrient concentration in the water and $I_{\text {out }}$ is the light intensity at the bottom of the water column. It comprises the ZNGI's (dotted and solid lines), consumption vectors (dashed lines), resource supply point (square), coexistence equilibrium (solid dot $=$ stable equilibrium, open dot $=$ unstable equilibrium which implies alternative stable states) and competition outcome (grey lines) for resource supply points with different nutrient supply (changing the light supply would lead to other results as the ZNGI's depend on the light supply). Note that in the layered community, contrary to unlayered communities, the ZNGI of a species changes in presence of the other species (indicated by the arrows): the dotted line gives the ZNGI in monoculture and the solid line gives the ZNGI at equilibrium density of the other species as realized at the resource supply indicated by the square. Also note that in panels B and C the species traits differ between the unlayered and the layered community. Therefore in these panels, the ZNGI's in the unlayered community differ from the ZNGI's in monoculture in the layered community. However, the resource consumption traits are the same in both communities, as indicated by the similar consumption vectors, where in panel A the consumption vectors of both species coincide. Parameter settings for the layered community are the same as in Fig. 2.4 (panel A and B: $I_{i n}=15, r_{i n}=0.3$ and for panel A $k_{F} / c_{F}=k_{S} / c_{S}=1$ and for panel B $k_{F} / c_{F}=0.67$ and $k_{S} / c_{S}=1$; panel C: $k_{F} / c_{F}=1.5, k_{S} / c_{S}=0.67, I_{i n}=500$ and $\left.r_{i n}=0.315\right)$. 



\title{
Chapter 3
}

\section{$\mathrm{N}_{2}$-fixation in aquatic ecosystems does not axiomatically lead to $P$ limitation}

\author{
van Gerven, L. P. A., J.H. Janse, J. J. Kuiper, W. M. Mooij, H. W. Paerl and J. J. M.
} de Klein. $\mathrm{N}_{2}$-fixation in aquatic ecosystems does not axiomatically lead to $\mathrm{P}$ limitation. Submitted.

Abstract. A long standing debate in ecology deals with the role of nitrogen and phosphorus in management and restoration of aquatic ecosystems. It has been argued that nutrient reduction strategies should solely focus on $\mathrm{P}$, as reducing $\mathrm{N}$ inputs is ineffective because $\mathrm{N}_{2}$-fixing species will eventually correct for $\mathrm{N}$ deficits, perpetuating $\mathrm{P}$ limitation. A sound mechanistic understanding of this principle is, however, lacking. Here we use mechanistic resource competition theory, an ecosystem model and a 45-year field data set on eutrophic floating-plant dominated ecosystems to show that $\mathrm{N}_{2}$-fixation is unlikely to perpetuate $\mathrm{P}$ limitation. This is explained by $\mathrm{N}_{2}$-fixers typically requiring higher $\mathrm{P}$ concentrations to persist than non- $\mathrm{N}_{2}$-fixers, implying that they cannot deplete the $\mathrm{P}$ concentration enough for the non- $\mathrm{N}_{2}$-fixing eutrophic community to become P-limited. These findings provide a mechanistic basis for the need to consider the reduction of both $\mathrm{N}$ and $\mathrm{P}$ inputs to most effectively restore nutrient over-enriched aquatic ecosystems.

\subsection{Introduction}

Eutrophication due to anthropogenic nutrient over-enrichment threatens the biodiversity and functioning of freshwater ecosystems worldwide (Smith et al. 1999), by promoting toxic algal blooms (Anderson et al. 2002) and the invasion by dense mats of free-floating plants (Scheffer et al. 2003). Both lead to dark, anoxic sub-surface conditions that severely constrain aquatic 
life (Heisler et al. 2008). To combat eutrophication, the question arises as to whether to focus on reducing inputs of $\mathrm{N}$ or $\mathrm{P}$ or both (Conley et al. 2009).

Based on evidence from long-term whole-ecosystem experiments, it has been postulated that nutrient reduction strategies should focus solely on $\mathrm{P}$, because $\mathrm{N}$ reduction favours $\mathrm{N}_{2}$-fixing cyanobacteria that draw on the vast atmospheric $\mathrm{N}_{2}$ reserve, eventually providing the eutrophic ecosystem with enough $\mathrm{N}$ to compensate $\mathrm{N}$ deficits, thus perpetuating P-limitation (Hecky and Kilham 1988; Schindler 1977; Schindler et al. 2008; Schindler and Hecky 2009). The general applicability of this principle has been questioned however, as it has been argued that $\mathrm{N}_{2}$-fixers cannot meet the $\mathrm{N}$ requirements of eutrophic ecosystems due to ecological and geochemical constraints on $\mathrm{N}_{2}$-fixation (Howarth et al. 1988; Paerl 1990), and because the $\mathrm{N}$ input by $\mathrm{N}_{2}$-fixers is offset by denitrification (Lewis and Wurtsbaugh 2008; Lewis et al. 2011; Paerl and Scott 2010; Scott and McCarthy 2010), both leading to Nlimitation. A far-reaching implication is that ecosystem managers should also consider the reduction of $\mathrm{N}$ inputs, rather than focusing solely on $\mathrm{P}$ (Conley et al. 2009). Interestingly, thus far, a thorough mechanistic understanding of how $\mathrm{N}_{2}$-fixation affects nutrient limitation has been missing from this debate.

Here, we used mechanistic resource competition theory (Huisman and Weissing 1995; Tilman 1982) to systematically explore whether $\mathrm{N}_{2}$-fixers have the capacity to perpetuate $\mathrm{P}$ limitation when lowering $\mathrm{N}$ supply. First, we built a resource competition model, to analyse for different combinations of $\mathrm{N}$ and $\mathrm{P}$ supply how $\mathrm{N}_{2}$-fixation influences the structure and nutrient limitation characteristics of a eutrophic community of floating plants. Then we repeated the analysis but now in a more realistic environment, by using an established complex ecosystem model. Next, we compared the model predictions with a 45-year field data set on eutrophic floating-plant dominated ecosystems.

\subsection{Methods}

\section{The resource competition model}

The model describes floating plants $A$ (or $C$ ) and $L$ (or $B$ ) that compete for $\mathrm{N}, \mathrm{P}$ and light. Only plant $A$ is able to fix $\mathrm{N}_{2}$ (Table 3.1, Fig. S1). The model is based on theoretical frameworks for resource competition (Huisman and Weissing 1995; Van Gerven et al. 2015a) and simulates the dynamics of plant biomass $\left(\mathrm{gDW} \mathrm{m}^{-2}\right.$ ) and the $\mathrm{N}$ and $\mathrm{P}$ concentrations $\left(\mathrm{gN} \mathrm{m}^{-3}\right.$ and $\left.\mathrm{gP} \mathrm{m}^{-3}\right)$ in the water (Eqs. 3.1-3.4). The $\mathrm{N}$ and $\mathrm{P}$ concentrations are affected by supply, outflow, uptake and remineralization. 
Table 3.1. The resource competition model.

Biomass and nutrient dynamics

$\frac{\mathrm{d} L}{\mathrm{~d} t}=\left(p_{L}-m_{L}\right) L$

$\frac{\mathrm{d} A}{\mathrm{~d} t}=\left(p_{A}-m_{A}\right) A$

$\frac{\mathrm{d} N}{\mathrm{~d} t}=\frac{n_{i n}}{z_{B}}-D N-\frac{1}{z_{B}}\left(c_{N, L} p_{L} L\right)+\frac{\alpha}{z_{B}}\left(c_{N, L} m_{L} L+c_{N, A} m_{A} A\right)$

$\frac{\mathrm{d} P}{\mathrm{~d} t}=\frac{p_{i n}}{z_{B}}-D P-\frac{1}{z_{B}}\left(c_{P, L} p_{L} L+c_{P, A} p_{A} A\right)+\frac{\alpha}{z_{B}}\left(c_{P, L} m_{L} L+c_{P, A} m_{A} A\right)$

Auxiliary equations

$p_{L}=\min \left[f_{N, L}, f_{P, L}\right] f_{I, L} p_{\max , L}$

$p_{A}=f_{P, A} f_{I, A} p_{\max , A}$

$f_{N, L}=\frac{N}{N+M_{N, L}}$

$f_{P, i}=\frac{P}{P+M_{P, i}}$

$f_{I, i}=\frac{1}{k_{L} L+k_{A} A} \int_{I_{\text {out }}}^{I_{\text {in }}} \frac{1}{H_{i}+I} \mathrm{~d} I=\frac{1}{k_{L} L+k_{A} A} \ln \left(\frac{H_{i}+I_{\text {in }}}{H_{i}+I_{\text {out }}}\right)$

$I_{\text {out }}=I_{\text {in }} e^{-\left(k_{L} L+k_{A} A\right)}$

Species parameters

$p_{\max , i} \quad$ Maximum growth rate $\left(\mathrm{day}^{-1}\right)$

$m_{i} \quad$ Loss rate $\left(\right.$ day $\left.^{-1}\right)$

$k_{i} \quad$ Light attenuation coeff. $\left(\mathrm{m}^{2} \mathrm{gDW}^{-1}\right)$

$H_{i} \quad$ Half-sat. constant for light $\left(\mathrm{J} \mathrm{m}^{-2} \mathrm{~s}^{-1}\right)$

$M_{N, L} \quad$ Half-sat. constant for $\mathrm{N}\left(\mathrm{gN} \mathrm{m}^{-3}\right)$

$M_{P, i} \quad$ Half-sat. constant for $\mathrm{P}\left(\mathrm{gP} \mathrm{m}^{-3}\right)$

$c_{N, i} \quad \mathrm{~N}$ to dry weight ratio $\left(\mathrm{gN} \mathrm{gDW}^{-1}\right)$

$c_{P, i} \quad$ P to dry weight ratio $\left(\mathrm{gP}_{\mathrm{gDW}} \mathrm{gD}^{-1}\right)$

Environmental parameters

$n_{i n} \quad \mathrm{~N}$ loading $\left(\mathrm{gN} \mathrm{m}^{-2}\right.$ day $\left.^{-1}\right)$

$p_{\text {in }} \quad$ Ploading $\left(\mathrm{gP} \mathrm{m}^{-2}\right.$ day $\left.^{-1}\right)$

$I_{i n} \quad$ Incident light intensity $\left(\mathrm{J} \mathrm{m}^{-2} \mathrm{~s}^{-1}\right)$

$z_{B} \quad$ Water column depth (m)

$D \quad$ Dilution rate of water column $\left(\right.$ day $\left.^{-1}\right)$

Remineralization fraction of died-off plants (-)

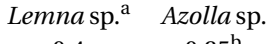

$\begin{array}{cc}0.4 & 0.25^{\mathrm{b}} \\ 0.05 & 0.03^{\mathrm{c}} \\ 0.07 & 0.07^{\mathrm{d}} \\ 25 & 35^{\mathrm{e}} \\ 2.5 & - \\ 0.25 & 0.45^{\mathrm{f}} \\ 0.07 & 0.03^{\mathrm{g}} \\ 0.015 & 0.01^{\mathrm{g}}\end{array}$

${ }^{a}$ Janse $1998,{ }^{b}$ van der Heide et al. (2006), ${ }^{c}$ chosen such that maximum biomass is realistic, ${ }^{d}$ Forchhammer (1999), ${ }^{\mathrm{e}}$ Moretti and Siniscalco Gigliano (1998), ${ }^{\mathrm{f}}$ Bieleski and Lauchli (1992), ${ }^{\mathrm{g}}$ Costa et al. (1999).

The biomass dynamics depend on loss rate $m$ and growth rate $p$. The growth is co-limited by light and nutrients (Eqs. 3.5-3.6). Nutrient limitation follows the Michaelis-Menten function (Eqs. 3.7-3.8). Non- $\mathrm{N}_{2}$-fixer $L$ is limited by either $\mathrm{N}$ or $\mathrm{P}$ according to Liebig's law of the minimum, whereas $\mathrm{N}_{2}$-fixer $A$ is only limited by $\mathrm{P}$ as its $\mathrm{N}$ demand is fulfilled by $\mathrm{N}_{2}$-fixation. 
Light limitation is also characterized by the Michaelis-Menten function (Eq. 3.9) and follows from integrating over the plant depth, as the light intensity $I$ decreases with depth due to light attenuation by the plants according to Lambert Beer's law (Eq. 3.10).

\section{Complex ecosystem model}

The used complex ecosystem used consists of the used resource competition model embedded in PCDitch (Janse 1998), a full-scale and well-tested dynamic ecosystem model that has been successfully applied to predict the nutrient-driven regime shift from submerged plants to floating-plant dominance (Van Gerven et al. 2015b and references therein). PCDitch includes several functional groups of water plants that compete for nutrients and light and have a different competition strategy due to their distinct growth forms (Fig. S2). Furthermore, the model comprises a sediment layer, includes all key nutrient processes like sediment-P release and denitrification, and accounts for seasonal variation in water temperature, light intensity and day length (see Janse 2005 for detailed process descriptions). For PCDitch, we used the parameter settings of the calibrated model (Janse 1998), resembling an 'average' macrophyte-dominated freshwater ecosystem in the temperate zone. We slightly extended the resource competition model part of the ecosystem model to account for the phenology of Lemna and Azolla, by making the growth temperature-dependent and incorporating an overwintering period and a growing season. We adopted the associated phenological parameters from the original Lemna group in PCDitch, and kept these parameters the same for Lemna and Azolla.

\subsection{Results}

When considering hypothetical $\mathrm{N}_{2}$-fixers and non- $\mathrm{N}_{2}$-fixers that have the same resource requirements, the resource competition model predicts that $\mathrm{N}_{2}$-fixers indeed have the potential to keep the system P-limited when the system's $\mathrm{N}$ supply is lowered. This can be seen in Fig. 3.2, showing that Plimitation is more pronounced in presence of a $\mathrm{N}_{2}$-fixer (Fig. 3.1B) compared to a community of only non- $\mathrm{N}_{2}$-fixers (Fig. 3.1A). This is because the $\mathrm{N}_{2}$ fixer can alleviate $\mathrm{N}$-limitation by not taking up $\mathrm{N}$ from the water and by 'fertilizing' the water with fixed atmospheric $\mathrm{N}$ through remineralization of died-off biomass. Hence, the non- $\mathrm{N}_{2}$-fixer remains P-limited with decreasing $\mathrm{N}$ supply. This is even the case when there is no 'N-fertilization' (Fig. S3). 


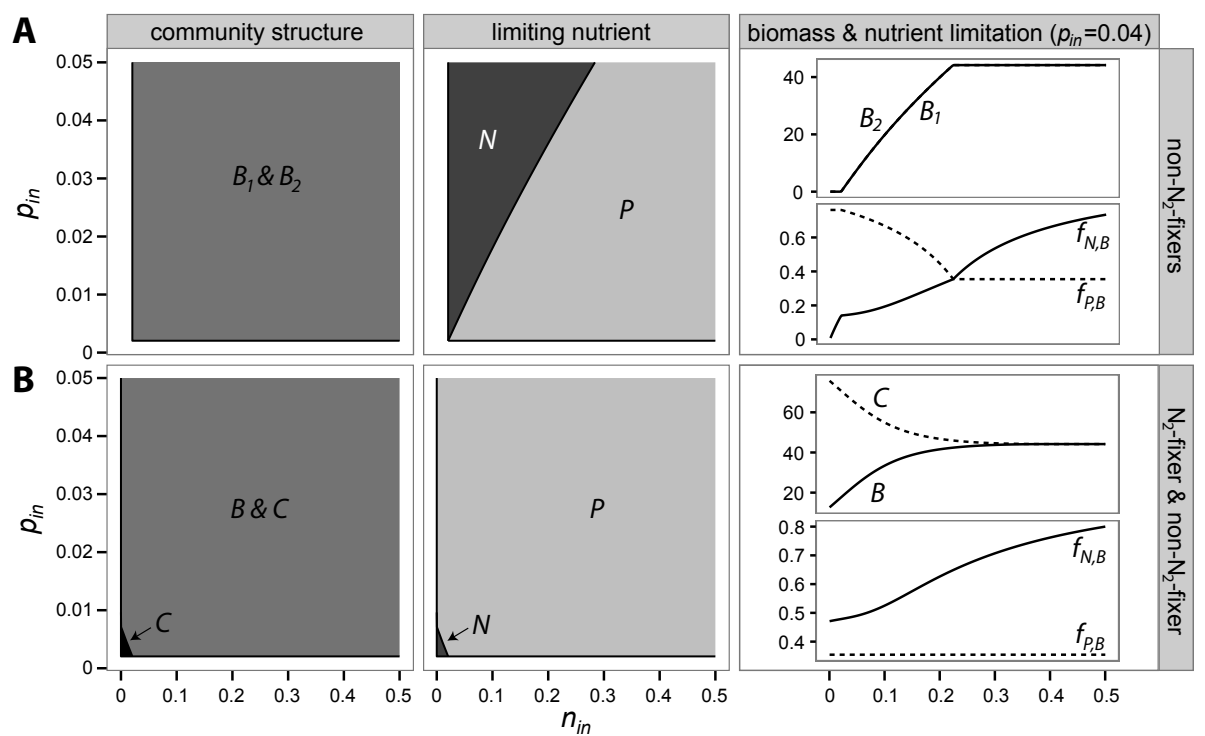

Figure 3.1. Predicted structure and nutrient limitation of a community of identical (A) non- $\mathrm{N}_{2}$-fixers $B_{1}$ and $B_{2}$ and (B) non- $\mathrm{N}_{2}$-fixer $B$ and $\mathrm{N}_{2}$-fixer $C$, for different supply of $\mathrm{N}\left(n_{i n}\right)$ and $\mathrm{P}\left(P_{i n}\right)$. Left panel, competition outcome where '\&' denotes coexistence. Middle panel, nutrient that limits the growth of the non- $\mathrm{N}_{2}$-fixer (Eq. 3.5). Right panel, underlying biomasses in gDW $\mathrm{m}^{-2}$ and nutrient limitation factors of the non- $\mathrm{N}_{2}$-fixer (Eqs. 3.7-3.8) along a gradient of $\mathrm{N}$ supply $\left(p_{i n}=0.04\right)$. All species were parametrized as Lemna sp. (Table 3.1) except for $M_{N}$ which does not apply to $\mathrm{N}_{2}$-fixer $C$.

A different picture arises however when we parameterize the resource competition model for 'real' species; the floating plant Lemna (duckweed), which is not able to fix $\mathrm{N}_{2}$, and the water fern Azolla, living in symbiosis with the $\mathrm{N}_{2}$-fixing cyanobacterium Anabaena azollae (Wagner 1997). We then find that $\mathrm{N}_{2}$-fixation can no longer keep the system P-limited at low $\mathrm{N}$ supply (Fig. 3.2A). This is because Azolla has higher resource requirements than Lemna, and therefore cannot deplete the $\mathrm{P}$ concentration to a level low enough for Lemna to become P-limited. This is reflected by the $P^{*}$ and $I_{\text {out }}{ }^{*}$, important traits in competition theory indicating the lowest level to which a species can deplete the $\mathrm{P}$ concentration and light availability in the water (Huisman and Weissing 1995; Tilman 1982). Given Azolla's higher values for $P^{*}$ and $I_{\text {out }}{ }^{*}$, Azolla is an inferior competitor for $\mathrm{P}$ and light. As a result, Lemna competitively excludes Azolla when P is limiting, implying that Lemna cannot be P-limited in presence of Azolla (see Fig. S4 for a graphical explanation). The 


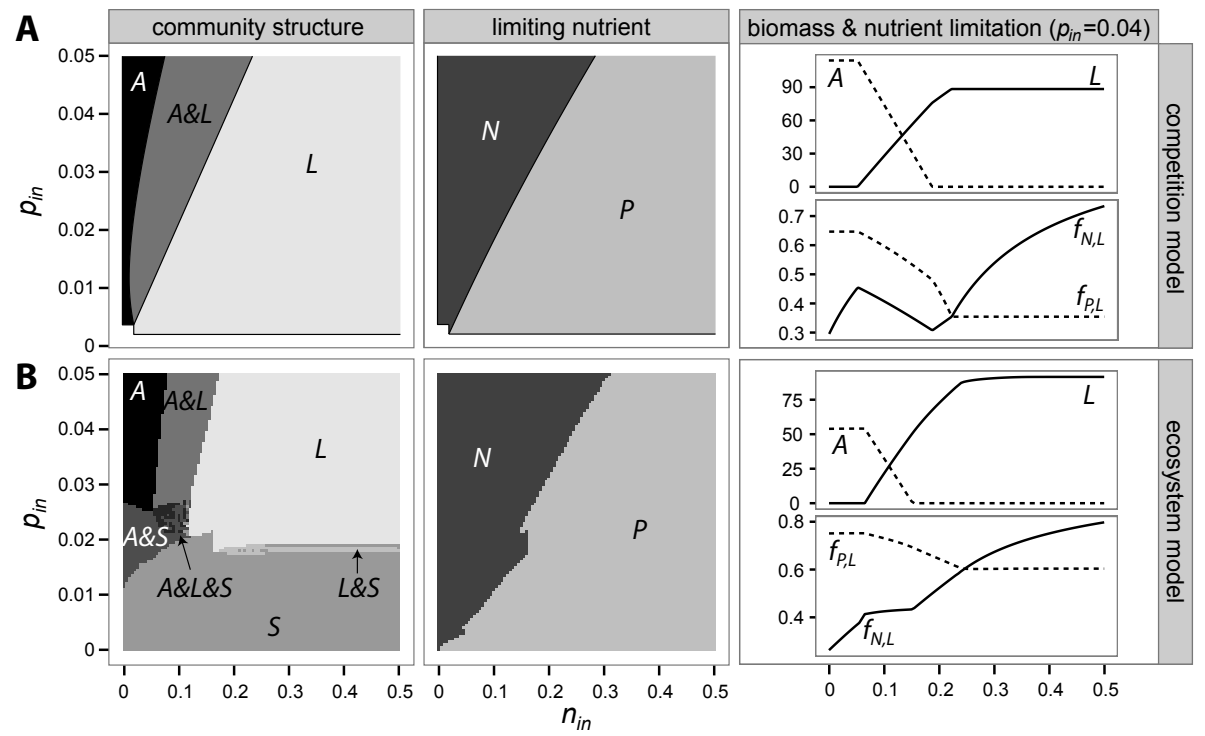

Figure 3.2. Structure and nutrient limitation of a floating-plant community of Lemna $(L)$ and Azolla (A) as predicted by (A) the resource competition model and (B) the complex ecosystem model. Left panel, competition outcome where ' $S$ ' denotes submerged plants. Middle panel, nutrient that limits the growth of the non- $\mathrm{N}_{2}$ fixing Lemna. Right panel, underlying biomasses in $\mathrm{gDW} \mathrm{m}^{-2}$ and nutrient limitation factors of Lemna along a gradient of N supply ( $p_{i n}=0.04$ ). See Table 3.1 for parameter values. Note that for the ecosystem model it concerns summer-averaged results.

limited competitive power of Azolla, being only a superior competitor for $\mathrm{N}$ due to its ability to fix $\mathrm{N}_{2}$, also explains why Azolla is abundant only when the supply of $\mathrm{N}$ is sufficiently low compared to the $\mathrm{P}$ supply (Fig. 3.2A, left panel). These findings remain even if we assume the remineralization and associated 'N-fertilization' to be very high (Fig. S5).

To test if these results hold true in a more realistic environment, we embedded the used resource competition model in the established ecosystem model PCDitch (Janse 1998) that includes additional biota (e.g. algae and submerged plants), seasonality and important nutrient cycling processes like denitrification and sediment P-release. We found the same qualitative results: the $\mathrm{N}_{2}$-fixing Azolla cannot keep the ecosystem P-limited when lowering the $\mathrm{N}$ supply (Fig. 3.2B), even when its 'N-fertilization' is very high (Fig. S6).

Thus, both models predict that $\mathrm{N}_{2}$-fixation does not lead to P-limitation, due to the underlying mechanism that $\mathrm{N}_{2}$-fixers require higher $\mathrm{P}$ concentra- 
tions to persist. These findings are supported by an extensive 45 -year data set on eutrophic floating-plant dominated ecosystems, as analysis of the field data showed that $\mathrm{N}_{2}$-fixing Azolla spp. indeed occur at significantly higher $\mathrm{P}$ concentrations than Lemna spp. (Fig. 3.3A: $p<0.001$, Welch's t-test).

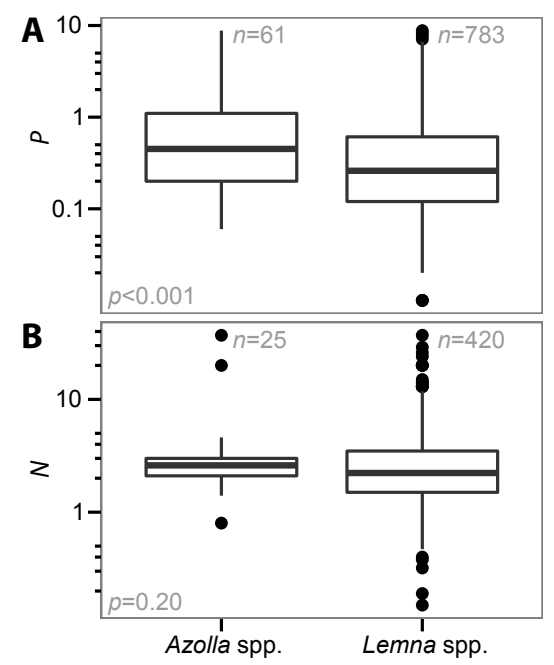

Figure 3.3. Box-whisker plots of (A) the total $\mathrm{P}$ concentrations and (B) the total $\mathrm{N}$ concentrations at which Azolla spp. and Lemna spp. were observed in Dutch drainage ditches (1968 - 2012). The shown $p$-values result from a Welch's t-test for which the data were log-transformed.

\subsection{Discussion}

Although illustrated here for floating plants, it seems plausible that $\mathrm{N}_{2}$-fixers in general have higher resource requirements than their non- $\mathrm{N}_{2}$-fixing competitors, given the energetic costs of $\mathrm{N}_{2}$-fixation and the associated heterocyst production (Paerl 1990). This would imply that also in phytoplanktondominated systems, such as eutrophic lakes, $\mathrm{N}_{2}$-fixers cannot keep the system P-limited at low $\mathrm{N}$ inputs because of their higher resource requirements, making them inferior competitors for $\mathrm{P}$ and light. Indeed, competition experiments of cyanobacteria (blue-green algae) show that $\mathrm{N}_{2}$-fixing species are inferior competitors for light as they are competitively excluded by non- $\mathrm{N}_{2}$ fixers when light is limiting (Huisman et al. 1999b; Zevenboom et al. 1981). Additionally, under $\mathrm{N}$-limited conditions, when $\mathrm{N}_{2}$-fixers completely rely on 
$\mathrm{N}_{2}$-fixation for their $\mathrm{N}$ demands, they may become even poorer competitors for light, as demonstrated by markedly increasing $I_{\text {out }}{ }^{*}$ values of a $\mathrm{N}_{2}$-fixing species with decreasing $\mathrm{N}$ supply (Agawin et al. 2007). Furthermore, the observed high $P^{*}$ values of $\mathrm{N}_{2}$-fixing compared to non- $\mathrm{N}_{2}$-fixing cyanobacteria (De Nobel et al. 1997; Passarge et al. 2006), indicate that $\mathrm{N}_{2}$-fixing cyanobacteria are also inferior competitors for $\mathrm{P}$.

A pending question in the debate on the role of $\mathrm{N}$ and $\mathrm{P}$ in ecosystem restoration is whether $\mathrm{N}_{2}$-fixers are capable of correcting ecosystem-scale $\mathrm{N}$ deficiencies, as this would make controlling $\mathrm{N}$ ineffective. Here, we mechanistically demonstrated that $\mathrm{N}_{2}$-fixation is unlikely to completely fulfill the ecosystem's $\mathrm{N}$ demands. As a result, $\mathrm{N}_{2}$-fixation cannot prevent the system from becoming $\mathrm{N}$-limited at low $\mathrm{N}$ input. This is in agreement with the observation that at least half of the freshwater ecosystems on the world are either N-limited or N and P co-limited (Elser et al. 2007). Does this imply that reducing $\mathrm{N}$ inputs to restore aquatic ecosystems would be successful? Our mechanistic models tell us to be careful, as they predict that lowering $\mathrm{N}$ promotes the abundance of $\mathrm{N}_{2}$-fixers that can be just as harmful for the ecosystem as the targeted non- $\mathrm{N}_{2}$-fixing species. Moreover, these $\mathrm{N}_{2}$-fixers provide extra $\mathrm{N}$ to the system and thereby facilitate the targeted $\mathrm{N}$-limited species.

On the other hand, our models probably overestimate the growth potential of $\mathrm{N}_{2}$-fixers by ignoring common ecological, geochemical and energetic constraints on $\mathrm{N}_{2}$-fixation (Berman-Frank et al. 2007; Paerl 1990). For example, our models assume that the $\mathrm{N}_{2}$-fixer's $\mathrm{N}$ demand is immediately fulfilled by $\mathrm{N}_{2}$-fixation and thereby ignore the energetic costs associated with $\mathrm{N}_{2}$-fixation (Paerl 1990). Such constraints could explain that $\mathrm{N}_{2}$-fixation and the associated heterocyst-production was observed to be minimal at low $\mathrm{N}$ supply (Ferber et al. 2004) and that $\mathrm{N}_{2}$-fixers do not always become dominant over non- $\mathrm{N}_{2}$-fixers when reducing $\mathrm{N}$ inputs (Paerl et al. 2014). This is in line with our long-term field data on floating plants, showing that $\mathrm{N}_{2}$-fixers do not necessarily occur at lower $\mathrm{N}$ availability than non- $\mathrm{N}_{2}$-fixers (Fig. 3.3B). From this perspective we argue that controlling $\mathrm{N}$ can be effective given enough constraints on $\mathrm{N}_{2}$-fixation. Hence we advocate a balanced view on ecosystem restoration by not solely focusing on reducing $\mathrm{P}$ inputs but also considering lowering $\mathrm{N}$ inputs (Conley et al. 2009).

Given that excessive nutrient loading is one of the major drivers of global environmental change (Steffen et al. 2015), and that the societal costs associated with mitigating eutrophication are tremendous (Smith et al. 1999), there 
is a clear mandate for the scientific community to come up with an unambiguous standpoint on how to restore aquatic ecosystems. It is often stated that more data, especially from long-term, whole-ecosystem scale studies, is needed to end the existing disagreement (Schindler and Hecky 2009). We here stress the importance of theoretical work, to ensure that the arguments used in the debate are mechanistically sound.

\section{Acknowledgements}

This work was supported by the Netherlands Foundation for Applied Water Research (STOWA) project no. 443237 (to W.M.M and J.J.M.d.K), the USA National Science Foundation INSPIRE Program 1230543 and the Dimensions of Biodiversity Program 1240851 (to H.W.P.). L.P.A.v.G and J.J.M.d.K. conceived and designed the study. All authors discussed the results and contributed to the writing of the manuscript.

\section{Supplementary figures}

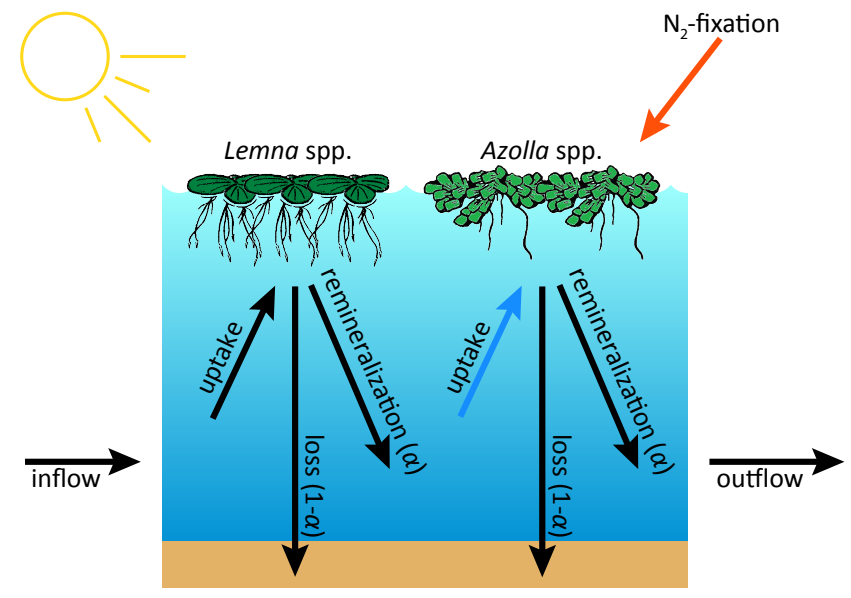

Figure S1. Schematic overview of the resource competition model. The arrows denote the nutrient fluxes; black $=\mathrm{N}$ and $\mathrm{P}$, blue $=\mathrm{P}$, red $=\mathrm{N}$. The non $-\mathrm{N}_{2}$-fixer (Lemna) takes up $\mathrm{N}$ and $\mathrm{P}$ from the water, whereas the $\mathrm{N}_{2}$-fixer (Azolla) takes up only $\mathrm{P}$ from the water and fulfills its $\mathrm{N}$ demand by $\mathrm{N}_{2}$-fixation. When the plants die-off, their nutrient content partly ends up in the water through remineralization $(\alpha)$, and the remaining part $(1-\alpha)$ is lost to the sediment by burial. 


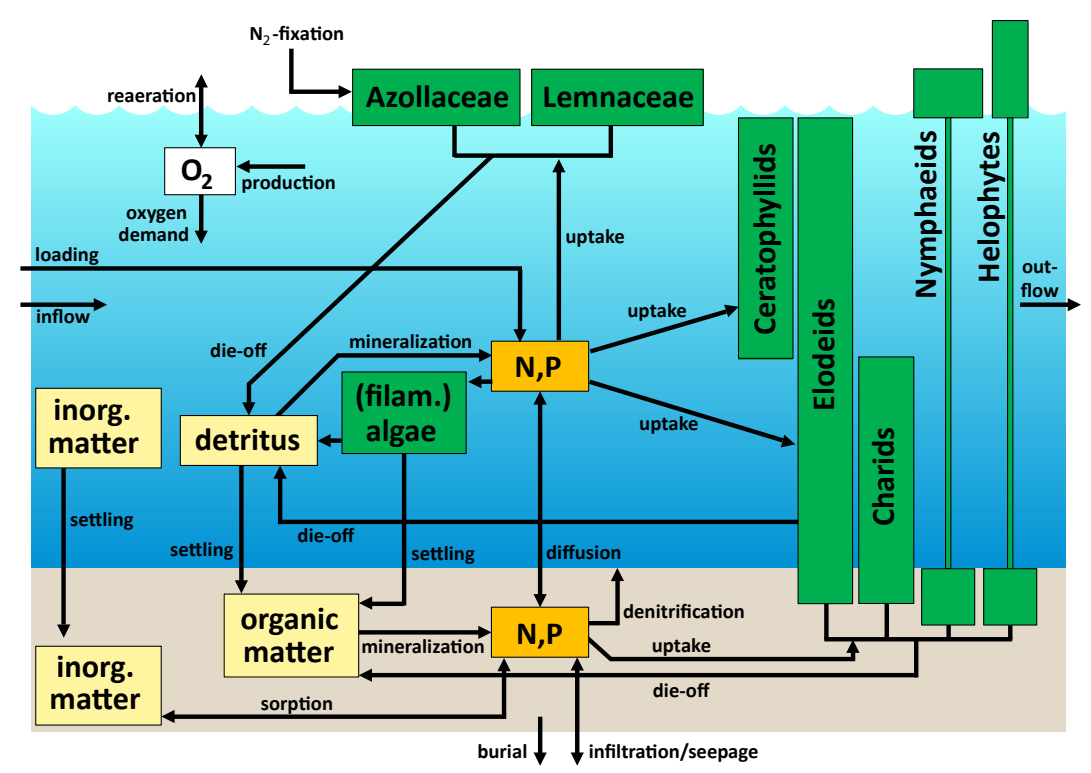

Figure S2. Schematic overview of the complex ecosystem model.
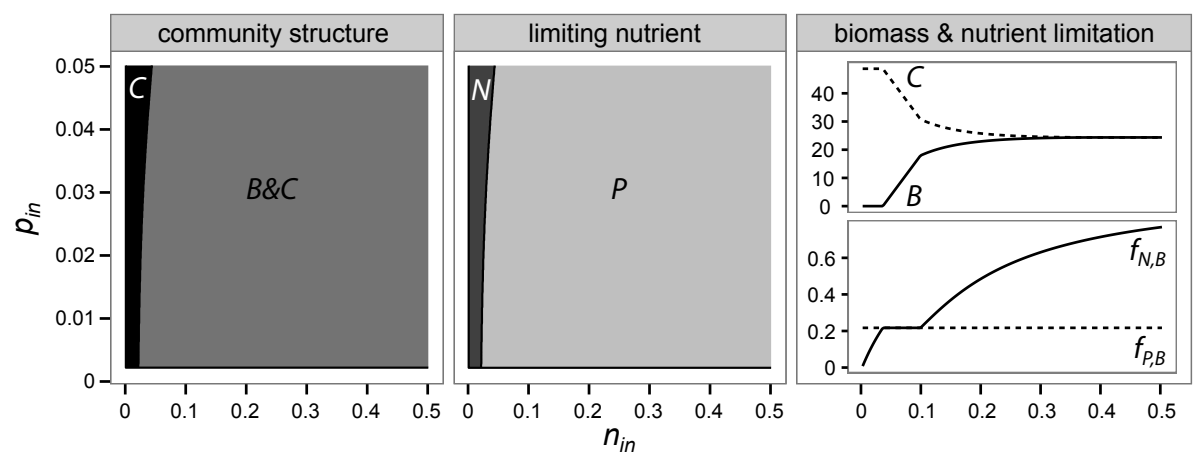

Figure S3. Predicted structure and nutrient limitation of a community of non- $\mathrm{N}_{2}$ fixer $B$ and $\mathrm{N}_{2}$-fixer $C$, for different supply of $\mathrm{N}\left(n_{i n}\right)$ and $\mathrm{P}\left(p_{i n}\right)$, at zero remineralization $(\alpha=0)$. Left panel, competition outcome where ' $\&$ ' denotes coexistence. Middle panel, nutrient that limits the growth of the non- $\mathrm{N}_{2}$-fixer (Eq. 3.5). Right panel, underlying biomasses in $\mathrm{gDW} \mathrm{\textrm {m } ^ { - 2 }}$ and nutrient limitation factors of the non$\mathrm{N}_{2}$-fixer (Eqs. 3.7-3.8) along a gradient of $\mathrm{N}$ supply $\left(p_{i n}=0.04\right)$. Both species were parametrized as Lemna sp. (Table 3.1). 


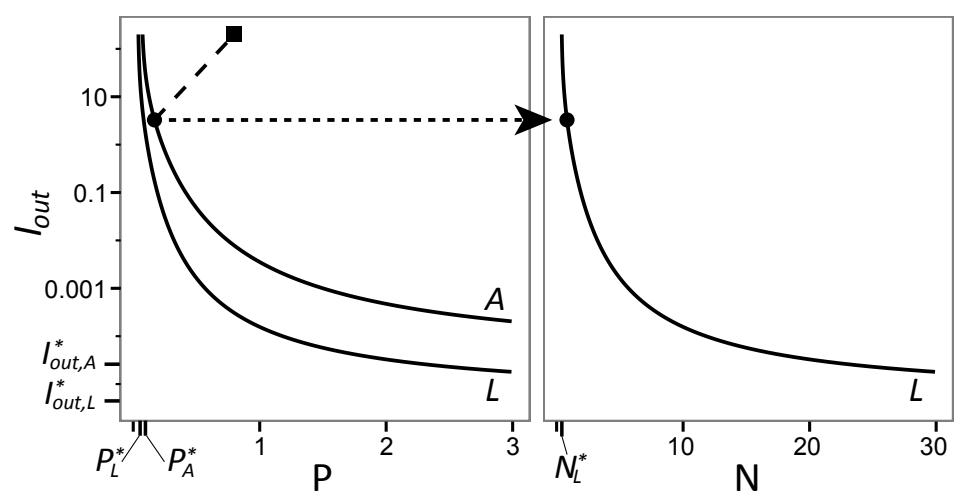

Figure S4. Graphical isocline approach showing why Lemna $(L)$ cannot be P-limited in presence of Azolla $(A)$. The solid lines depict the zero net growth isoclines (ZNGI's) of both species. The ZNGI represents combinations of light intensity at the bottom of the plant $\left(I_{\text {out }}\right)$ and concentrations of $\mathrm{P}$ (left panel) and $\mathrm{N}$ (right panel) in the water at which the net growth of a species is zero, so where its biomass is in equilibrium. The shape of the ZNGI follows from higher nutrient concentrations leading to higher equilibrium biomasses and therefore to more light attenuation and thus a lower $I_{\text {out }}$. The ZNGI captures the competitive ability of a species. The species with the 'lowest' ZNGI is the best competitor because it can deplete the resources to a level too low for the other species to persist. The lowest levels at which Azolla and Lemna can deplete a resource are denoted by $N^{*}$ and $P^{*}$ for nutrients, and $I_{\text {out }}{ }^{*}$ for light. Note that the $N^{*}$ does not apply to Azolla because Azolla does not rely on $\mathrm{N}$ in the water for its growth, as it can fix $\mathrm{N}_{2}$ from the atmosphere and therefore is a superior competitor for N. Lemna, on the other hand, is the best competitor for P and light, due to its lower values of $P^{*}$ and $I_{\text {out }}{ }^{*}$. As a result, Lemna can never be P-limited in presence of Azolla, as Lemna would then lower the availability of light and $\mathrm{P}$ to a level too low for Azolla to persist (left panel). Therefore, if Azolla is present, then Lemna is N-limited. For Azolla to be present, the P:N supply ratio needs to be high enough such that Lemna keeps the P concentration and light availability high enough for Azolla to persist. This is the case for the chosen resource supply in this figure, denoted by the resource supply point (black square; $p_{i n}=0.04, I_{i n}=200$, and $n_{i n}=0.15$ ), indicating the maximum resource availability. The consumption vector (dashed line) shows how the resource availability is depleted till Azolla's biomass reached equilibrium (black dot in left panel). Given the realized light availability (dashed horizontal line), Lemna depletes the $\mathrm{N}$ concentration to a level corresponding with its equilibrium (black dot in right panel). 


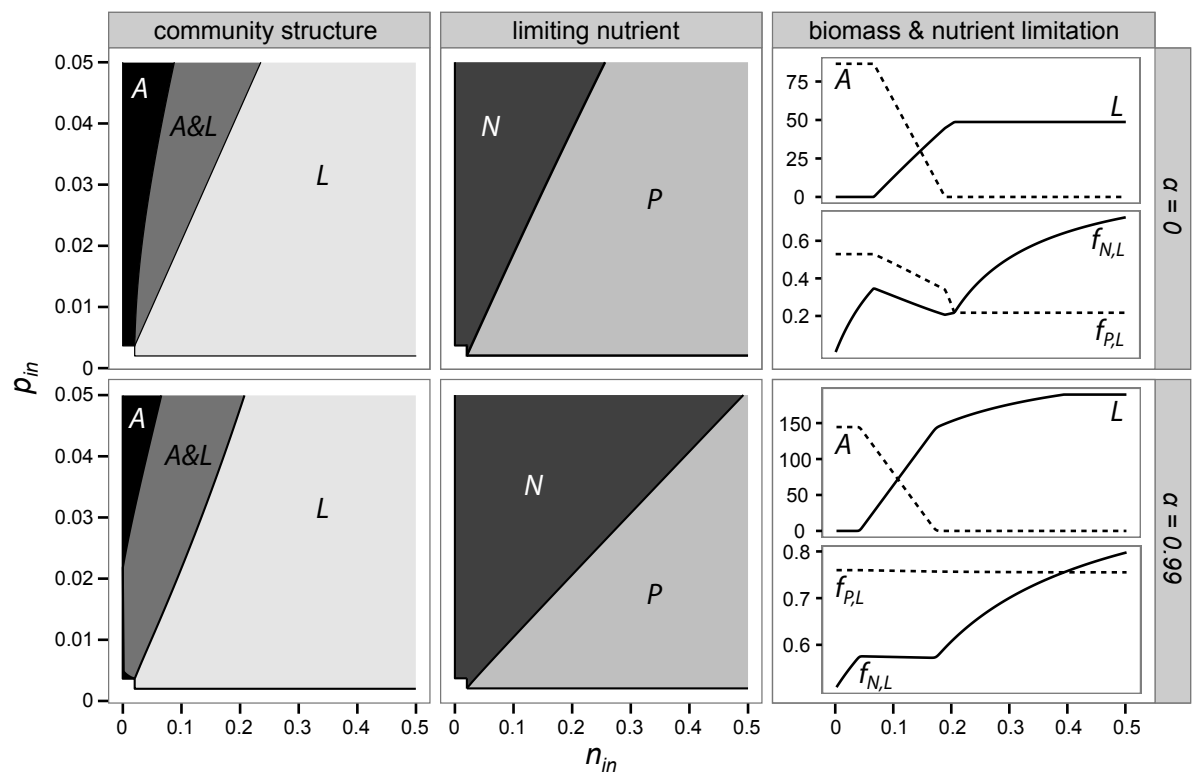

Figure S5. Structure and nutrient limitation of a community of Lemna $(L)$ and Azolla $(A)$, as predicted by the resource competition model at zero mineralization (upper panels: $\alpha=0$ ) and high mineralization (lower panels: $\alpha=0.99$ ). Left panel, competition outcome. Middle panel, nutrient that limits the growth of Lemna. Right panel, underlying biomasses in $\mathrm{gDW} \mathrm{m}^{-2}$ and nutrient limitation factors of Lemna along a gradient of $\mathrm{N}$ supply $\left(p_{i n}=0.04\right)$. See Table 3.1 for parameter values. Note that the nutrient limitation in the $n_{i n}-p_{i n}$ plane at zero remineralization (upper middle panel) is directed by the nutrient uptake and therefore follows (in absence of the $\mathrm{N}_{2}$-fixer) the ratio of the non- $\mathrm{N}_{2}$-fixer's nutrient content $\left(c_{N, L}: c_{P, L} \approx 5\right)$. On the other hand, at high mineralization rates, almost all nutrients are recycled, leading to almost no net uptake, making that nutrient limitation is reflected by the ratio of the $\mathrm{N}: \mathrm{P}$ half-saturation concentrations $\left(M_{N, L}: M_{P, L}=10\right)$. 


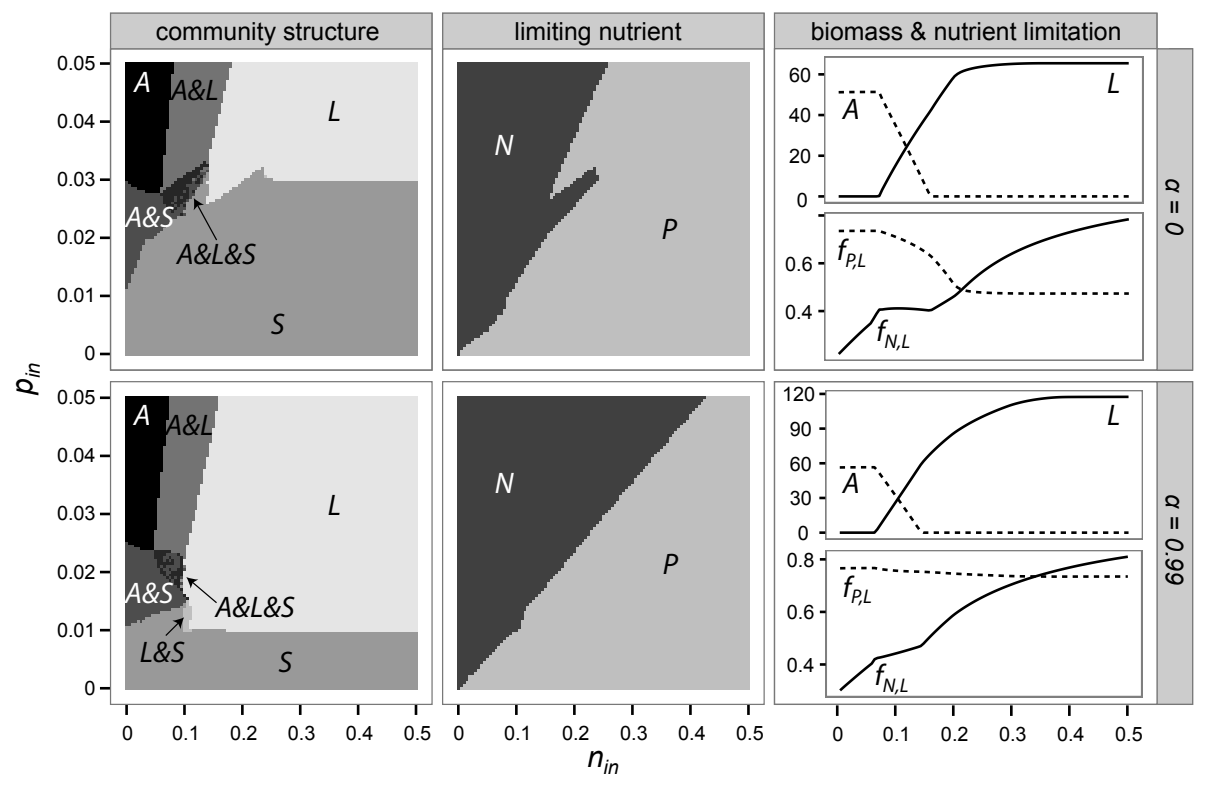

Figure S6. Structure and nutrient limitation of a community of Lemna $(L)$ and Azolla $(A)$, as predicted by the complex ecosystem model at zero mineralization (upper panels: $\alpha=0$ ) and high mineralization (lower panels: $\alpha=0.99$ ). Left panel, competition outcome where 'S' denotes submerged plants. Middle panel, nutrient that limits the growth of Lemna. Right panel, underlying biomasses in gDW m ${ }^{-2}$ and nutrient limitation factors along a gradient of $\mathrm{N}$ supply $\left(p_{i n}=0.04\right)$. See Table 3.1 for parameter values. 



\section{Chapter 4}

\section{How regime shifts in connected aquatic ecosystems are affected by the typical downstream increase of water flow}

van Gerven, L. P. A., J. J. Kuiper, J. H. Janse, A. B. G. Janssen, M. Jeuken, W. M. Mooij and J. J. M. de Klein, 2016. How regime shifts in connected aquatic ecosystems are affected by the typical downstream increase of water flow. Accepted in slightly revised form for publication in Ecosystems.

Abstract. All over the world freshwater ecosystems like ponds, ditches and lakes suffer from nutrient-driven regime shifts to dominance by algae or free-floating plants. Although freshwaters are often connected and part of a network, most of our current knowledge on regime shifts comes from studies of isolated ecosystems. The few studies that did assess the spatial manifestation of regime shifts overlooked the hydrological fact that the water flow through connected waters typically increases in downstream direction. Here, we use a complex ecosystem model to show that the downstream increase in the flow of water, carrying nutrients and biota, does, on its own, not lead to spatial differences in ecology. We support these findings with a simple, analytically tractable, nutrient retention model showing that all connected waterbodies have the same nutrient concentration despite the spatial gradient in water and nutrient flow. Therefore, each connected waterbody is equally vulnerable to a regime shift, implying a regime shift to be systemwide. It appeared that each connected waterbody behaves the same as an isolated waterbody, implying that the vast body of theory on isolated systems, like alternative-stable-states theory, can still be useful for connected systems. Although these findings are violated when there is heterogeneity in lateral runoff or waterbody characteristics - leading to spatial differences in vulne- 
rability - they show that common differences in hydrology not necessarily lead to differences in ecology, and thereby provide a basic concept to better understand the ecology of connected freshwaters.

\subsection{Introduction}

Due to eutrophication, many freshwater ecosystems in the world are subject to catastrophic regime shifts (Scheffer et al. 2001). Such shifts manifest themselves in different ways depending on the type of waterbody. Shallow lake ecosystems may switch from a clear-water macrophyte-dominated state to a turbid-water algae-dominated state, often characterized by toxic cyanobacterial blooms (Jeppesen et al. 1999). Drainage ditches and ponds may switch from a state dominated by submerged plants to a state dominated by freefloating plants such as duckweed, water fern or water hyacinth (Portielje and Roijackers 1995). These free-floating plants frequently cause dark anoxic underwater conditions severely constraining aquatic life and threatening biodiversity (Janes et al. 1996; Verdonschot and Verdonschot 2014).

Over the last decades, ecologists have put considerable effort in understanding and predicting regime shifts. These shifts are often explained from the perspective of alternative stable states (Scheffer et al. 2001). An ecosystem possesses alternative stable states when for the same external conditions the system can be in more than one stable state (Scheffer and Carpenter 2003). This is the case when the system contains positive feedback loops that are strong enough to self-enhance a certain ecosystem state, hampering a transition to a contrasting state (Scheffer et al. 1993). Such a transition can be triggered by changing external conditions, which push the ecosystem towards a threshold or 'tipping point' (Scheffer 1998).

In the case of eutrophication, the threshold level where a regime shift takes place is referred to as the critical nutrient loading (Janse 1997). The critical nutrient loading is not only relevant to systems that possess alternative stable states such as shallow lakes, but also applies to systems that are not likely to have alternative stable states but still can be subject to abrupt shifts, such as ditches (Van Gerven et al. 2015a). The critical nutrient loading of both ditches and shallow lakes was found to depend on system characteristics like water depth, sediment type and water flow (Janse et al. 2008; Van Liere et al. 2007). These characteristics are important because together with the nutrient loading they affect the nutrient concentration in the water and therewith the ecosystem state, as too high nutrient concentrations lead to the dominance 
of free-floating plants or algae (Janse 2005).

Yet, despite the growing insights on regime shifts, it remains largely unclear how regime shifts develop in real ecosystems. A likely reason is that the vast majority of studies on regime shifts, whether empirical or theoretical, have been performed on isolated systems (Ahearn et al. 2013), while it is evident that many natural aquatic ecosystems are in close contact with each other and therefore should be regarded as interdependent systems (Soranno et al. 2010). The few modelling studies that did consider spatial aspects of regime shifts focused mainly on the effects of connectivity through diffusion, and reported profound implications for the manifestation of regime shifts. For example, Bel et al. (2012) and van de Leemput et al. (2015) showed that a regime shift may not be as abrupt as often presumed, but may propagate gradually instead. They also show that diffusion-driven connected ecosystems tend to be in the same state: a local regime shift is either repaired or results in an ecosystem-wide shift. Furthermore, Van Nes and Scheffer (2005) highlight the importance of spatial heterogeneity in environmental characteristics, altering the transient dynamics and allowing for the co-occurrence of alternative stable states. However, these spatial studies did not consider the effects of connectivity through water flow, while aquatic ecosystems are generally part of a catchment, resulting in a hierarchical exchange between systems, from upstream to downstream. The only spatial study we know of that did consider the effect of water flow on regime shifts focused on a chain of lakes (Hilt et al. 2011). This study showed that flushing a chain of lakes leads to remarkably different equilibrium states from upstream to downstream, hampering the occurrence of a complete, system-wide shift.

So far, studies on regime shifts ignored that the water flow through connected aquatic ecosystems typically increases in downstream direction. This increase follows from hydrology. Each system receives water and nutrients by local seepage, precipitation, surface runoff or groundwater leaching (hereafter all together called lateral runoff), which is transported in downstream direction. This gives rise to an increase of water flow from upstream to downstream, in the sense that the most upstream waterbody receives only its own lateral runoff water whereas the most downstream waterbody receives also the accumulated runoff water from all upstream waterbodies. The downstream increase in water flow also leads to a downstream increase of nutrient loading, as the water carries nutrients and to a less extent also life forms. The implication of this spatial gradient in water flow and nutrient loading for the manifestation of regime shifts is yet to be understood. 
In this study we investigate whether the typical downstream increase of water flow in connected aquatic ecosystems affects the vulnerability to regime shifts. Is each system equally vulnerable to a regime shift or does the vulnerability depend on the system's position in the chain or network? To answer this question we follow a stepwise approach with increasing complexity. First, we use a simple nutrient retention model to analyze for a chain of connected water bodies how the build-up of water flow and nutrient loading throughout the chain affects the nutrient concentration. Second, we investigate the consequences for ecology in the same chain of waterbodies, by using the ecosystem model PCDitch, which can predict the regime shift from dominance by submerged plants to free-floating plant dominance (Janse 1998). Third, we investigate the vulnerability to regime shifts in a more complex spatial configuration, a network of ditches, by applying PCDitch coupled to a spatial explicit hydrodynamic model. In the end we discuss on the generality of the found results and their applicability to ponds and lakes. Besides, we validate the results with field observations and elaborate on the implications for the management of connected freshwaters.

\subsection{Methods}

\section{Simple nutrient retention model on a chain of waterbodies}

We used a simple nutrient retention model to describe how the increase of water and nutrient flow throughout a chain of homogeneous well-mixed water bodies affects the nutrient concentration (Fig. 4.1, Table 4.1). The model was adopted from Ahlgren (1980) and we modified it such that the nutrient retention no longer occurs outside the waterbody, but occurs in the waterbody itself, which is, in our view, more realistic.

Each waterbody in the chain has the same water volume $V$, the same nutrient retention processes with rates $r_{0}$ (zero-order process) and $r_{1}$ (first-order process), and the same external nutrient loading $L_{r}$ due to local runoff with discharge $Q_{r}$ and nutrient concentration $C_{r}$. The only aspect that differs is the flow of water between the waterbodies; the most upstream body receives no water from other waterbodies, whereas the most downstream body receives all accumulated runoff water. The accumulation of runoff water results in a downstream increase of water discharge $Q$ and total nutrient loading $L$, where the latter consists of the runoff loading $L_{r}$ and the upstream loading $L_{u}$ associated with the inflow from upstream. 


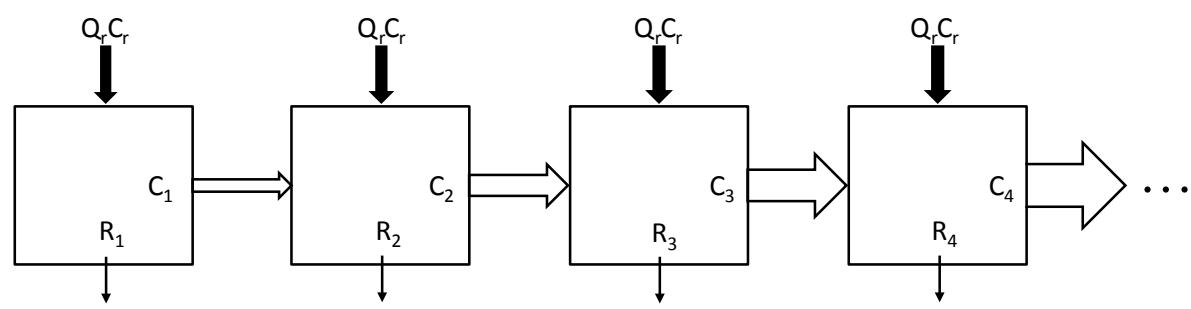

Figure 4.1. Overview of the simple nutrient retention model, predicting the nutrient concentration $C$ in a chain of homogeneous well-mixed waterbodies that each have nutrient retention $R$ and receive water and nutrients by lateral runoff $\left(=Q_{r} C_{r}\right)$, leading to a downstream increase of water and nutrient flow. See Table 4.1 and Eq. 4.1 for symbols and definitions.

Table 4.1. Symbols of the simple nutrient retention model on a chain of waterbodies.

\begin{tabular}{|c|c|c|c|c|}
\hline Symbol & Description & Formula & Unit & Value \\
\hline \multicolumn{5}{|c|}{ State variables: } \\
\hline$C_{i}$ & Nutrient concentration of waterbody $i$ & & $\mathrm{~g} \mathrm{~m}^{-3}$ & \\
\hline \multicolumn{5}{|c|}{ Parameters of each waterbody: } \\
\hline$V$ & Water volume & & $\mathrm{m}^{3}$ & 50 \\
\hline$Q_{r}$ & Discharge from lateral runoff & & $\mathrm{m}^{3} \mathrm{~d}^{-1}$ & 5 \\
\hline$C_{r}$ & Nutrient concentration of lateral runoff water & & $\mathrm{g} \mathrm{m}^{-3}$ & 5 \\
\hline$r_{0}$ & Zero-order nutrient retention rate & & $\mathrm{g} \mathrm{m}^{-3} \mathrm{~d}^{-1}$ & 0.01 \\
\hline$r_{1}$ & First-order nutrient retention rate & & $\mathrm{d}^{-1}$ & 0 to 0.5 \\
\hline \multicolumn{5}{|c|}{ Definitions: } \\
\hline$L_{r}$ & Waterbody's nutrient loading from lateral runoff & $L_{r}=Q_{r} C_{r}$ & $\mathrm{~g} \mathrm{~d}^{-1}$ & \\
\hline$L_{u, i}$ & Upstream nutrient loading of waterbody $i$ & $L_{u, i}=(i-1) Q_{r} C_{i-1}$ & $\mathrm{~g} \mathrm{~d}^{-1}$ & \\
\hline$Q_{i}$ & Total discharge of waterbody $i$ & $Q_{i}=i Q_{r}$ & $\mathrm{~m}^{3} \mathrm{~d}^{-1}$ & \\
\hline$R_{i}$ & Nutrient retention of waterbody $i$ & $R_{i}=r_{0} V+r_{1} V C_{i}$ & $\mathrm{~g} \mathrm{~d}^{-1}$ & \\
\hline
\end{tabular}

The dynamics of the nutrient concentration $C$ of waterbody $i$ then read

$$
V \frac{\mathrm{d} C_{i}}{\mathrm{~d} t}=Q_{r} C_{r}+(i-1) Q_{r} C_{i-1}-i Q_{r} C_{i}-r_{0} V-r_{1} V C_{i}
$$

and depend on the lateral runoff loading $L_{r}$ (first term at the right hand side), the upstream loading $L_{u}$ (second term), the nutrient outflow (third term) and the nutrient retention $R$ (fourth and fifth term). We determined the waterbodies' final nutrient concentrations by deriving them analytically from Eq. 4.1 as well as by running the model till equilibrium, using the parameter values given by Table 4.1. The model was run in R (R Core Team 2013), using the deSolve Package (Soetaert et al. 2010). 


\section{Complex ecosystem model on a chain of ditches}

We analyzed the same chain of homogenous waterbodies for its ecological state by using PCDitch, an ecosystem model for ditches (Janse 1998). Due to the importance of water plants for the ecological functioning of ditches, PCDitch has a strong focus on macrophytes. It simulates the competition of six different water plant groups and one group of algae for nitrogen $(\mathrm{N})$, phosphorus (P) and light (Fig. 4.2). To do so, it keeps track of the cycling of $\mathrm{N}, \mathrm{P}$ and oxygen in the water column and the sediment layer. The model calculates the resulting daily macrophyte abundance, given the imposed water temperature, light intensity, nutrient loading and water flow. As an emergent property, PCDitch can predict the regime shift from dominance of submerged vegetation (parametrized as Elodea spp.) to free-floating plants (parametrized as Lemna spp.) (Van Liere et al. 2007).

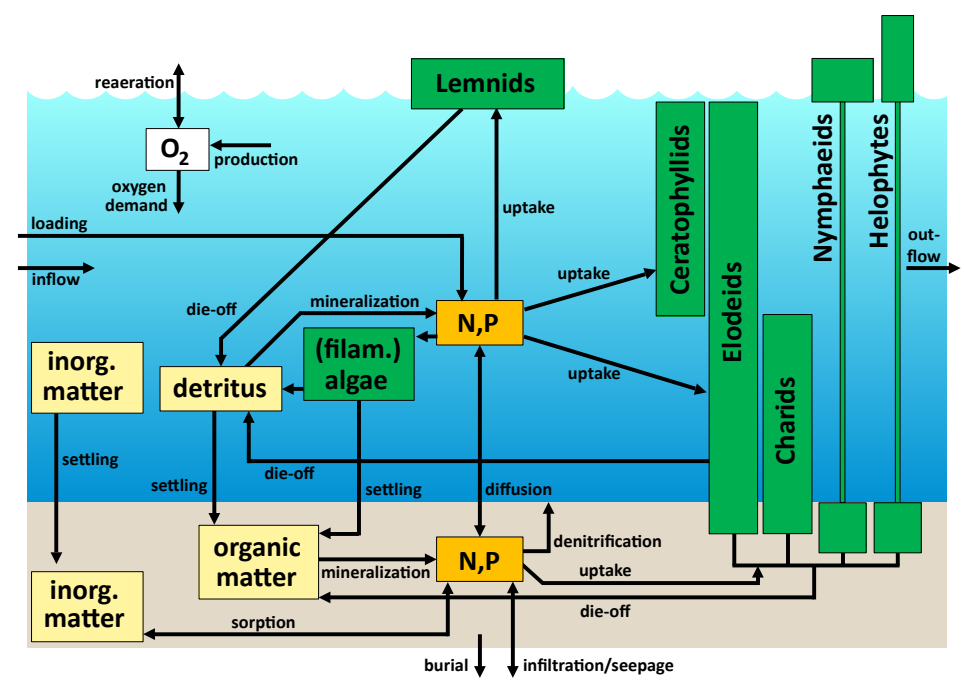

Figure 4.2. Components and processes of the ecosystem model PCDitch, after Janse (1998).

To calculate the ecological state in the chain, we ran PCDitch (in R) sequentially from upstream to downstream by using the outflow of one ditch as the inflow for the next ditch. We ran PCDitch till seasonal equilibrium (model results that repeat themselves every year) was reached, which took about 20 years in the model. For the process parameters, water temperature and light intensity, we used the settings of the calibrated model (Janse 1998), resembling an 'average ditch' in the Netherlands. For the external input of 
water and nutrients we assumed that each ditch in the chain had the same seasonal amount of lateral runoff, for which we took the average seasonal amount in Dutch polders (Fig. 4.3). We converted this amount from a loading per $\mathrm{m}^{2}$ runoff area (land) to a loading per $\mathrm{m}^{2}$ ditch by assuming a ratio of ditch to land area of 0.023 (Schultz 1992). We assigned each ditch a water depth of $0.5 \mathrm{~m}$ and an area of $100 \mathrm{~m}^{2}$, leading to a water volume of $50 \mathrm{~m}^{3}$, which equals the volume used in the simple nutrient retention model.

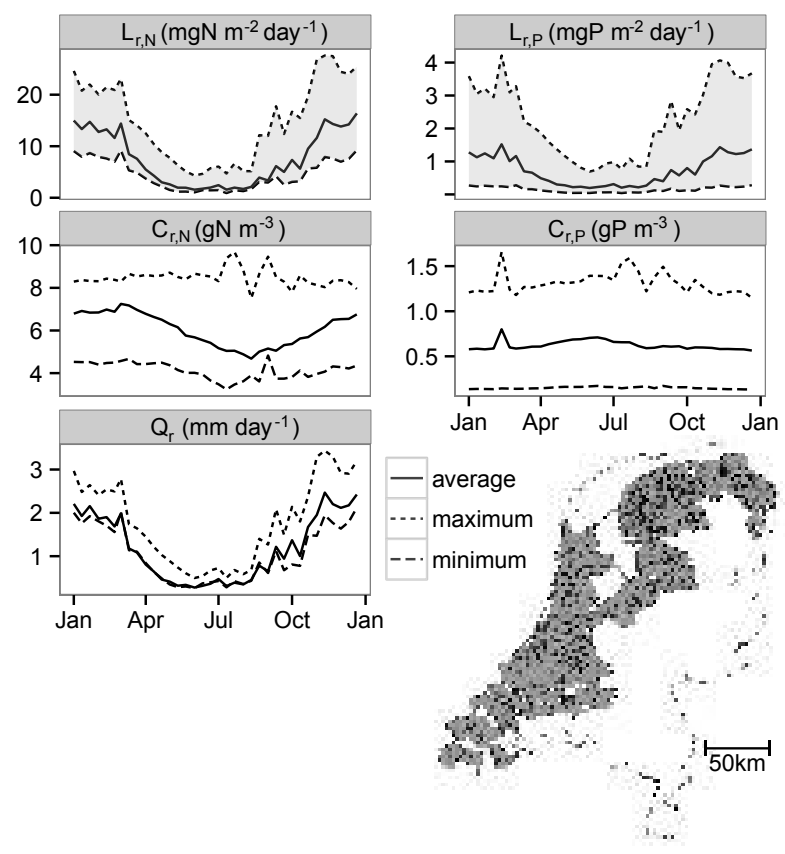

Figure 4.3. Average, maximum and minimum seasonal lateral runoff in 156 Dutch polders (see map) from 1990 to 2010, calculated with the STONE model (Wolf et al. 2005). The runoff nutrient loading $L_{r}$ (upper panels), expressed per $\mathrm{m}^{2}$ runoff area, results from the runoff concentration $C_{r}$ (middle panels) multiplied by the runoff discharge $Q_{r}$ (lower panel).

\section{Complex ecosystem model on a network of ditches}

We used PCDitch also to analyze the vulnerability to regime shifts in a more complex spatial configuration: a network of ditches. We chose a network that resembles a typical ditch network in Dutch polder systems; a rectangular network in which parallel $1000 \mathrm{~m}$-long secondary ditches, with a distance 
of $50 \mathrm{~m}$ between them, cross 3 primary $850 \mathrm{~m}$-long ditches every $500 \mathrm{~m}$. The runoff water accumulates in the direction of the polder outlet, located at the end of one of the primary ditches, where it is pumped into the higher-situated nearby river. We assigned each ditch a water depth of $0.5 \mathrm{~m}$ and a width of 1 $\mathrm{m}$. These dimensions are common for Dutch polder ditches (Schultz 1992).

We applied the 1-D hydrodynamic model SOBEK (Delft Hydraulics 1996) to calculate the water flow in the We used the 1-D hydrodynamic model SOBEK (Delft Hydraulics 1996) to calculate the water flow in the network. This water flow served as input to calculate the ecological state of each ditch with PCDitch. To establish this coupling of PCDitch with SOBEK, the equations of PCDitch were implemented in DELWAQ (Delft Hydraulics 1995), which took care of the information exchange between SOBEK and PCDitch, and calculated the resulting flow of substances (e.g. nutrients) in the network. To implement PCDitch in DELWAQ, we used a database approach to modelling (DATM) as presented by Mooij et al. (2014), which facilitates the implementation of a model in a new modelling environment (Van Gerven et al. 2015b). We verified that the coupling was successful by performing benchmark runs and found that numerical dispersion did not affect the PCDitch-SOBEK results, since decreasing the default calculation time step of 10 minutes did not alter the model results.

To calculate the ecological state of the network, we ran PCDitch-SOBEK till seasonal equilibrium was reached, which took about 50 years in the model. We assigned the same lateral runoff to each meter of ditch length, again using the average seasonal runoff in Dutch polders (Fig. 4.3). To simulate a regime shift from dominance of submerged plants (Elodea spp.) to free-floating plants (Lemna spp.), we reran PCDitch-SOBEK while increasing the external nutrient input, by raising the runoff nutrient concentration $C_{r}(t)$.

Finally, we repeated all calculations, but now with spatial variations in ditch characteristics and external nutrient input. The ditch characteristics were varied by doubling the width of the primary ditches, as these ditches are in general wider because they discharge more water. The width of the secondary ditches was kept the same. The lateral nutrient input was varied by randomly changing the nutrient concentration of lateral runoff $C_{r}(t)$ of each ditch within plus or minus $30 \%$ of its original value, mimicking the natural variations of lateral runoff in Dutch polders (Oenema et al. 2005). 


\subsection{Results}

\section{Nutrient concentrations in a chain of waterbodies}

The simple nutrient retention model predicts for a chain of waterbodies that the typical downstream increase of water and nutrient flow does, on its own, not lead to different nutrient concentrations in the chain (Fig. 4.4). Instead, each waterbody eventually has the same nutrient concentration. This is a rather counterintuitive result, given the downstream increase in discharge and nutrient loading, and the associated spatial gradient in the average concentration of the incoming water (Fig. 4.4).
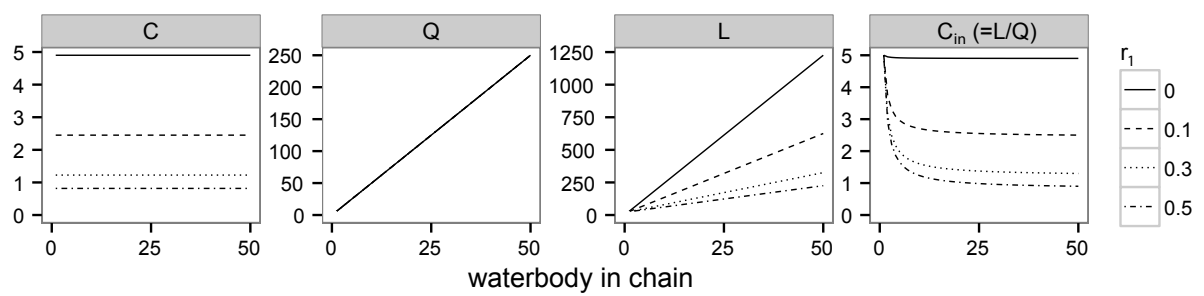

Figure 4.4. Results of the simple nutrient model for different nutrient retention rates $r_{1}\left(\mathrm{~d}^{-1}\right)$, showing that each waterbody in the chain has the same equilibrium nutrient concentration $C\left(\mathrm{~g} \mathrm{~m}^{-3}\right)$ despite a downstream increase of discharge $Q\left(\mathrm{~m}^{3} \mathrm{~d}^{-1}\right)$ and total nutrient loading $L\left(\mathrm{~g} \mathrm{~d}^{-1}\right)$, as well as a downstream decrease of the average concentration of the incoming water $C_{i n}\left(\mathrm{~g} \mathrm{~m}^{-3}\right)$.

This result can be understood by regarding the analytic solution of the equilibrium concentration $C^{*}$ of waterbody $i$, which can be derived from Eq. 4.1 (Appendix A):

$$
C_{i}^{*}=\frac{Q_{r} C_{r}-r_{0} V}{Q_{r}+r_{1} V} .
$$

Eq. 4.2 shows that the equilibrium concentration depends only on the waterbody's local properties (the lateral input of water and nutrients $\left(Q_{r}\right.$ and $\left.C_{r}\right)$, water volume $(V)$ and nutrient retention rates $\left(r_{0}\right.$ and $\left.\left.r_{1}\right)\right)$, which are the same for each waterbody, explaining the uniform nutrient concentration in the chain. Apparently, the equilibrium concentration does not depend on water and nutrient fluxes from upstream, implying that each connected waterbody in fact behaves the same as an isolated waterbody that receives water and nutrients from lateral runoff only. 


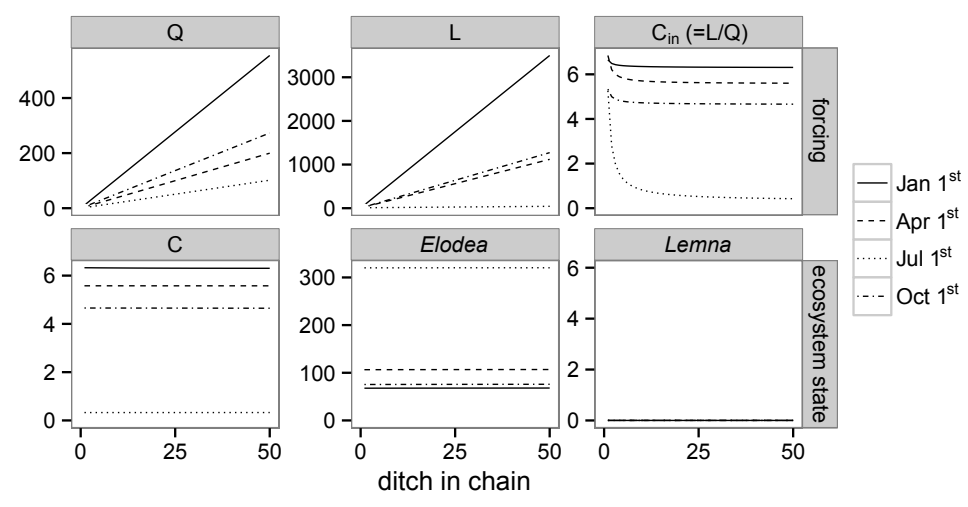

Figure 4.5. Results of the complex ecosystem model for different days of the year, showing that each ditch in the chain has the same equilibrium state (lower panels) indicated here by the nitrogen concentration $C\left(\mathrm{gN} \mathrm{m}^{-3}\right)$ and the plant dry weight $(\mathrm{g}$ $\mathrm{m}^{-2}$ ) of waterweed (Elodea) and duckweed (Lemna) - despite spatial difference in the forcing variables (upper panels): discharge $Q\left(\mathrm{~m}^{3} \mathrm{~d}^{-1}\right)$, total nitrogen loading $L$ (gN $\left.\mathrm{d}^{-1}\right)$ and average concentration of the incoming water $C_{i n}\left(\mathrm{gN} \mathrm{m}^{-3}\right)$.

\section{Ecological state in a chain of ditches}

The complex ecosystem model PCDitch predicts that the downstream increase of water and nutrient flow in the chain not only leads to the same nutrient concentration throughout the chain, but also to the same ecological state, as indicated by the uniform abundance of submerged plants (Elodea spp.) and floating plants (Lemna spp.) (Fig. 4.5). Note that this uniform ecological state changes over time due to seasonality of the imposed conditions (temperature, light intensity and runoff). For the used settings, the waterbodies in the chain are dominated by submerged plants, at the cost of floating plants.

\section{Ecological state and regime shifts in a network of ditches}

Even in a more complex spatial configuration, such as a network of ditches, the downstream increase of water and nutrient flow does not lead to spatial differences in the ecological state, as predicted by PCDitch coupled to the 1-D hydrodynamic model SOBEK. As a result, each waterbody in the network is equally vulnerable to a regime shift. Therefore, raising the nutrient loading by lateral runoff $L_{r}$ leads to a system-wide regime shift, as all ditches in the network switch from dominance of submerged to floating plants at the same time (Fig. 4.6A). 

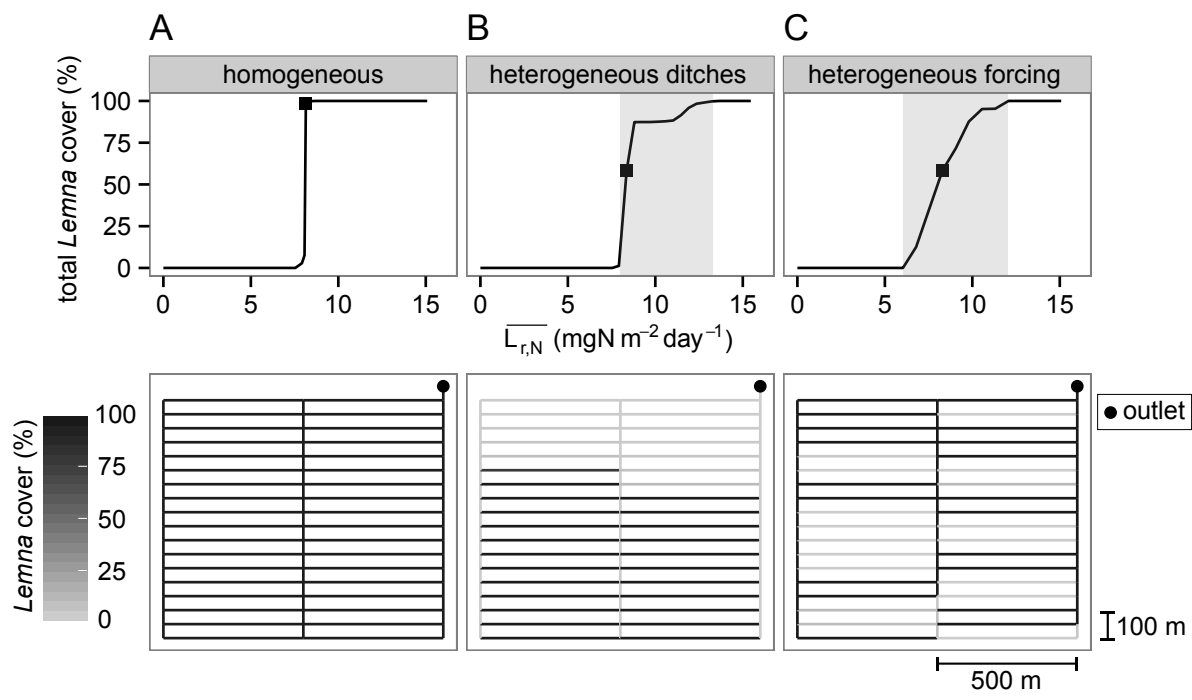

Figure 4.6. Results of applying the complex ecosystem model to a network of ditches showing how the network-wide summer-averaged floating-plant cover (Lemna) (upper panels) depends on the yearly averaged $\mathrm{N}$ loading by lateral runoff $\overline{L_{r, N}}$ (per $\mathrm{m}^{2}$ runoff area), in the case of (A) homogeneous ditches with homogeneous lateral runoff, (B) heterogeneous ditches (wider primary ditches) and (C) heterogeneous lateral runoff (runoff concentration to a ditch is randomly varied within $\pm 30 \%$ ). The grey areas indicate the network-wide range of critical nutrient loadings. The lower panels show the network's Lemna coverage for the runoff loadings indicated by the black squares.

However, the ditches do have a different vulnerability to a regime shift when accounting for spatial variations in ditch characteristics (wider primary ditches; Fig. 4.6B) or in nutrient loading by runoff (randomly varied runoff; Fig. 4.6C). Then, a regime shift is not system-wide anymore, as each ditch switches to floating-plant dominance at a different moment when raising the nutrient loading $L_{r}$ by lateral runoff. In addition, the vulnerability to a regime shift now depends on the position of the waterbody in the network. This is illustrated by the case of the widened primary ditches in which the vulnerability to floating-plant dominance not only changes for the widened ditches - it decreases because of a dilution effect - but also changes for the non-widened secondary ditches (Fig. 4.6B). Especially the non-widened ditches close to the polder outlet become less vulnerable, as they receive more water from the primary ditches and therefore become more diluted. 


\subsection{Discussion}

\section{Uniform regime shifts in connected freshwater ecosystems}

Our analyses show that the typical downstream increase of water flow in connected waters does not automatically lead to differences in the vulnerability to a regime shift. Instead, we found that all systems in a chain or network are equally vulnerable to a regime shift, implying a regime shift to be system-wide. This is the case when looking solely at the effect of increasing water flow, in the sense that the characteristics of all waterbodies (such as water depth and sediment type) are the same and that the increase in water flow is gradual because each waterbody has the same lateral input of water and nutrients by runoff.

We used a simple nutrient retention model to better understand this equal vulnerability. This model describes the nutrient concentration in a chain of waterbodies, as the nutrient concentration is of great importance for the ecological state and thus for the chance on a regime shift. The model shows that the gradual downstream increase of water and nutrient flow leads to the same nutrient concentration throughout the chain. Apparently, the downstream decrease in the concentration of the incoming water $C_{i n}$ (Fig. 4.4) is balanced by the smaller effect of nutrient retention in downstream direction due to the shorter water residence times, such that the final nutrient concentration is the same from upstream to downstream. The similar nutrient concentrations can also be explained by realizing that the concentration of a waterbody does not change when the waterbody is flushed with water from an upstream waterbody of the same concentration, as is the case in our configuration. This also explains why solving the model equations for equilibrium (Eq. 4.2) shows that each connected waterbody basically behaves the same as an isolated waterbody that receives no water and nutrients from upstream but only from lateral runoff.

\section{Applicability of the found results to other ecosystems}

Although in this study we focused on the vulnerability to regime shifts in connected ditches, we expect that our findings also apply to other connected ecosystems like chains or networks of ponds and lakes, also because our findings are explained by the simple nutrient retention model that applies to any waterbody. To be sure, we applied the shallow-lake ecosystem model PCLake (Janse et al. 2010) to a chain of lakes and found similar results (not shown here): also in a chain of lakes each waterbody can be in the same ecological 
state and therefore has the same vulnerability to a regime shift, despite the downstream increase of water flow. It concerns a regime shift from a clear lake dominated by submerged vegetation to a turbid lake dominated by algae. Even the fact that these algae are transported by the water does not lead to spatial differences in the vulnerability to a regime shift. Furthermore, we checked that the lakes are also equally vulnerable to the backward shift from turbid to clear. Note that this shift occurs at a much lower external nutrient input by runoff because the lakes possess alternative stable states.

In fact, we expect that any dynamic process-based model that incorporates water, nutrients and biota can predict that the downstream increase of water flow in connected systems can lead to a uniform ecological state, provided that each waterbody has the same process rates, the same characteristics and the same local forcings (e.g. lateral input of water and nutrients, temperature, light). Even the size of the waterbodies may differ as long as the local process rates, characteristics and forcings are the same per $\mathrm{m}^{2}$ of waterbody surface.

\section{Validity of the found results}

Our main result - that waterbodies in a connected system are equally vulnerable to a regime shift despite the downstream increase of water flow - only holds true when regarding the simplest conditions. When accounting for spatial heterogeneity in waterbody width or lateral nutrient input by runoff, we found that each connected waterbody gets a different vulnerability, which also depends on the position of the waterbody in the chain or network. We found similar results when we varied waterbody characteristics other than water width, such as water depth and sediment type. The same holds when spatially varying environmental conditions other than lateral nutrient input, such as light intensity or temperature.

Furthermore, differences in vulnerability also originate from ignoring lateral runoff. The runoff water then no longer accumulates in downstream direction, implying that the water flow does not increase anymore but remains constant. The model of Hilt et al. (2011) predicted that flushing a chain of lakes with such a constant water flow leads to profound spatial differences in ecosystem state. The upstream lakes appeared to be more vulnerable to a regime shift from a clear state to a turbid state than the downstream lakes. We expect that our models would predict a similar downstream improvement of the ecosystem state, as the nutrient concentration of the flushing water is likely to decrease in downstream direction due to nutrient retention. In contrast, lake chains often show an upstream improvement in ecosystem 
state (Fisher et al. 2009; Hillbricht-Ilkowska 2002; Soranno et al. 1999). Hilt et al. (2011) therefore acknowledged that a more realistic prediction requires the consideration of lateral inputs of water and nutrients, as we did in this study.

\section{Comparison with field observations}

Based on our findings, one would expect that connected freshwaters located in a fairly homogeneous landscape (in terms of its waterbody characteristics as well as its land use and soil type enabling homogeneous runoff) have a similar ecosystem state. Indeed, field data on 9 North-American lake chains show a high synchrony in ecosystem state, especially for lakes with short water residence times (Soranno et al. 1999). On the other hand, other field studies on seemingly homogeneous landscapes show that ponds connected through overflows show distinct ecosystem states and even a co-occurrence of different states (Cottenie et al. 2003; Van Geest et al. 2003). However, such a co-occurrence of states may be temporal, like in the Dutch Lake Veluwe where the co-occurrence of a clear water with vegetation and turbid water with algae (Scheffer et al. 1994) turned out to be a transition phase of the whole lake to a clear-water state (van de Leemput et al. 2015). Furthermore, in line with our findings, field studies showed that environmental variability can lead to differences in ecosystem state. For example, landscape properties were found to be one of the main causes of the considerable differences in macrophyte vegetation in 50 connected boreal lakes (Mäkelä et al. 2004). In addition, we found that due to environmental variability the position of a waterbody in a chain or network becomes important. This is supported by a study on 71 lakes in Michigan USA, whose variation in ecosystem state could be largely explained by the position of the lake in the landscape (Martin and Soranno 2006). Furthermore, Carpenter and Lathrop (2014) point at the importance of inter-annual variation in runoff to explain variability in ecosystem state, and illustrated this for 4 lakes of the Yahara chain (Wisconsin, USA).

\section{Implications for management of connected freshwaters}

Globally much effort is put into the restoration of eutrophic aquatic ecosystems (Sondergaard et al. 2007). Nutrient reduction is generally considered to be the most effective restoration measure. Knowing the critical nutrient loading at which the system shows a swift recovery to a desired state provides managers with a clear target that can easily be communicated (Jaarsma et al. 2008). However, so far it is not clear yet how the concept of critical loading should be applied to connected systems. 
Based on our results, the regime shift of connected waters in fairly homogenous landscapes can already be predicted by regarding the nutrient loading from lateral runoff only. The other part of the nutrient loading, from upstream waterbodies, may be ignored, as we found that the connected waterbodies tend to behave the same as an isolated waterbody that receives no water and nutrients from upstream but only from lateral runoff. Therefore, the critical nutrient loading can already be estimated with a non-spatial ecosystem model. This approach does not apply to more heterogeneous landscapes. Then, each waterbody has a different vulnerability to a regime shift and also has a different critical nutrient loading. As the vulnerability now depends on the position of the waterbody in the chain or network, the nutrient loading from upstream can no longer be ignored. Therefore, the prediction of the critical nutrient loading becomes much more difficult and requires a spatial modeling approach in which ecology and hydrology are integrated.

\section{Conclusions}

In this study we regard connected aquatic ecosystems in their hydrological context, as part of a watershed. We show that the associated downstream increase of water flow, on its own, does not lead to spatial variations in ecology. As a result, all connected waterbodies are equally vulnerable to a regime shift, implying a regime shift to be system-wide. All connected waterbodies turned out to behave the same as an isolated waterbody, implying that the vast body of existing theory on isolated systems can still be of use for connected systems. Although these findings are violated when there is spatial heterogeneity in lateral runoff or waterbody characteristics - each connected waterbody then has a different vulnerability to a regime shift - they constitute a basic concept on how hydrology affects the ecology of connected freshwaters.

\section{Acknowledgements}

We thank Martin Mulder, Erwin van Boekel and Piet Groenendijk for providing runoff data. Furthermore, we thank Sven Teurlincx, Kristin Schulz and Michiel Verhofstad for the fruitful discussions on this paper. This work is financed by the Netherlands Foundation for Applied Water Research (STOWA) project no. 443237 and by the Netherlands Organisation for Scientific Research (NWO) project no. 842.00.009. This is publication 6151 of the Netherlands Institute of Ecology (NIOO-KNAW). 


\section{Appendix A: Derivation of the equilibrium concentration in a chain of waterbodies}

Here we derive the equilibrium nutrient concentration in the chain of waterbodies, following from the simple nutrient retention model (see Eq. 4.1). First, we deduce the equilibrium concentration of the first waterbody $C_{1}^{*}$ by solving for $\mathrm{d} C_{1} / \mathrm{d} t=0$, which gives

$$
\frac{Q_{r}}{V}\left(C_{r}-C_{1}^{*}\right)-r_{0}-r_{1} C_{1}^{*}=0 \Leftrightarrow C_{1}^{*}=\frac{Q_{r} C_{r}-r_{0} V}{Q_{r}+r_{1} V} .
$$

Similarly, $C_{2}^{*}$ can be derived:

$$
\frac{Q_{r}}{V}\left(C_{r}+C_{1}^{*}-2 Q_{r} C_{2}^{*}\right)-r_{0}-r_{1} C_{2}^{*}=0 \Leftrightarrow C_{2}^{*}=\frac{Q_{r} C_{r}+Q_{r} C_{1}^{*}-r_{0} V}{2 Q_{r}+r_{1} V} .
$$

Substituting $C_{1}^{*}$ (Eq. A.1) in the expression for $C_{2}^{*}$ (Eq. A.2), and simplifying the resulting expression (with Mathematica) leads to

$$
C_{2}^{*}=\frac{Q_{r} C_{r}-r_{0} V}{Q_{r}+r_{1} V} .
$$

Note that this final expression for $C_{2}^{*}$ equals the expression for $C_{1}^{*}$ (Eq. A.1). The same expression can be found for waterbodies further in the chain, again by deriving their equilibrium concentration $C_{i}^{*}$ and substituting the equilibrium concentration of the upstream waterbody $C_{i-1}^{*}$ :

$$
C_{i}^{*}=\frac{Q_{r} C_{r}+(i-1) Q_{r} C_{i-1}^{*}-r_{0} V}{i Q_{r}+r_{1} V}=\frac{Q_{r} C_{r}-r_{0} V}{Q_{r}+r_{1} V} .
$$

Eq. A.4 shows that each waterbody in the chain has the same equilibrium concentration. 


\section{Chapter 5}

\section{Advantages of concurrent use of multi- ple software frameworks in water qual- ity modelling using a database approach}

van Gerven, L. P. A., R. J. Brederveld, J. J. M. de Klein, D. L. DeAngelis, A. S. Downing, M. Faber, D. J. Gerla, Jochem /textquotesingle t Hoen, J. H. Janse, A. B. G. Janssen, M. Jeuken, B. W. Kooi, J. J. Kuiper, B. Lischke, S. Liu, T. Petzoldt, S. A. Schep, S. Teurlincx, C. Thiange, D. Trolle, E. H. van Nes, W. M. Mooij 2015. Advantages of concurrent use of multiple software frameworks in water quality modelling using a database approach. Fundamental and Applied Limnology 185: 5-20.

Abstract. Water quality modelling deals with multidisciplinary questions ranging from fundamental to applied. Addressing this broad range of questions requires multiple analysis techniques and therefore multiple frameworks. Through the recently developed database approach to modelling (DATM), it has become possible to run a model in multiple software frameworks without much overhead. Here we apply DATM to the ecosystem model for ditches PCDitch and its twin model for shallow lakes PCLake. Using DATM, we run these models in six frameworks (ACSL, DELWAQ, DUFLOW, GRIND for MATLAB, OSIRIS and R), and report on the possible model analyses with tools provided by each framework. We conclude that the dynamic link between frameworks and models resulting from DATM has the following main advantages: it allows one to use the framework one is familiar with for most model analyses and eases switching between frameworks for complementary model analyses, including the switch between a 0-D and 1-D to 3-D setting. Moreover, the strength of each framework - including runtime performance - can now be easily exploited. We envision that a community-based further 
development of the concept can contribute to the future development of water quality modelling, not only by addressing multidisciplinary questions but also by facilitating the exchange of models and process formulations within the community of water quality modellers.

\subsection{Introduction}

Water quality modelling often deals with multidisciplinary issues ranging from fundamental questions aiming at a more thorough understanding of theoretical principles to applied questions like the scenario-wise evaluation of potential measures for ecosystem management. This diversity in questions requires a multitude of model analysis techniques and therefore a multitude of software frameworks, as there is no single framework that captures all these techniques. Ideally, one would like to easily implement a model in a framework of choice and easily switch between existing frameworks to exploit the myriad of available analysis techniques.

The number of software frameworks that is available to implement water quality models is large and still increasing (Argent 2004). This makes it nearly impossible to have an overview of existing frameworks and their capabilities. As a result, experienced users stick to the framework they have invested in, instead of exploiting the rich array of choices that exists. At the same time, new users choose the framework they have easiest access to, and for which they can get support from experienced users in their direct vicinity.

Switching between frameworks currently takes considerable effort as models are often locked in a single framework, in that they are written in framework-specific code and can only be accessed through framework-specific user interfaces (David et al. 2013). This phenomenon is also referred to as framework invasiveness (Lloyd et al. 2011). This harbours the risk that framework familiarity tends to define which model to use, instead of the ecological question that needs to be answered (Argent 2004). The observed multitude of frameworks and their locked-in models leads us to conclude that the landscape of water quality modelling is highly fragmented. This fragmentation often leads to a 'reinvention of the wheel' and 'tunnel-vision' in water quality modelling, as there is no healthy cross-fertilization of ideas between models and frameworks (Mooij et al. 2010).

A database approach to modelling (DATM) was recently proposed to address this challenge (Mooij et al. 2014). In this approach, the knowledge incorporated in a model is stored in a database, independently of program 
language and framework. In order to run the model in a certain framework, the information in the database is translated and augmented with language and framework specifics. This process is automated so that the model can easily be re-implemented in the framework after it has been modified in the database. Thus, with DATM it becomes easy to switch between multiple frameworks and exploit their joint multitude of model analysis techniques to address the multidisciplinary questions such as encountered in water quality modelling.

Here we apply DATM to analyse the ecosystem model for ditches PCDitch (Janse 1998) and its twin model for shallow lakes PCLake (Janse 1997) in six different software frameworks, including non-spatial and 1-D to 3-D implementations of the models. After determining the runtime of the models in the different frameworks, we analysed both models and report on the used framework tools for sensitivity analysis, calibration, validation, uncertainty analysis, bifurcation analysis and scenario analysis. We discuss the benefits and potential pitfalls of using DATM in water quality modelling with respect to exploiting the complementarity and redundancy among frameworks. Additionally, we discuss the possibilities it creates for model and framework review. Because DATM relies on mathematics, we conclude that it can effectively be used for a much wider range of models and frameworks than studied here, and may contribute to the future development of water quality modelling.

\subsection{Methods}

\section{Framework-implementation of the models}

We used DATM to implement PCDitch and PCLake in six different frameworks. In this process, the ordinary differential equations (ODE's) of the models are translated into framework-specific code (Mooij et al. 2014). The complete implementation process - from building the translators to performing test runs - is described in detail in Appendix A.

Using DATM, ODE-based models - describing the change of a state variable in time - can also be implemented in a spatial setting. For example, we implemented PCDitch and PCLake in the frameworks DUFLOW and DELWAQ, which are suited for 1-D to 3-D water quality modelling (see next paragraph). Then the ODE's are embedded in partial differential equations (PDE's) describing the change of a state variable in time and space. Also lattice differential equations (LDE's) can be used for spatial modelling, and are supported as well by DATM. PDE's have a continuous spatial structure, whereas LDE's are 
discrete in space. This discretization causes additional dynamics compared to PDE's (Chow et al. 1996). To avoid these effects of discretization, mostly PDE's are used in spatial water quality modelling.

To illustrate how a model - consisting of processes described by ODE's can be embedded in LDE's and PDE's, consider an ODE describing how the change of substance $C$ with time depends on an inflow (with concentration $\left.C_{i n}\right)$, an outflow and the model term $f_{R}(C, t)$ describing the model processes for substance $C$ :

$$
\frac{\mathrm{d} C}{\mathrm{~d} t}=a\left(C_{i n}-C\right)+f_{R}(C, t),
$$

where $a$ is the dilution rate, which is the inverse of the water retention time. A spatial dimension can be added by regarding spatially connected compartments, which can be described by LDE's. For a chain of compartments where compartment $i$ receives water from the upstream compartment $i-1$ this results in

$$
\frac{\mathrm{d} C_{i}}{\mathrm{~d} t}=a\left(C_{i-1}-C_{i}\right)+f_{R}\left(C_{i}, t\right)+d\left(C_{i-1}-2 C_{i}+C_{i+1}\right),
$$

where dispersion of substances in space - with dispersion rate $d$ - comes into play. Note that each compartment is a perfectly-mixed discrete spatial unit. For a continuous spatial structure, the change of substance $C$ with time at a certain location can be described by a PDE:

$$
\frac{\partial C}{\partial t}=-\nabla \cdot(\vec{u} C)+f_{R}(C, t)+D \nabla^{2} C,
$$

where the first term describes the transport of $C$ by flow (advection) related to flow velocity $\vec{u}=\left(u_{x}, u_{y}, u_{z}\right)$ and the third term describes the transport by dispersion, where $D$ is the dispersion coefficient.

In each case, DATM provides a framework independent description of the process terms $f_{R}(C, t)$ and merges these with the framework specific hydrodynamic terms in a format prescribed by the framework.

\section{Frameworks}

We used the frameworks ACSL, GRIND for MATLAB, OSIRIS and R (here all used for 0-D modelling), and DELWAQ and DUFLOW (used for 1-D to 3-D modelling). These frameworks were chosen for their capabilities but also for practical reasons, such as the availability of the framework and the experience of one or more of the authors with a given framework. We summarise technical details in Table 5.1, such as the programming language - which is 
Table 5.1. Technical details of the frameworks used for water quality modelling with PCLake and PCDitch.

\begin{tabular}{|c|c|c|c|c|c|}
\hline Framework & $\begin{array}{l}\text { Programming } \\
\text { language }\end{array}$ & $\begin{array}{l}\text { Language } \\
\text { type }^{\mathrm{a}}\end{array}$ & User-interface $^{\mathrm{b}}$ & $\begin{array}{l}\text { Automatic equation } \\
\text { sorting }\end{array}$ & Availability \\
\hline ACSL & ACSL & $\mathrm{c}$ & $\mathrm{cl} / \mathrm{GUI}$ & yes & paid license \\
\hline GRIND & MATLAB & $\mathrm{i}^{1}$ & $\mathrm{cl} / \mathrm{GUI}^{2}$ & yes & free $^{3}$ \\
\hline OSIRIS & C++ & $\mathrm{c}$ & $\mathrm{cl} / \mathrm{GUI}^{4}$ & no & free \\
\hline $\mathrm{R}$ & $\mathrm{R}$ & $\mathrm{i}^{1}$ & $\mathrm{cl} / \mathrm{GUI}$ & no & free \\
\hline DELWAQ & FORTRAN & $\mathrm{c}$ & $\mathrm{cl} / \mathrm{GUI}$ & no & free \\
\hline DUFLOW & DUPROL $^{5}$ & $\mathrm{c}$ & GUI & no & free \\
\hline
\end{tabular}

${ }^{\mathrm{a}} \mathrm{c}=$ compiled, $\mathrm{i}=$ interpreted, ${ }^{\mathrm{b}} \mathrm{cl}=$ command-line, GUI $=$ Graphical User Interface, ${ }^{\mathrm{c}}$ automatic sorting guarantees that variables are not used until they are assigned a value, ${ }^{1}$ models can be compiled in C++ (MATLAB) or also in FORTRAN (R) and linked to MATLAB or R to increase runtime performance, ${ }^{2}$ to enter the equations as Forrester diagrams (Forrester 1961), ${ }^{3}$ GRIND runs in MATLAB which is not free of charge, ${ }^{4}$ Microsoft Excel or Microsoft Access, ${ }^{5}$ DUFLOW Program Language.

important for the runtime performance -, the user-interface and the licensing policy.

\section{ACSL}

The Advanced Continuous Simulation Language (ACSL) is among the first modelling frameworks used to simulate continuous systems of time-dependent nonlinear differential equations (Mitchell and Gauthier 1976). It is an equationoriented language developed to represent mathematical models in an easily readable way. ACSL includes a MACRO capability to duplicate (sets of) states. In ecosystem modelling, this characteristic can be used to implement species within functional groups (for example see Janse 2005).

GRIND for MATLAB

GRIND for MATLAB (hereafter referred to as GRIND and based on the C program Great Integrator Differential equations (De Boer and Pagie 1983)) is a modelling framework used to analyse time-dependent differential equations and difference equations as well as matrix and vector models (http:/ /www.sparcscenter.org/grind). GRIND is developed for theoretical ecology and features phase-plane and bifurcation analyses. It is mainly used to analyse simple models with a few equations, and for teaching purposes.

\section{OSIRIS}

The Object-oriented Simulation Framework for Individual-based Simulations (OSIRIS) is a modelling framework that was originally developed for the imple- 
mentation of event-driven spatially-explicit individual-based models (Mooij and Boersma 1996). It was extended, however, to implement models of differential equations. A particular feature of OSIRIS is that the input and output files are structured in a database. DATM can be seen as an extension of this design.

$\mathrm{R}$

$\mathrm{R}$ is a programming language and environment developed for statistical computing and graphics (R Core Team 2013). It attracted the attention of the scientific community and has gained much popularity in recent years, as a result of its open licensing under the GNU General Public License and well documented package system (Fox 2009). This has promoted the communitybased development of more than five thousand add-on packages. One of these packages, deSolve, allows for a simple implementation of dynamic models based on differential equations (Soetaert et al. 2010).

DELWAQ

DELWAQ (Delft Water Quality) is a water quality module embedded in the hydrodynamic framework Delft-3D or SOBEK (Delft Hydraulics 1995, Deltares 2013). It is used for 1-D, 2-D and 3-D water quality modelling in seas, estuaries, streams, ditches and lakes. Given externally calculated hydrodynamics, DELWAQ simulates the transport of substances and sediment in a user-defined spatial configuration. Built-in water quality processes can be switched on or off by the user. The user can install additional processes, thus allowing to link DELWAQ with models such as PCLake.

\section{DUFLOW}

The DUFLOW (Dutch Flow model) water quality modelling framework was originally developed for and used by Dutch Water Boards for simulating 1-D unsteady flow in streams and ditches (Clemmens et al. 1993, Spaans et al. 1989). Later, it was extended to simulate water quality in 1-D. Water quality models can be implemented in Duflow as a set of differential equations. The process equations are evaluated for each hydrological unit when simulating the transport of substances in a user-defined spatial configuration. 


\section{Models}

PCDitch

PCDitch describes ditch ecosystems (Janse 1998). It covers six functional groups of macrophytes - essential for the ecological functioning of ditches (Portielje and Roijackers 1995) - and one group of algae (Fig. 5.1). These groups compete for nutrients and light, each with a different competition strategy as defined by their growth form. PCDitch describes the cycling of dry weight and nutrients ( $\mathrm{N}$ and $\mathrm{P}$ ) for all model compartments, in both the upper sediment layer and the water column. PCDitch is mainly used to study the critical nutrient loading at which ditches become dominated by free-floating plants instead of submerged plants (van Liere et al. 2007). This critical loading is relevant for management because dense mats of free-floating plants form a threat to biodiversity (Scheffer et al. 2003).

PCLake

PCLake describes shallow lake ecosystems (Janse 1997). It is a food web model that covers the interaction between different trophic levels (fish, zooplankton and primary producers) within an ecosystem context. Similarly to PCDitch, it includes the water column and the upper sediment layer and describes the cycling of dry weight and nutrients ( $\mathrm{N}$ and $\mathrm{P}$ ) over the different model components (Fig. 5.1). PCLake is primarily used to define critical nutrient loadings at which a vegetation-dominated clear-state turns into a phytoplankton-dominated turbid-state or vice versa. Critical transitions to and from a turbid state often occur at different nutrient loadings, implying alternative stable states. PCLake has proved successful in answering both fundamental and applied questions and therefore bridges the gap between these two (Mooij et al. 2010).

\subsection{Results}

\section{Runtime performance}

Before analysing the models in the different frameworks, we first determined the runtime of the models per framework-implementation. Not surprisingly, the runtime differed considerably between frameworks (Table 5.2). The runtime of a 50-year PCDitch and PCLake run (default settings) was of the order of seconds using OSIRIS and ACSL, of the order of minutes using DUFLOW and DELWAQ and of the order of hours using GRIND and R. The latter frameworks 

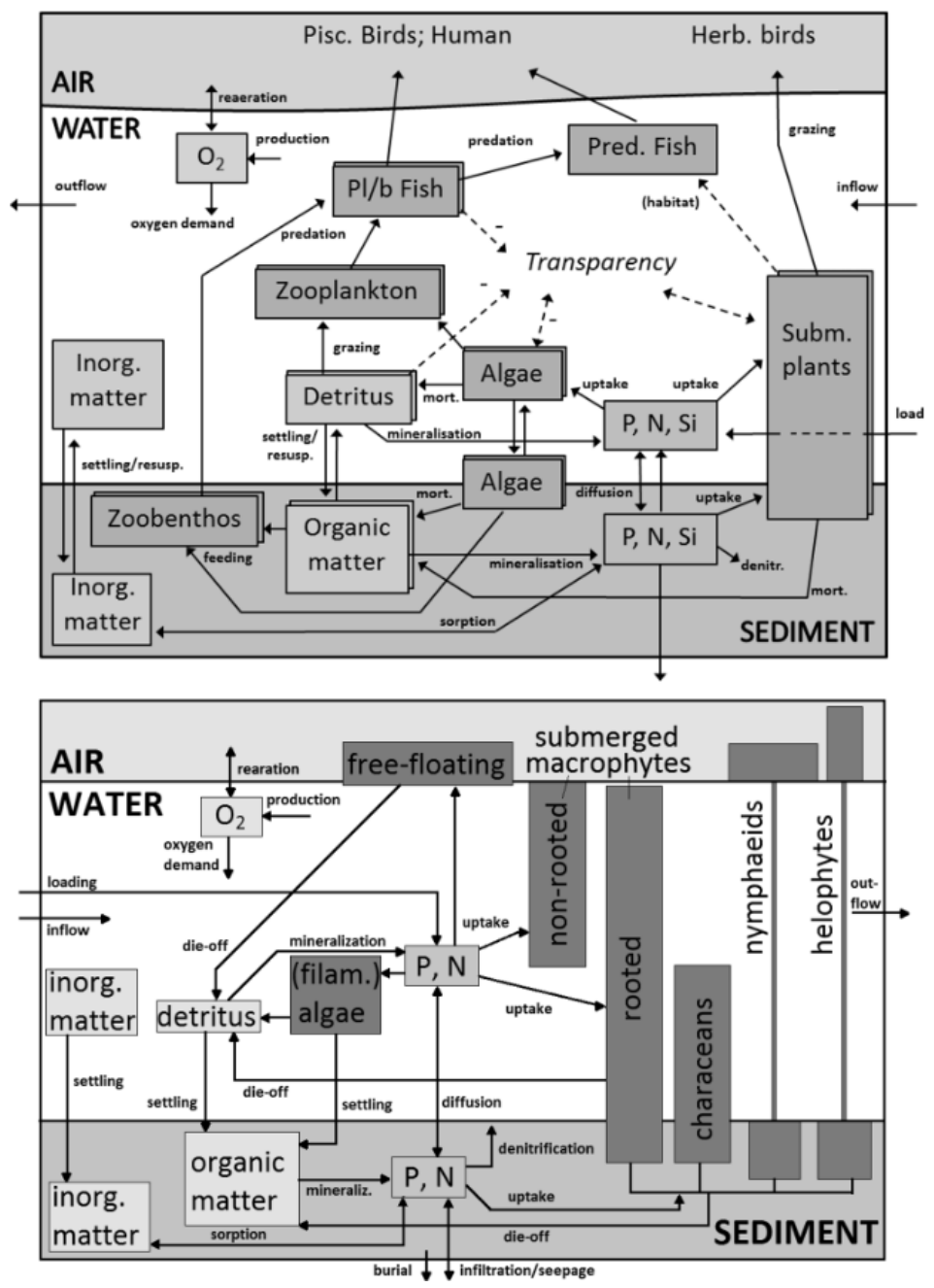

Figure 5.1. Model structure of PCLake (upper figure) and PCDitch (lower figure), modified from Janse (1997) and Janse (1998).

use interpreted languages, which explains the long runtimes. However, in both GRIND and R, the models could be compiled using $\mathrm{C}++$ and then called from the framework. This reduced the runtime considerably - up to 5000 times - leading to runtimes similar to OSIRIS and ACSL. The longer runtimes of the spatially explicit frameworks DUFLOW and DELWAQ were, expectedly, due to the fact that they solved PDE's instead of ODE's and used integrators 
with a fixed time step, whereas the other frameworks used integrators with a variable time step (see Table A.1 in Appendix A).

Table 5.2. Runtime (min:sec) of a 50 -year run of PCDitch and PCLake in different frameworks while producing daily output for all state variables. We used the preferred integrators (Table A.1) with default settings on absolute and relative tolerance. The integrators had a variable time step, except those of DELWAQ and DUFLOW whose fixed time step was set to 10 minutes. The calculations were performed on a standard desktop PC with Intel Core i5-2500 @ 3.30 GHz processor.

\begin{tabular}{lcccccc}
\hline & ACSL & GRIND & OSIRIS & R & DELWAQ & DUFLOW \\
\hline PCLake & $0: 03$ & $0: 02^{1} / 30: 32$ & $0: 06$ & $0: 03^{1} / 284: 25$ & $9: 04^{2} / 10: 29^{3}$ & $16: 16$ \\
PCDitch & $0: 19$ & $0: 16^{1} / 186: 36$ & $0: 37$ & $0: 02^{1} / 179: 25$ & $7: 37^{2}$ & $6: 23$ \\
\hline \multicolumn{7}{l}{ model code was compiled in C++ and then called from the framework } \\
2 model was run in 1-D environment with hydrodynamics calculated by SOBEK \\
3 model was run in 3-D environment with hydrodynamics calculated by Delft-3D
\end{tabular}

\section{Analysis}

The multi-framework implementation of PCLake and PCDitch allowed us to have a great amount of choice and versatility in the methodologies for analysis, fully validating the purpose of DATM. We carried out sensitivity analyses, calibration/ validation/ uncertainty analyses and bifurcation analyses using built-in tools (GRIND and OSIRIS) and by writing our own scripts (ACSL and R). Furthermore, we used DELWAQ and DUFLOW for spatial scenario analyses. Table 5.3 gives an overview of the published and unpublished analyses on PCLake and PCDitch that we are aware of, classified per analysis and framework. Note that the analyses that date back to before 2012 were not carried out with DATM-implementations of the models, but resulted from non-automated framework-to-framework translation of the models. We report on these pre-DATM analyses, as they illustrate the tools provided by the different frameworks for model analysis. They also illustrate the need to run a model in multiple frameworks to address multidisciplinary questions. This aspect, together with the realization of how much effort it took to translate a model from one framework to another, led, in fact, to the development of DATM. In addition to reporting on the published and unpublished analyses (Table 5.3) - where the figures show only analyses resulting from DATM-implementations - we point to powerful framework tools that come within reach through using DATM. 
Table 5.3. Overview of the published and unpublished analyses on PCDitch (in bold) and PCLake, classified per analysis and framework. $\mathrm{X}=$ already performed, where studies that used the DATM-approach are underlined, $\mathrm{x}=$ potentially to be performed.

\begin{tabular}{|c|c|c|c|c|c|c|}
\hline Analysis & ACSL & GRIND & OSIRIS & $\mathrm{R}$ & DELWAQ & DUFLOW \\
\hline Sensitivity analysis & $\mathrm{X}^{1}$ & $\mathrm{X}^{2}$ & $\mathrm{X}^{\underline{3}}$ & $\mathrm{x}$ & & \\
\hline Calibration & $X^{1,4,5}$ & $\mathrm{X}^{\underline{6}}$ & $\mathrm{X}^{3}, \underline{7}$ & $\mathrm{x}$ & & \\
\hline Validation & $\mathrm{X}^{8}$ & $\mathrm{x}$ & $\mathrm{X}^{\mathrm{7}}$ & $\mathrm{x}$ & & \\
\hline Uncertainty analysis & $\mathrm{X}^{1,5}$ & $\mathrm{x}$ & $\mathrm{X}^{3}, \underline{9}$ & $\mathrm{x}$ & & \\
\hline Bifurcation analysis & $X^{10-13,14}$ & $\mathrm{x}$ & $X \underline{9}$ & $\mathrm{X} \underline{15}$ & & \\
\hline \multicolumn{7}{|l|}{ Scenario analysis } \\
\hline $0-\mathrm{D}$ & $X^{13,14,16-21,22,23}$ & $\mathrm{x}$ & $X^{24-27}$ & $X^{\underline{28}}$ & & \\
\hline 1-D to 3-D & & & & & $x^{29}$ & $X^{30, \underline{31}}$ \\
\hline \multicolumn{7}{|c|}{$\begin{array}{l}{ }^{1} \text { Janse et al. (2010), }{ }^{2} \text { see Fig. } 5.2,{ }^{3} \text { Nielsen et al. (2014), }{ }^{4} \text { Janse (1998), }{ }^{5} \text { Aldenberg et al. (1995), }{ }^{6} \text { Elzinga } \\
\left.(2013),{ }^{7} \text { Trolle et al. (2014) (see Fig. 5.3), }{ }^{8} \text { van Puijenbroek et al. (2004), }{ }^{9} \text { Lischke et al. (2014) (see Fig. } 5.4\right) \\
{ }^{10} \text { Janse et al. (2008), }{ }^{11} \text { Mooij et al. (2009), }{ }^{12} \text { Janse (1997), }{ }^{13} \text { Janse et al. (1998), }{ }^{14} \text { van Liere et al. (2007), }{ }^{15} \\
\text { unpublished results, }{ }^{16} \text { Mooij et al. (2007), }, 17 \text { Janse et al. (2001), }{ }^{18} \text { Witteveen + Bos (2008a), }{ }^{19} \text { Witteveen } \\
+ \text { Bos (2008b), }{ }^{20} \text { Witteveen + Bos (2009), }{ }^{21} \text { Witteveen + Bos (2010a), }{ }^{22} \text { Witteveen + Bos (2010b), }{ }^{23} \text { Janse } \\
\text { and van Puijenbroek (1998), }{ }^{24} \text { Sollie et al. (2008), }{ }^{25} \text { Witteveen + Bos (2013a), }{ }^{26} \text { Witteveen + Bos (2013b), }{ }^{27} \\
\text { Witteveen + Bos (2013c) },{ }^{28} \text { Broers (2012), }{ }^{29} \text { see Fig. 5.6, }{ }^{30} \text { van Liere et al. (2002), }{ }^{31} \text { see Fig. 5.5. }\end{array}$} \\
\hline
\end{tabular}

\section{Sensitivity analysis}

A sensitivity analysis quantifies how changes in the model input (i.e., parameters, initial states or external inputs) affect the model outcome (Klepper 1997). We used sensitivity analyses to identify the most sensitive parameters. This gives insight into model behaviour and can be used to select parameters for calibration.

With PCLake, ACSL with SIMLAB (EC-JRC-ISIS 2002) was used to carry out a stepwise sensitivity analysis (Janse et al. 2010) by (1) screening the parameters to select a subset of most sensitive ones using the Morris method (Morris 1991) and (2) performing a global sensitivity analysis on this subset using the FAST ('Fourier Amplitude Sensitivity Test') method (Saltelli et al. 2008). With PCDitch, we used GRIND to calculate sensitivity indices for the parameters by Monte Carlo sampling followed by a regression. This information was used to cluster parameters with a similar or opposite effect on the model outcome (Fig. 5.2), showing that the parameters of a certain functional group of water plants in PCDitch are closely linked. ACSL, GRIND and OSIRIS offer basic One-At-a-Time (OAT) sensitivity analysis tools (Saltelli et al. 2008). These tools were not used, because they are less suited for non-linear models like PCDitch and PCLake than the tools described above. 

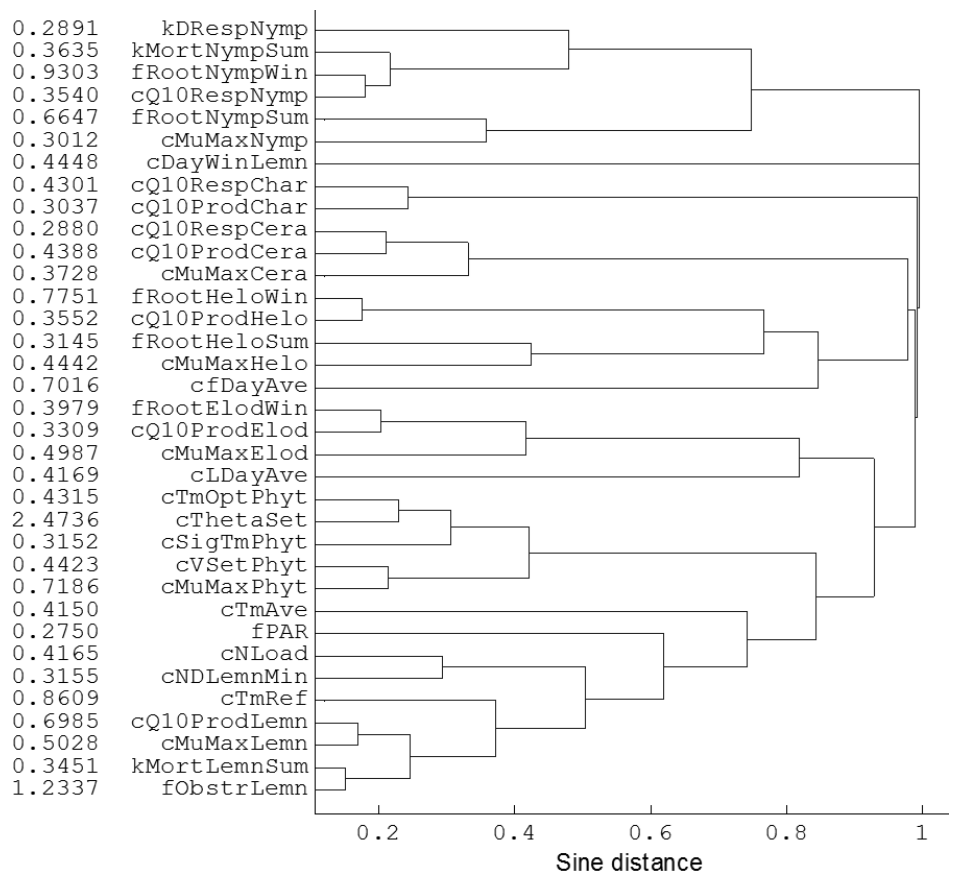

Figure 5.2. Example of a sensitivity analysis for PCDitch performed with GRIND (based on Klepper 1989). This dendrogram shows clusters of the most sensitive model parameters with a similar or opposite effect on the model results (biomass of all water plants at several times during the run). The 'sine distance' is used as a similarity measure of the parameters. The value of the sensitivity index before each parameter is the length of the vector of the sensitivity coefficients which is a measure of the total strength of the effect of the parameter. For more details see Klepper (1989) and van Nes et al. (2002).

R offers a variety of sensitivity tools. For example the Flexible Modelling Environment (FME) package (Soetaert and Petzoldt 2010) contains functions for global and local sensitivity analyses. FME can also evaluate the identifiability of parameter sets, which is useful for over-parameterized models like PCLake and PCDitch (Mieleitner and Reichert 2006). Non-identifiability occurs commonly in PCLake and PCDitch, as a change in one parameter can often be compensated by changing other parameters (Janse et al. 2010).

\section{Calibration}

Calibration aims at improving the fit between a model and measured data. Various optimization techniques exist that randomly or actively search parameter space for the best fit. The fit is usually measured through the root 
mean squared error (RMSE) or mean relative error (MRE) (e.g. Trolle et al. 2014). Calibration can also be regarded as a way to find reasonable values for poorly defined or unmeasurable parameters (inverse modelling), a situation that is common for most environmental models (van Oevelen et al. 2010).

Using ACSL with SIMLAB, PCDitch was calibrated against experimental ditches by Simulated Annealing (van Laarhoven and Aarts 1987) and PCLake was calibrated - using a Bayesian procedure - on 43 mainly Dutch shallow lakes, aiming at a compromise fit rather than calibration on a specific lake (Janse et al. 2010), where the state of the lake (turbid or clear) was predicted well for $91 \%$ of the lakes. The outcome of the multi-lake calibration can be used as a starting point for the optimization of parameters for a specific lake. For example, the OSIRIS implementation was used to calibrate PCLake against two Danish shallow lakes: Lake Arreskov (Nielsen et al. 2014) and Lake Engelsholm (Fig. 5.3) for which a reasonable fit was obtained between measured and simulated algae biomass from 1999 until 2001. In the latter study an ensemble of parameters combinations and ranges was used that would allow the simulated output time series to encompass all or most of the observations. In another study, PCLake was calibrated against the Dutch large shallow lake Markermeer (Elzinga 2013) by using GRIND for local optimization (simplex method: Press et al. 2009) and global optimization (shuffled complex evolution (SCE-UA) method).

R also offers a variety of calibration tools (see http://cran.r-project.org/web/ views/Optimization.html).

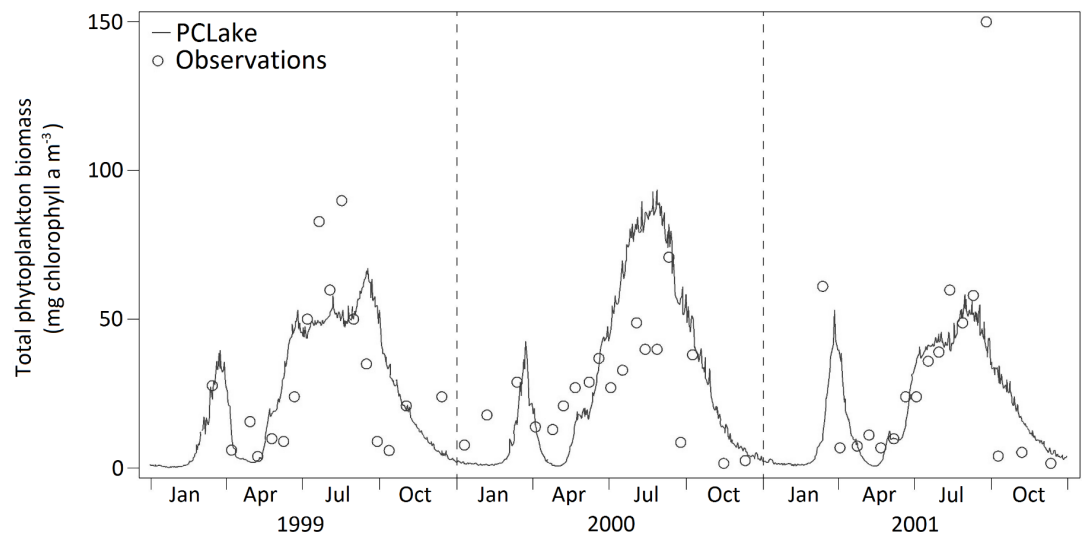

Figure 5.3. Example of calibration (years 1999-2000) and validation (year 2001) of PCLake performed with OSIRIS for Lake Engelsholm, a shallow eutrophic lake in Denmark (figure was modified from Trolle et al. 2014). 


\section{Validation}

There are many different definitions for model validation. Here, we refer to it as 'testing whether a model is acceptable for its intended use' (Refsgaard and Henriksen 2004). This is often done by confronting a model with measured data of systems that were not used for model calibration. In general, validation involves computing the goodness of fit between simulated and measured data and analysing whether the residuals are random or systematic.

Using ACSL, PCLake was validated against data for 34 Dutch shallow lakes (van Puijenbroek et al. 2004) and on 9 lakes that were not used for the Bayesian calibration procedure referred to above (Janse et al. 2010), using MRE as the fit criterion.

GRIND and OSIRIS provide basic $\mathrm{R}^{2}$ validation tools and $\mathrm{R}$ offers more specific validation tools, such as in the packages FME and qualV (Jachner et al. 2007).

\section{Uncertainty analysis}

Uncertainty analysis measures the reliability of model output given uncertainties in model input, initial values and model structure (O'Neill and Gardner 1979).

The Bayesian procedure already applied for calibration (Janse et al. 2010) served to quantify uncertainties in the critical nutrient loading computed by PCLake in ACSL. Uncertainty ranges were computed from posterior parameter distributions; i.e. prior parameter distributions that were narrowed down by validating modelled output against observation data. The OSIRIS implementation was used to evaluate uncertainty of PCLake output, based on 900 simulations with randomly sampled values from a uniform distribution for the most sensitive parameters, sampled within a range of $-20 \%$ to $+20 \%$ of their default value (Nielsen et al. 2014). The same OSIRIS implementation was used for a simple structural uncertainty analysis, as OSIRIS can be directed from a database environment which allows for comparing different versions of the model code, facilitating structural uncertainty analysis. OSIRIS was used to change the structure of PCLake by adding organic matter in three different ways (Lischke et al. 2014). This addition affected the hysteresis curve - which indicates at which nutrient loadings the lake switches from a clear to turbid state and vice versa - such that organic matter input increases the chance for a lake to become or stay turbid (Fig. 5.4). GRIND and R also include tools for uncertainty analysis. In GRIND the Monte Carlo sampling method used for sensitivity analysis can also be used for a 
classical uncertainty analysis where the effects of prior distributions of parameters on the model outcomes can be evaluated (van Nes and Scheffer 2003). R provides a variety of tools related to model input uncertainty (see http://cran.r-project.org/web/views/Bayesian.html) and some packages facilitate structural uncertainty analysis (for example simecol (Petzoldt and Rinke 2007)).

\section{Bifurcation analysis}

Bifurcation analysis is used to reveal qualitative changes in long-term (asymptotic) model behaviour due to changes in parameters (e.g., mortality rates) or external forcings (e.g., nutrient loading). It can be used to determine the shape of the ecological stability landscape (Scheffer et al. 2001). The potential of this technique to analyse complex simulation models is easily overlooked.

We carried out bifurcation analyses in $\mathrm{R}$ to find critical nutrient loadings for PCLake (not shown here), leading to a hysteresis curve like in Fig. 5.4.

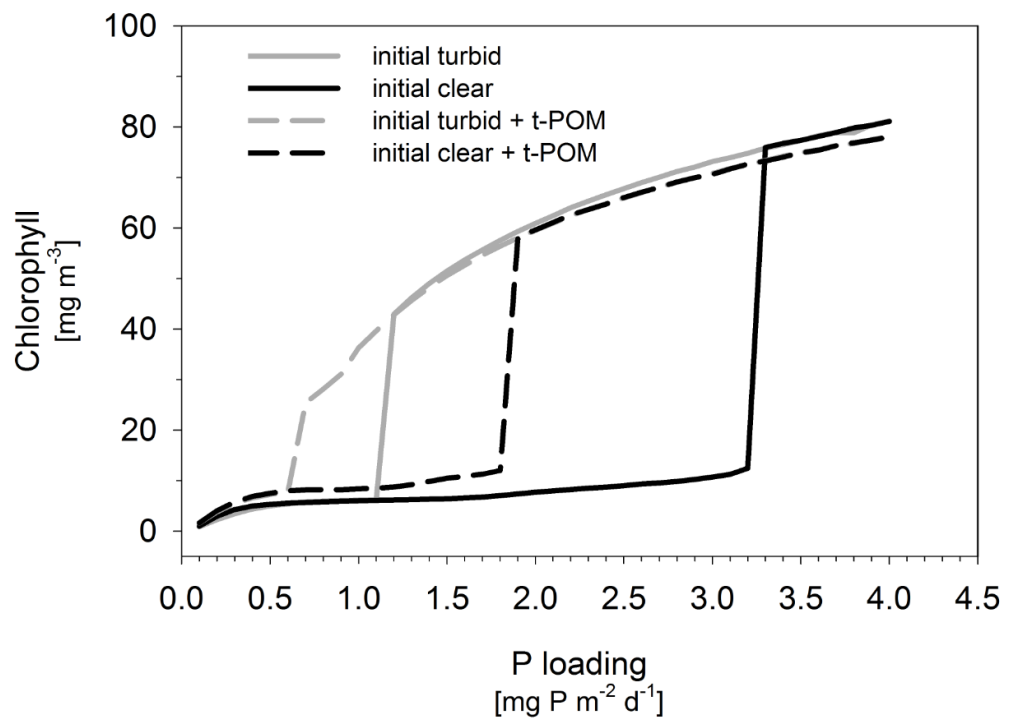

Figure 5.4. Example of a bifurcation analysis combined with a structural uncertainty analysis of PCLake in OSIRIS (figure copied from Lischke et al. 2014). It shows the bifurcation points of an average temperate shallow lake - the critical external phosphorus loadings at which the lake switches from a clear state to a turbid state and vice versa - for different model structures, so whether allochtonous terrestrial particulate organic matter (t-POM) is taken into account or not. The turbidity is represented by the average chorophyll-a concentration in the last year of a 30 year run and the t-POM input (only in autumn) equals $8 \mathrm{~g} \mathrm{DW} \mathrm{m}^{-2}$ day $^{-1}$ to mimic leaf fall. 
For PCDitch, ACSL was used for this purpose (van Liere et al. 2007), where no hysteresis was found because the critical nutrient loading towards and from duckweed dominance was the same. These bifurcation analyses can be combined with scenario evaluation. For example, Janse et al. (2008) studied the importance of basic system characteristics (e.g. depth, fetch, sediment type) in PCLake using ACSL. Others focused on the effects of global warming (Mooij et al. 2009, Mooij et al. 2007) using OSIRIS.

OSIRIS provides a simple bifurcation tool that calculates the effect of a stepwise varied parameter on a response variable. This tool is extensively used in teaching. We wrote scripts with a similar procedure for ACSL and R. GRIND features the automated 'paranal' routine for this approach. For more powerful bifurcation analyses, a switch to specialized frameworks, such as AUTO (Doedel and Oldeman 2009) or MATCONT (Dhooge et al. 2003), would be preferred. These frameworks are mainly used to analyse minimal dynamic models, consisting of only a few equations models (e.g. Kooi 2003), but could also be used for more complex models.

\section{Scenario analysis}

With a calibrated and validated model, scenario analyses can be carried out to evaluate potential future scenarios and the effectiveness of measures for ecosystem management.

The effect of nutrient loading on transient dynamics of floating and submerged plants were explored with PCDitch in ACSL (Janse and van Puijenbroek 1998). Using the same implementation, the effect of sediment type,

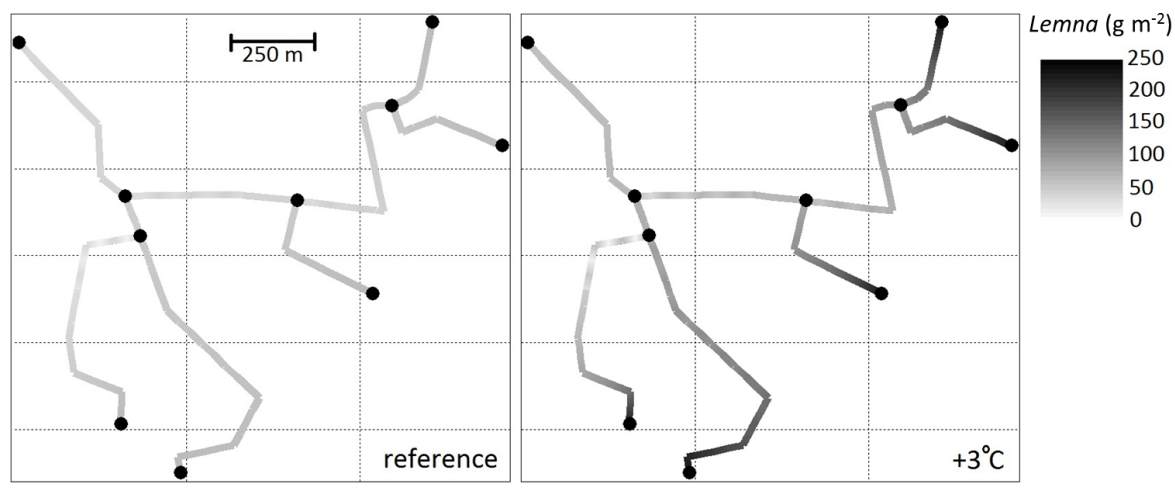

Figure 5.5. Example of a 1-D scenario analysis for PCDitch performed with DUFLOW. It shows the duckweed biomass on a summer day in a spatial network of ditches for a reference year (left) and a $3^{\circ} \mathrm{C}$ warmer year (right). 
flow rate and water depth on the critical nutrient loading was studied (van Liere et al. 2007). With PCLake in ACSL, the impact on the critical nutrient loading of herbivory by birds and fish (Janse et al. 1998), of global warming (Mooij et al. 2007) and of the size of surrounding marsh zone (Janse et al. 2001). The nutrient removal capacity of the marsh zone, leading to lower in-lake nutrient concentrations, was assessed with PCLake in OSIRIS (Sollie et al. 2008). We performed 1-D scenario tests with PCDitch in DUFLOW by looking at the effect of global warming on duckweed abundance in a spatial network of ditches (Fig. 5.5), showing that duckweed benefits from higher temperatures at the cost of submerged water plants. With PCLake in DELWAQ we performed a 3-D scenario analysis in the large shallow Chinese lake Taihu (Fig. 5.6) showing that the occurrence of summer algal blooms depends on the history of the lake, such as whether the lake was initially clear or turbid.

GRIND and R (using package deSolve) offer the possibility to define events, by making sudden changes in the values of state variables. This can be used to mimic discrete events in ecosystem management such as the removal of fish.
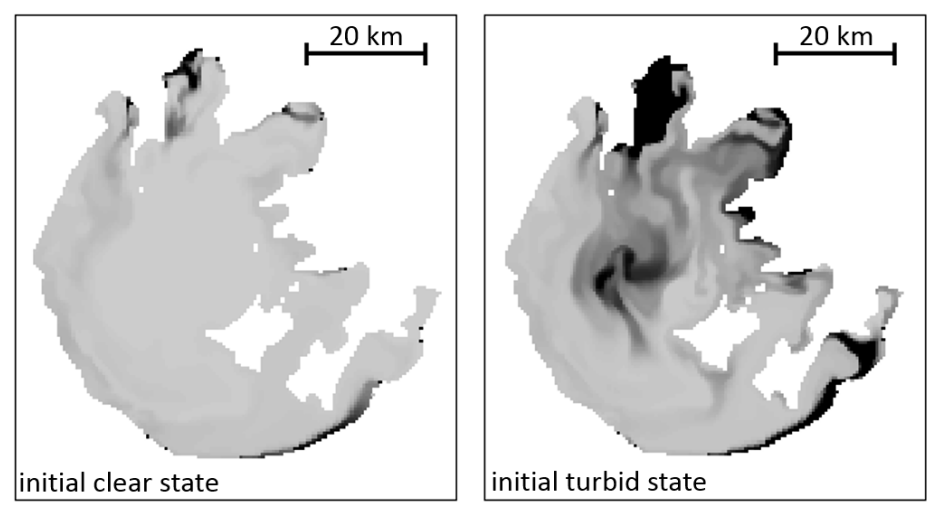

Cyanobacteria $\left(\mathrm{g} \mathrm{m}^{-3}\right)$

Figure 5.6. Example of a 3-D scenario analysis with PCLake in DELWAQ. It shows preliminary results of summer algal blooms in the large shallow Chinese lake Taihu, when starting from a clear water state (left) and a turbid state (right).

\subsection{Discussion}

Water quality modelling deals with multidisciplinary questions ranging from fundamental to applied. This diversity in questions requires a multitude of analysis techniques and therefore a multitude of frameworks to run a model in. 
Here we applied a database approach to modelling (DATM) for this purpose, which facilitates the implementation of a model in a framework and makes it easy to switch between frameworks to make use of the myriad of analysis tools offered by the frameworks. We would like to stress that we advocate the idea behind DATM rather than the specific implementation that we created. This idea is to put the process formulations of water quality models (terms $f_{R}(C, t)$ in Eq. 5.1-5.3) in a framework independent database and write translators to merge these process formulations with framework specific features such as spatial discretization and hydrodynamical process formulations. We are aware that many modelling packages contain a framework dependent library of process routines (e.g. DELWAQ, DUFLOW, etc.). From here it is only a small step to DATM. We see the technical simplicity of DATM as a strength and would welcome alternative implementations of the idea.

In this study we implemented PCDitch and PCLake in six different frameworks which revealed two clear benefits of DATM: 1) the possibility to use the framework one is familiar with for many analyses, and 2) the possibility to switch easily to other frameworks to exploit additional tools. This includes the switch between a 0 -D and 1-D to 3-D implementation of the model. The first benefit arises from the redundancy in analysis tools amongst frameworks, while the second benefit stems from their complementarity. A surprising side effect of our efforts was that it ignited a healthy competition among the developers of some of the frameworks (in particular, GRIND and OSIRIS) to include missing tools in their framework after being convinced of their usefulness in other frameworks. The field of water quality modelling can undoubtedly benefit from such cross-fertilization of ideas.

The comparison of model runtimes in the different frameworks revealed huge differences. It showed the amount by which a model runs faster - up to 5000 times - in a framework that uses a compiled programming language instead of an interpreted language. This gain in runtime will especially pay off for large models when performing multiple model runs for example for a sensitivity or bifurcation analysis. Fortunately, some frameworks like R and GRIND which are based on interpreted languages offer the possibility to compile the model code to increase runtime performance. Furthermore, there is an obvious computational cost to implement a model in a spatial setting, increasing the runtime up to 100 times or more (dependent on the spatial configuration) compared to a 0 -D setting. When applying models spatially, it is therefore recommendable to first explore the model behaviour in a 0 -D setting. 
Increasing redundancy among frameworks may lead to a point where one can ask what the added value is of using an approach like DATM over using encompassing frameworks such as the Delta Shell (Donchyts and Jagers 2010) or FABM (Trolle et al. 2012), which are both currently under construction. Indeed, an implicit motivation of many framework developers seems to be to make other frameworks superfluous. We note, however, that many of the frameworks from the early days of ecological simulation in the 1970s are still maintained even though many new frameworks have become available since then (Argent 2004). Based on this observation, we can reasonably expect that fragmentation of the field of water quality modelling, when it comes to framework use, is there to stay and can only be overcome by a DATM-like approach that operates at a different level.

While implementing PCDitch and PCLake in the six frameworks and discussing our results with experts in the field, we were pointed to a number of other frameworks for which DATM translators will be useful, either because of their additional tools, or simply because they are extensively used. Among these frameworks are AUTO (Doedel and Oldeman 2009), FABM (Trolle et al. 2012), FST (van Kraalingen et al. 2003), MATCONT (Dhooge et al. 2003), Mathematica (Wolfram 1999), Python (van Rossum and Drake 2001), Simecol (Petzoldt and Rinke 2007), SIMILE (Muetzelfeldt and Massheder 2003), SMART (Grant and Lai 1998) and Stella (Richmond and Peterson 1985).

In addition to the findings of this study, a database approach like DATM may facilitate the inspection and review of the assumptions underlying model code (Mooij et al. 2014). It offers a transparent way to inspect and review a model through standard database queries that can select the groups of states, parameters or equations to be studied in detail. This is especially rewarding for large models like PCDitch and PCLake, as demonstrated by our collaborative projects and student courses. Framework review, which, like model review, is often lacking in the scientific review process, can also be greatly enhanced with a database approach, by checking that a model gives the same results when implemented in another framework (Joppa et al. 2013). Furthermore, an approach like DATM provides a framework-independent way to store a given model version and a common ground for multiple users to work on a given version, even if these users prefer different frameworks for model analysis. This helps to maintain coherence in model development and promotes community-based model development.

In this study, we reported on our findings with applying DATM in the field of water quality modelling with fairly complex models. Our results are 
directly applicable, however, to other process models that are based on ODE's, whether simple or complex. These models can then be implemented by DATM in a 0-D setting or a spatial setting (PDE's). There are nonetheless some limitations. For instance, physiologically structured models of animal populations - that are defined in terms of PDE's with age, size, energy reserves and/or ontogenetic development as one of the integration variables (de Roos and Persson 2012) - cannot yet be implemented in the current definition of DATM, just like discrete time models, and the structured versions thereof, such as population matrix models (Caswell 1989). We see it as a future challenge to implement these types of models in DATM. The potential of DATM to easily combine models and frameworks does not imply that tools that were developed for the analysis of the simpler models will always work for more complex models. For instance, the sophisticated bifurcation tools that continue (un)stable equilibria along one or two parameter axes do not apply to seasonally forced models like PCDitch and PCLake, but only work for models with a constant forcing leading to stable steady states or periodic solutions like limit cycles.

We conclude that a database approach to modelling can be useful to address multidisciplinary questions in water quality modelling, as it makes the multitude of analysis techniques provided by different frameworks easily accessible. Thereby, it allows one to fully exploit the strength of each framework. We envision that a community-based further development of the concept can contribute to the future development of water quality modelling by facilitating the exchange of models and process formulations within the community of water quality modellers.

\section{Acknowledgements}

We thank Peter Leffelaar, Huub Scholten, Anne van Dam and Martin Droog for their helpful discussions and comments during the development of this paper. This work is financed by the Netherlands Foundation for Applied Water Research (STOWA) project no. 443237 and the China-Netherlands Joint Scientific Thematic Research Programme (JSTP) of the Netherlands Organisation for Scientific Research (NWO) project no. 842.00.009. This is manuscript 5750 of the NIOO-KNAW. 


\section{Appendix A: Implementing PCDitch and PCLake in the frameworks by using DATM}

\section{Step 1: Building the DATM translator}

Framework-specific DATM translators turn essential model information stored in the database - into an operational implementation of the model in the framework of choice. Essential model information includes the model equations and the information needed to run the model such as initial values for the state variables, parameter values, boundary conditions and runtime options (Mooij et al. 2014). We used Microsoft Excel to store, view and edit DATM information on PCDitch and PCLake and wrote the translators in Visual Basic for Applications (VBA).

The translators to GRIND and R were easiest to develop, typically taking a day, as they only dealt with translating the essential model information into the right syntax of the framework language. These two translators produced 'clean' and readable code with no overhead such as declaration statements and integration calls. These extra statements were needed by compiled language frameworks ACSL, OSIRIS, DUFLOW and DELWAQ. Translators for the spatially explicit frameworks DUFLOW and DELWAQ were most timeconsuming to write, typically taking a week. First, those parts of the model that handle the built-in flow of water and substances (see first term in Eq. 5.1) needed to be excluded, as flow is managed by the frameworks themselves. Thereafter, the model had to be linked to the hydrodynamic variables covered by the framework. Finally, model state variables that are subjected to flow (e.g. free-floating plants) had to be declared as such in the frameworks. The translators gathered this information from extra fields in the model database.

While developing the translators we experienced difficulties in translating 'dynamic parameters', i.e. parameters that are modified by the model as the simulation proceeds. For example, PCDitch and PCLake store year-to-year variations in phenological parameters that indicate the start of the growing season as such dynamic parameters. For the frameworks ACSL, DUFLOW, OSIRIS and R storing dynamic information as parameters is no problem. In GRIND and DELWAQ, however, we had to use framework-specific constructs to implement dynamic parameters.

\section{Step 2: Debugging the generated code and checking it at $t=0$}

All frameworks easily picked up syntax errors with the debuggers incorporated in their compilers or interpreters. This held particularly for integrated 
development environments, such as the free $\mathrm{C}++$ environment Code::Blocks (http://www.codeblocks.org/) which we used for compiling the OSIRIS code.

After syntax errors were resolved, we proceeded with checking for initial errors, i.e errors in the calculation of all identifiers in the model (parameters, initial states, auxiliary variables and derivatives) at $\mathrm{t}=0$, before numerical integration has started. The initial errors that we encountered included missing pairs of parentheses that resulted in an incorrect evaluation of the equations. Debuggers cannot detect such errors in equation logic. We therefore checked the calculated values against known correct output, in our case of the ACSL implementation of the models. Checking for initial errors proved to be a powerful tool. Indeed, at $\mathrm{t}=0$, errors in model equations are not yet propagated and the variables for which values do not match are direct clues to erroneous equations.

\section{Step 3: Choosing the integrator and setting up the simulation}

To run the model, the ordinary differential equations (ODE's) of PCLake and PCDitch had to be solved by numerical integration. The choice for an integrator and its step-size are important, as it influences the accuracy of results and model runtime performance. The various frameworks offer a list of different integrators to choose from (Table A.1). Highlighted in Table A.1 are the integrators that showed the best performances running PCDitch and PCLake in terms of accuracy and runtime in ACSL, GRIND, OSIRIS and R. In general, implicit integrators were more suited than explicit integrators, as PCLake and PCDitch contain stiff equations. Integrators that use a variable time step performed better in terms of runtime (up to an order of magnitude) than the ones that use a fixed time step while maintaining a good accuracy.

Model equations are solved differently in the spatially explicit frameworks (DUFLOW and DELWAQ). Here, the ODE's of PCLake and PCDitch are embedded as an extra term in the advection-dispersion equations (see the second term in Eq. 5.3). These are partial differential equations (PDE's) in time and space that describe the transport of substances, given previously calculated hydrodynamics (flow velocities). To solve these PDE's, DELWAQ has fourteen numerical integration methods to choose from, all with a fixed time step but varying implicitness. DUFLOW has one method of which the fixed time step and the implicitness of the time-integration can be set. For PCDitch, problems with negative state values were avoided by selecting a more implicit time-integration and a smaller time step, at the cost of a longer runtime. Note that in both DUFLOW and DELWAQ, the embedded PCLake or PCDitch term 
in the advection-dispersion equations (term 2 in Eq. 5.3) is always evaluated explicitly.

To set up the simulation, some frameworks required additional information next to the framework-specific model code and integrator. DUFLOW and DELWAQ required information on spatial configuration and associated boundary conditions to be defined in the user-interface. Furthermore, all frameworks required forcing functions to represent variable input (e.g. that of temperature and nutrient loading). For some frameworks (DUFLOW and DELWAQ), simulation options such as runtime and integration options, could only be defined in the user-interface and not passed to the framework by the DATM translators.

Table A.1. Available solvers per framework. The solvers of ACSL, OSIRIS, R and GRIND apply to ODE's, whereas the solvers of DELWAQ and DUFLOW deal with PDE's. In bold are the solvers that we preferred based on their performance in terms of numerical error and runtime.

\begin{tabular}{|c|c|}
\hline Framework & Solvers \\
\hline $\mathrm{ACSL}^{1}$ & Euler, rk2, rk4, rk2f, rk5f, Adams-Moulton, bdf (Gear's method) \\
\hline GRIND & Euler $^{2}$, rk $^{2}$, ode $5^{2}$, ode 23, ode 113 , ode 15 s, ode23s, ode $23 t$, ode23tb \\
\hline OSIRIS & Euler, rk4, rk45ck \\
\hline $\mathrm{R}^{3}$ & $\begin{array}{l}\text { Euler, rk2, rk4, rk23, rk23bs, rk34f, rk45f, rk45ck, rk45e, rk45dp6, rk45dp7, } \\
\text { rk78dp, rk78f, ode23, ode } 45 \text {, lsoda, lsode, lsodes, lsodar, vode, daspk, } \\
\text { radau, bdf, bdf_d, Adams, impAdams, impAdams_d, iteration }\end{array}$ \\
\hline DELWAQ $^{4}$ & $\begin{array}{l}\text { upwind scheme (US), second order Runge-Kutta, Lax Wendroff method, } \\
\text { alternating direction implicit method, flux-corrected transport scheme (FCTS) } \\
\text { implicit US with direct solver, implicit US with iterative solver, horizontal: US } \\
\text { and vertical: implicit in time and central discretisation (ITCD), horizontal: }\end{array}$ \\
\hline & $\begin{array}{l}\text { FCTS and vertical: ITCD, horizontal: US and vertical: implicit in time and } \\
\text { upwind discretisation (ITUD), horizontal FCTS and vertical: ITUD, horizontal: } \\
\text { implicit US and vertically: centrally discretised with iterative solver, ADI } \\
\text { scheme for 3D models with central discretization in the vertical, ADI scheme } \\
\text { for 3D models with upwind discretization in the vert., local-theta FCTS }\end{array}$ \\
\hline DUFLOW $^{5}$ & implicit scheme with direct solver \\
\hline
\end{tabular}

${ }^{1}$ Mitchell and Gauthier Associates (1995), ${ }^{2}$ Also available when the model is compiled using C++, ${ }^{3}$ Using R package 'deSolve' (Soetaert et al. 2010), ${ }^{4}$ Deltares (2013), ${ }^{5}$ Stowa and MX.Systems (2004)

\section{Step 4: Dynamic test runs}

As is common in dynamic test runs, we experienced runtime errors varying from small deviations from known correct output, to negative values of state variables, or even an early termination of the model run due to a division by zero. However, a proper check against initial errors (see step 2) usually 
prevented most runtime errors. Model implementation in hydrodynamic frameworks (e.g. DELWAQ) produced an extra type of runtime error related to incorrect communication between model and framework on hydrodynamics and boundary conditions. We identified and corrected these errors by comparing the water and nutrient balance for a single-cell model implementation in DELWAQ with a 0-D control model. In our case this was the OSIRIS-implementation of the models.

Once runtime errors were solved, dynamic test runs allowed testing framework performance, in terms of accuracy and runtime. Besides the inevitable small deviations of numerical origin, all frameworks that we tested produced the same output. 



\section{Chapter 6}

\section{General Discussion}

\subsection{New insights and implications for management}

\section{Alternative stable states in ditches}

The framework of alternative stable states is widely used in ecology to understand the response of ecosystems to slowly changing environmental stressors (Scheffer et al. 2001). This framework is well-developed and validated for shallow lakes to explain their clear versus turbid state and their resistance to switch to one of these contrasting states (Scheffer 1998). For ditches, however, it is not known yet whether they exhibit alternative stable states.

Based on competition theory and field data I found that ditches are unlikely to have alternative stable states (chapter 2). As a result, the forward transition from a state dominated by submerged plants to a state dominated by floating plants occurs at the same nutrient loading as the backward transition. For ecosystem management this means that it requires no extra reduction in nutrient loading to restore a ditch that became floating-plant dominated. This is in contrast to systems that do possess alternative stable states such as lakes, where the nutrient loading has to be reduced much further to restore the system.

Alternative stable states are often explained in terms of positive feedback loops; self-enhancing mechanisms which make a certain state persistent and hamper a transition to the contrasting state. Based on the constructed feedback diagram for ditches it seems likely that ditches have alternative states (Fig. 1.3b). However, chapter 2 points out that there is more nuance to it. It is not only about the presence of these feedback loops but also about their magnitude. In the case of submerged and floating plants it is important how much both plant suffer from each other. When both plants suffer too 
much from each other, they cannot coexist but instead keep each other out, leading to alternative stable states. This is the case when submerged plants consume too many nutrients for floating plants to persist, and when floating plants attenuate too much light for submerged plants to persist (Fig. 6.1, right panel). A different picture arises when the submerged plant has lower nutrient requirements than the floating plant. Then both plants suffer more from themselves than from the other, which results in coexistence (Fig. 6.1, left panel). This situation of coexistence is most plausible because submerged plants in general have lower nutrient demands than free-floating plants.
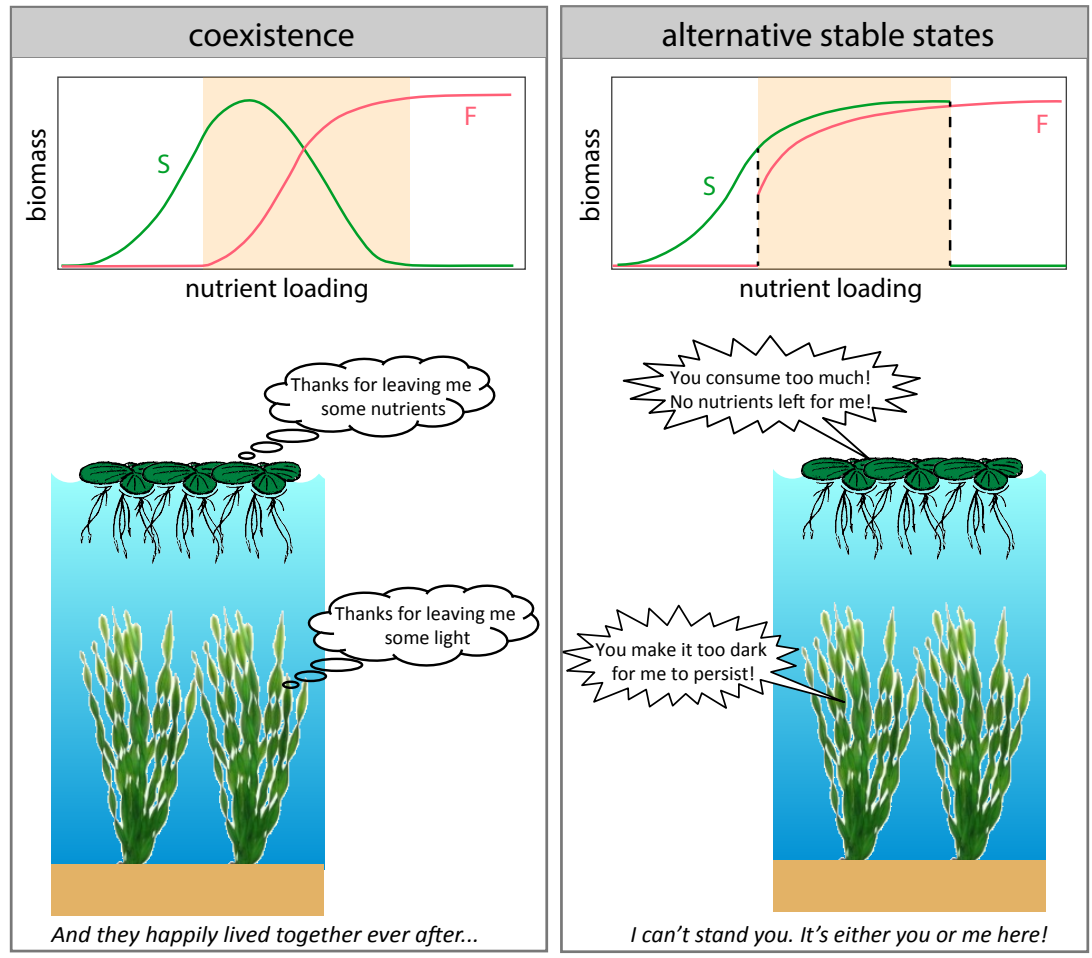

Figure 6.1. Cartoon illustrating how the chance on alternative stable states in ditches depends on the consumption traits of submerged plants compared to floating plants. The left situation (coexistence) is most likely given the consumption traits of common plants like water weed (Elodea) and duckweed (Lemna). Note that alternative stable states and coexistence are only relevant to ditches with intermediate nutrient loading (see shaded areas in upper panels where ' $F$ ' stands for floating plants and 'S' denotes submerged plants), as low nutrient loadings lead to dominance of submerged plants and high loadings cause floating-plant dominance. 
In chapter 2 I did not take into account that most submerged plants also take up nutrients from the sediment, instead of only from the water column. This sediment uptake might affect the chance on alternative stable states. I hypothesize that sediment uptake by submerged plants promotes coexistence, at least when the sediment is a separate source of nutrients, barely influencing the nutrient availability in the water column, because then both plants suffer less from each other's nutrient uptake from the water. On the other hand, sediment uptake might promote alternative stable states when the sediment and water column are a shared source of nutrients. Then, the floating plant suffers from the submerged plant's sediment nutrient uptake, especially when nutrients end up in a ditch via the sediment such that the submerged plants have priority to these nutrients.

Even if ditches would have alternative stable states, the question is to what extent this would hamper the restoration of floating-plant dominated ditches. One could argue that ditches are reset every year due to mowing or dredging, which breaks down the self-enhancing capacity of a state and therefore facilitates the restoration to the contrasting state.

\section{Restoring ditches by reducing nitrogen loading}

There is a long-standing debate in ecology about the effectiveness of reducing $\mathrm{N}$ loads to restore eutrophic ecosystems. Some argue that reducing $\mathrm{N}$ inputs favors species that fix $\mathrm{N}_{2}$ from the atmosphere and that these species provide enough $\mathrm{N}$ to correct for $\mathrm{N}$ deficits, which keeps the system P-limited and makes steering on $\mathrm{N}$ ineffective (Schindler et al. 2008). Others argue that these $\mathrm{N}_{2}$-fixers cannot meet the $\mathrm{N}$ requirements of the eutrophic ecosystem, implying that reducing $\mathrm{N}$ inputs would make sense (Lewis and Wurtsbaugh 2008). This debate mainly focusses on lakes. Less attention has been payed to floating plant-dominated systems such as ditches, which also harbor $\mathrm{N}_{2}$ fixing species such as the free-floating plant Azolla that lives in symbiosis with the $\mathrm{N}_{2}$-fixing cyanobacterium Anabaena azollae (Wagner 1997) (Fig. 6.2).

In this thesis I considered the competition between duckweed (Lemna) and water fern (Azolla) for light and nutrients to investigate whether the $\mathrm{N}_{2}$ fixing Azolla has the capacity to keep a eutrophic ditch P-limited when the ditch's $\mathrm{N}$ supply is lowered (chapter 3 ). Based on a resource competition model, an ecosystem model (PCDitch) and a 45-year set of field data (Limnodata Neerlandica) I found that it is unlikely that Azolla keeps a eutrophic ditch P-limited. This can be explained by the fact that $\mathrm{N}_{2}$-fixation comes with an energetic cost (Paerl 1990). As a result, $\mathrm{N}_{2}$-fixers are likely to have 
higher resource requirements and therefore cannot lower the $\mathrm{P}$ concentration enough to make eutrophic species like Lemna P-limited. Instead, the eutrophic ditch becomes N-limited, implying that Azolla cannot completely fulfil the $\mathrm{N}$ demands of the ditch's eutrophic species such as Lemna spp.

What are the consequences for management? The reduction of $\mathrm{N}$ inputs might still be unsuccessful because it can lead to the rise of Azolla, which has similar detrimental effects on the ditch ecology as Lemna. On the other hand, field data hint at constraints on $\mathrm{N}_{2}$-fixation that prevent Azolla from being abundant at low $\mathrm{N}$ availability (chapter 3). Such constraints include the limited availability of iron (Watanabe 1982), as iron-deficient Azolla plants can become yellow due to the depletion of chlorophyll and often have thin and whitish roots (Malavolta et al. 1981). Taking these constraints on $\mathrm{N}_{2}$-fixation into account, reducing the $\mathrm{N}$ loading to a ditch may pay off and lead to the return of submerged plants. The restoration success is probably even bigger when the reduction in $\mathrm{N}$ loading is combined with a reduction in $\mathrm{P}$ loading, given the high $\mathrm{P}$ demand of Lemna and Azolla in particular. This advocates a more balanced view on nutrient control, as promoted for other freshwater and coastal marine ecosystems (Conley et al. 2009), in which ecosystem management should not only focus on reducing $\mathrm{P}$ inputs but simultaneously focus on lowering $\mathrm{N}$ inputs.

The success of such a balanced nutrient approach to restore ditches will be system-specific. First of all the question arises to what extent the nutrient loading can be controlled. For example, some polder areas are subject to a high background nutrient loading due to upward seepage, which is difficult to control. In addition, most Dutch polder areas have a long history of intensive agriculture which led to a strong build up of nutrients (mainly P) in

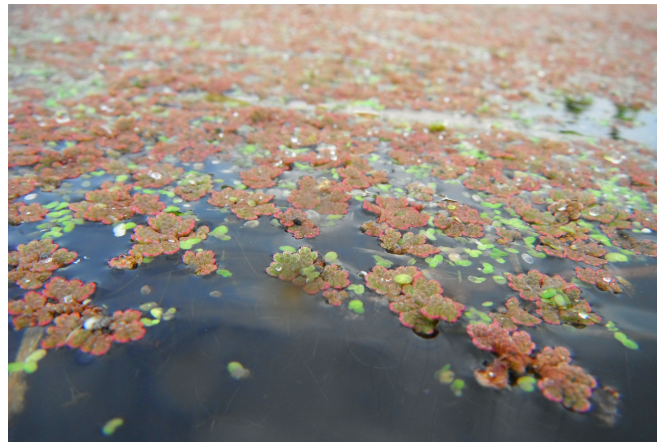

Figure 6.2. A ditch dominated by water fern (Azolla filiculoides) and duckweed (Lemna minor). Photo by Michiel Verhofstad. 
the soil (Oenema et al. 2005). This frustrates measures that aim at reducing nutrient loading. For example, it will take years before lowering the use of fertilizers in agriculture will have a considerable effect on the nutrient inputs to ditches (Schippers et al. 2006). Second, the restoration success will depend on the presence of environmental factors that prevent Azolla from becoming abundant. For example, restoration efforts will probably be more successful in areas without iron-rich upward seepage, such that the growth of Azolla will be inhibited by iron.

\section{A spatial view on ditches and their vulnerability to floating plants}

Paradoxically, most of our knowledge on the functioning of freshwater ecosystems such as lakes, ponds and ditches comes from studies on isolated systems, whereas freshwaters are often connected in a chain or network. Especially for ditches the spatial structure is evident, as ditches often make part of extensive networks (Fig. 6.3). We know from previous research on isolated ditches that a ditch switches to floating-plant dominance once a certain critical nutrient loading is exceeded (Portielje and Roijackers 1995) and that this critical nutrient loading depends on ditch characteristics such as water depth, sediment type and flow rate (Van Liere et al. 2007). But what about ditches in a network; is each ditch in the network equally vulnerable to floating-plant dominance or does the vulnerability depend on the ditch's position in the network?

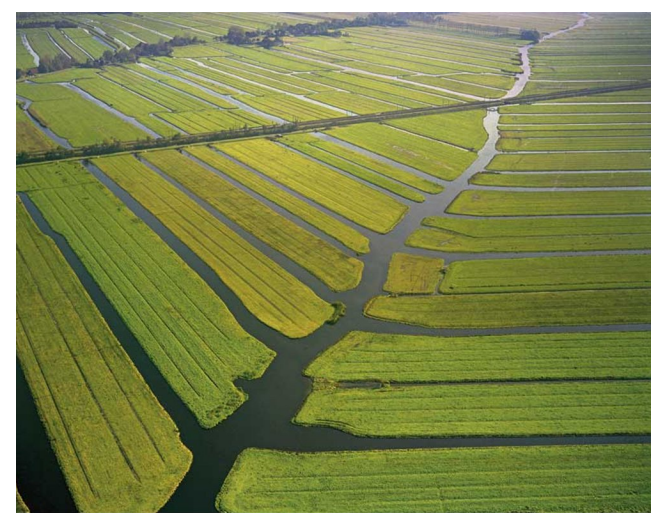

Figure 6.3. Typical network of ditches in a Dutch polder system.

To answer this question I considered the flow of water and nutrients throughout a network of ditches. Each ditch receives water and nutrients from the adjacent land by lateral runoff. These water and nutrients are transported to the polder outlet. Therefore, the flow of water and nutrients increases in 
the direction of the polder outlet.

I found that this increasing flow of water and nutrients does not automatically lead to spatial differences in ditch ecology (chapter 4). To be more specific, when looking solely at the increase of water and nutrient flow, so keeping other factors such as the ditches' water depth, sediment type and lateral runoff the same, both a simple and a complex model (PCDitch) predict that all ditches eventually have the same ecological state and therefore are equally vulnerable to a regime shift. A different picture arises however when there are spatial differences in ditch characteristics (width, depth, sediment type, etc.) or lateral runoff. Then, the ecosystem state differs throughout the network and the chance on floating-plants depends on the ditch's position in the network.

For ecosystem management this implies that the amount of heterogeneity within a polder is of big importance. In a rather homogeneous polder, in terms of its ditches (width, depth, sediment type, etc.) and its land use (enabling similar lateral runoff to the ditches), the vulnerability to floating-plant dominance, similar for all ditches, can already be predicted with a non-spatial model that only considers the local input of water and nutrients by lateral runoff. In a more heterogeneous polder, the water and nutrient loading from upstream ditches can no longer be ignored. As a consequence, you need a spatial model to predict the vulnerability to floating plants. This vulnerability differs per ditch, which is in agreement with the observed spatial heterogeneity in most polders, where some ditches are completely covered by floating plants while others are not. This complicates the complete recovery of a polder network to submerged plants, and promotes a more spatial view on ecosystem management in which restoration efforts should focus on certain polder parts that are most promising for recovery.

For this research I applied the ecosystem model PCDitch in a spatial context. I did this by coupling PCDitch to the hydrodynamic model SOBEK (Delft Hydraulics 1996). This PCDitch-SOBEK model breaks new grounds for exploring spatial management strategies. For example, the effect of extensifying agriculture or constructing nature areas can be explored; where should such areas be located to maximize the ecological quality of ditches? And is it beneficial for the ditch ecology to enlarge the path by which water flows to the polder outlet by constructing flow barriers, and where should these barriers be placed to maximize their effect? And what are the ecological consequences of less intensive mowing, and how does it affect the discharge capacity and flooding risk of the ditches? 


\subsection{Future challenges and opportunities}

The world is changing in a rapid pace. Whereas the planet's environment used to change in a slow and natural manner over the past millions of years, nowadays it changes increasingly fast due to human activities, which have become the main driver of environmental change (Rockstrom et al. 2009). Fig. 6.4 shows current state of environmental change. For some environmental changes such as climate change and biodiversity loss, the critical thresholds have already been overstepped implying that the caused damage might be irreversible. These ongoing environmental changes put pressure on the ecosystems of the planet. The exact ecological impact is yet to be understood, and its understanding is complicated by the co-occurrence of multiple changes, such as simultaneous changes in climate, land use and nutrient cycling.

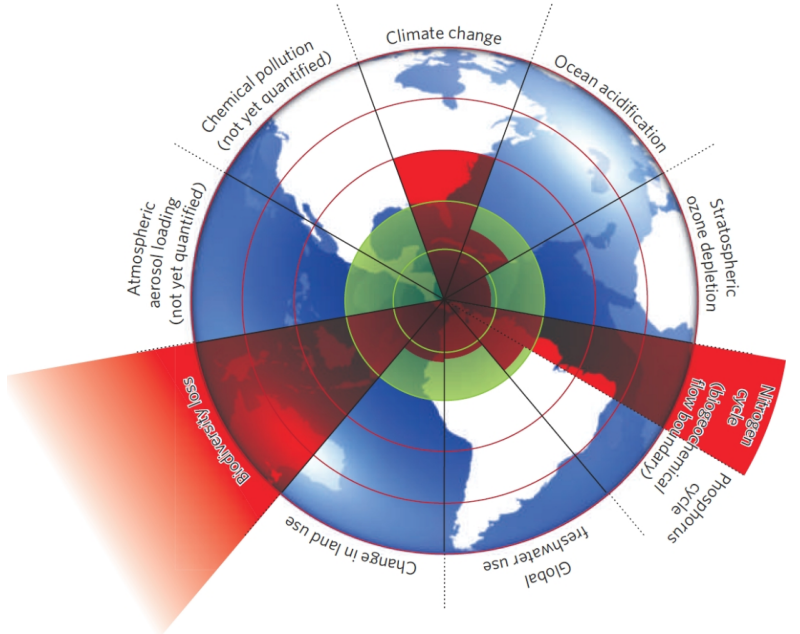

Figure 6.4. The current state of the world (red wedges) with respect to environmental change, where the inner green circle represents the critical thresholds or safe operating space beyond which the changes might be irreversible. This figure is taken from Rockstrom et al. (2009).

In the light of these future changes and the challenges it brings about, the methods used and developed in this thesis help to better understand, predict and anticipate the impact of environmental changes on the ecology of freshwater ecosystems like ditches. I highlight the method of combining models of different complexity, and the future opportunities with respect to spatial ecosystem modelling. 


\section{Combining models of different complexity}

The development and use of models with different complexity plays an important role in this thesis (Fig 1.6). I combined simple and complex models in two ways. First, I used complex models to see if the insights generated with the simple models hold true at the ecosystem level. I did this in chapter 3 by embedding the simple resource competition model on Azolla and Lemna in the ecosystem model PCDitch. Second, I used simple models to better understand the behavior of complex models. This was done in chapter 4 , where the puzzling result, predicted by PCDitch, that connected ditches are equally vulnerable to a regime shift could be explained with a simple model on the water and nutrient fluxes in connected ditches.

These examples illustrate that combining models of different complexity allows you to combine the explanatory strength of simple models and the predictive power of complex models. This can be seen as a way to bridge the gap between fundamental and applied ecology. Fundamental ecology strongly leans on simple models, as much ecological theory is developed or underpinned by simple models (Grimm 1994). Applied ecology deals with ecological issues and management options at the ecosystem level and therefore strongly relies on the predictive power of complex ecosystem models. Therefore, combining simple and complex models brings the essentially different worlds of fundamental and applied ecology closer to each other.

Switching in model complexity may also optimize the scientific gain of your research. This is illustrated by the 'Medawar Zone' showing the trade-off between the complexity of a question and the scientific reward that is obtained when you solve the question (Fig. 6.5). The Medawar Zone is called

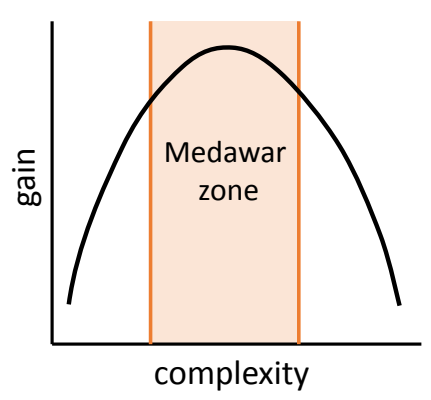

Figure 6.5. The 'Medawar zone' indicating that solving problems of intermediate complexity generates the highest scientific reward. This figure is modified from Loehle (1990). 
after Sir Peter Medawar, a Nobel prize-winning medical researcher, who characterized science as the 'art of soluble' (Medawar 1967). According to the Medawar zone, it pays off most to solve questions of intermediate complexity, as solving easy questions may be either too trivial or too well within reach, whereas difficult questions may be not solvable at all because the associated tools and technology are not advanced enough or because the solution is too difficult to verify (Loehle 1990). The concept of the Medawar zone can be translated to models, in a sense that there is a certain model complexity at which a model produces the most valuable and fruitful insights. Switching in model complexity can be used to find this optimal model complexity.

There are a couple of recent technological advances that facilitate the development of models with different complexity. For example, the Framework for Aquatic Biogeochemical Models (FABM) enables the coupling of an ecological model to a variety of 0D-3D hydrodynamic models (Bruggeman and Bolding 2014). Combined with the fact that the ecological model can be built up from different ecological modules, FABM provides great flexibility to develop models with different complexity and with different spatial extent. A similar initiative is Delwaq (Delft Hydraulics 1995), which just like FABM acts as a communicator between hydrodynamic and ecological models. Another recent technological development in modelling is the Database Approach To Modelling (DATM) (Mooij et al. 2014). The philosophy behind DATM is somewhat different to that of FABM and Delwaq, in a sense that FABM and Delwaq can be regarded as single 'super-frameworks' incorporating multiple models, whereas DATM focusses on a single model and its implementation in multiple frameworks. DATM stores a model in a clear and framework-independent way by using a database-format. This setup proved to be very convenient for developing and constructing models with different complexity, and therefore was used in this thesis for that purpose.

Concluding, combining models of different complexity, as supported by the technological advances mentioned above, has the potential to bring scientists, model-users and ecosystem managers closer to each other, allowing for a healthy cross-fertilization of expertise and ideas between these different disciplines. 


\section{Spatial ecosystem modeling}

Given recent technological advances, there are growing opportunities for spatial ecosystem modeling. First of all, there are developments such as Delwaq and FABM that facilitate the coupling of ecological models to hydrodynamic models, such that the ecology of connected aquatic systems can be regarded in a spatial context. An example of this is the coupling between PCDitch and SOBEK, as established in this thesis. Second, spatial ecosystem modeling, which is quite demanding in terms of computational power, clearly benefits from computers becoming faster and faster. This increase in speed is marked by the number of transistors in a CPU that has doubled approximately every two years since the 70's, a phenomenon referred to as Moore's law (Schaller 1997). Third, the growing amount of remote sensing data with a high spatial coverage from satellites and drones can be used to feed and validate spatial ecosystem models. Such data can be used for example to deduce the spatial coverage of water plants or the chlorophyll contents of surface waters.

Due to these developments, the role of spatial ecosystem modeling in supporting ecosystem management is likely to become more prominent. Especially for ditches this is a promising development, given the fact that ditches are highly connected. Figure 6.6 illustrates the future opportunities of spatial ecosystem modelling for ditches. It shows how the ecology of ditches can be simulated at the polder scale by running an ecosystem model like PCDitch coupled to a hydrodynamic model, fed or validated with spatial data from satellites or drones.

The setup of such a spatial model system can be made even more sophisticated. For example, the model system can be extended with models that describe the transport of water and nutrients from the adjacent land to the ditch via the groundwater and the unsaturated zone. Such models can, in turn, be coupled to meteorological models in order to construct an integrated multi-disciplinary model system that gives an encompassing view on the hydrology and ecology of ditches at the polder scale. It is even conceivable that such an integrated model system runs on a real-time basis. This would allow for continuous real-time calibration or validation of the model system with remote sensing data, potentially enabling short-term predictions of the ditch ecology, similar to the weather forecast.

Besides all future opportunities with regard to spatial ecosystem modeling, there are also quite some challenges ahead. One of the most challenging aspects is how to deal with the movement of biota. Think for example of free-floating plants like duckweed. Their transport is difficult to simulate, as 




Figure 6.6. Future opportunities with respect to spatial ecosystem modelling. How spatial data of satellites and drones can be used to feed and calibrate spatial ecosystem models, allowing for real-time validation and short-term predictions of the ecology of ditches.

these plants are carried by the water flow only partly and their transport is also affected by wind and flow obstructions like culverts or weirs. It becomes even more complicated when modeling the transport of organisms that move actively, such as macro-invertebrates or fish.

Another ongoing challenge in spatial ecosystem modeling, although already tackled in some spatial modeling environments like Duflow (Aalderink et al. 1995) and FABM, is to incorporate the feedback of ecology on hydrodynamics. A well-known example of such a feedback is the effect of submerged plants on the water flow (Järvelä 2002). The flow obstruction by submerged plants can lead, especially in times of high discharges, to higher water levels and even to flooding. Accounting for the feedback of biota on hydrodynamics requires to extend the information transfer between ecological and hydrodynamic models from the common one-way transfer, from hydrodynamics 
to ecology, to a two-way communication in which the ecological model also transfers information to the hydrodynamic model. Such a two-way transfer requires both models to exchange information during the model run and thus to be run simultaneously.

\section{Concluding remarks}

Although nature is too complex to fully understand, I envision that the further development of the concepts and techniques described in this thesis such as spatial ecosystem modeling and combining models of different complexity will help us to step by step unravel the complex functioning of nature. The better we know how our ecosystems function, the better we can protect them against the ongoing human-driven environmental changes. In this way we can contribute to making the planet's ecosystems future-proof in order to preserve them for future generations. 


\section{References}

Aalderink, R. H., N. J. Klaver, and R. Noorman. 1995. Duflow V 2.0 Microcomputer package for the simulation of 1-dimensional flow and water quality in a network of open water courses.

Agawin, N. S., S. Rabouille, M. J. Veldhuis, L. Servatius, S. Hol, H. M. van Overzee, and J. Huisman. 2007. Competition and facilitation between unicellular nitrogen-fixing cyanobacteria and non-nitrogen-fixing phytoplankton species. Limnology and Oceanography 52:2233-2248.

Ahearn, M., M. L. Pace and P. M. Groffman. 2013. Successes, limitations, and frontiers in ecosystem science, Springer Science \& Business Media.

Ahlgren, I. 1980. A dilution model applied to a system of shallow eutrophic lakes after diversion of sewage effluents. Archiv für Hydrobiologie 89:1732.

Aldenberg, T., J. H. Janse and P. R. M. Kramer. 1995: Fitting the dynamic model PCLake to a multi-lake survey through Bayesian Statistics. Ecological Modelling 78: 83-99.

Anderson, D. M., P. M. Glibert, and J. M. Burkholder. 2002. Harmful algal blooms and eutrophication: nutrient sources, composition, and consequences. Estuaries 25:704-726.

Argent, R. M., 2004. An overview of model integration for environmental applications - components, frameworks and semantics. Environmental Modelling \& Software 19: 219-234.

Armitage, P. D., K. Szoszkiewicz, J. H. Blackburn, and I. Nesbitt. 2003. Ditch communities: a major contributor to floodplain biodiversity. Aquatic Conservation: Marine and Freshwater Ecosystems 13:165-185.

Barko, J. W., D. Gunnison, and S. R. Carpenter. 1991. Sediment interactions with submersed macrophyte growth and community dynamics. Aquatic Botany 41:41-65.

Bel, G., A. Hagberg, and E. Meron. 2012. Gradual regime shifts in spatially extended ecosystems. Theoretical Ecology 5:591-604. 
Berman-Frank, I., A. Quigg, Z. V. Finkel, A. J. Irwin, and L. Haramaty. 2007. Nitrogen-fixation strategies and Fe requirements in cyanobacteria. Limnology and Oceanography 52:2260-2269.

Bieleski, R. L., and A. Lauchli. 1992. Phosphate-uptake, efflux and deficiency in the water fern, Azolla. Plant Cell and Environment 15: 665-673.

Brendonck, L., J. Maes, W. Rommens, N. Dekeza, T. Nhiwatiwa, M. Barson, V. Callebaut et al. 2003. The impact of water hyacinth (Eichhornia crassipes) in a eutrophic subtropical impoundment (Lake Chivero, Zimbabwe). II. Species diversity. Archiv für Hydrobiologie 158:389-405.

Broers, M., 2012. Effect of climate change on the ecology of a ditch. BSc. Thesis. Wageningen University and Research Centre, Wageningen, The Netherlands.

Bruggeman, J., and K. Bolding. 2014. A general framework for aquatic biogeochemical models. Environmental Modelling \& Software 61:249-265.

Carpenter, S. R., N. F. Caraco, D. L. Correll, R. W. Howarth, A. N. Sharpley, and V. H. Smith. 1998. Nonpoint pollution of surface waters with phosphorus and nitrogen. Ecological Applications 8:559-568.

Carpenter, S., and R. Lathrop. 2014. Phosphorus loading, transport and concentrations in a lake chain: a probabilistic model to compare management options. Aquatic Sciences 76:145-154.

Caswell, H., 1989: Matrix population models. Wiley Online Library.

Chesson, P. 2000. Mechanisms of Maintenance of Species Diversity. Annual Review of Ecology and Systematics 31:343-366.

Chow, S. N., Mallet-Paret, J. and E.S. van Vleck. 1996. Dynamics of lattice differential equations. International Journal of Bifurcation and Chaos 6: 1605-1621.

Clemmens, A., Holly, F. and W. Schuurmans. 1993: Description and Evaluation of Program: Duflow. Journal of Irrigation and Drainage Engineering 119: 724-734.

Conley, D. J., H. W. Paerl, R. W. Howarth, D. F. Boesch, S. P. Seitzinger, K. E. Havens, C. Lancelot et al. 2009. Controlling eutrophication: nitrogen and phosphorus. Science 323:1014-1015.

Costa, M. L., M. C. Santos, and F. Carrapiço. 1999. Biomass characterization of Azolla filiculoides grown in natural ecosystems and wastewater. Hydrobiologia 415:323-327.

Cottenie, K., E. Michels, N. Nuytten, and L. de Meester. 2003. Zooplankton metacommunity structure: regional vs. local processes in highly interconnected ponds. Ecology 84:991-1000. 
David, O., J. C. Ascough, I., Lloyd, W., Green, T. R., Rojas, K. W., Leavesley, G. H. and L.R. Ahuja. 2013. A software engineering perspective on environmental modeling framework design: The Object Modeling System. Environmental Modelling \& Software 39: 201-213.

De Boer, R. and L. Pagie. 1983. GRIND: Great integrator differential equations. Bioinformatics Group, University of Utrecht, The Netherlands.

De Nobel, W. T., J. Huisman, J. L. Snoep, and L. R. Mur. 1997. Competition for phosphorus between the nitrogen-fixing cyanobacteria Anabaena and Aphanizomenon, v. 24.

De Roos, A. M. and L. Persson. 2012. Population and community ecology of ontogenetic development. Princeton University Press.

De Tezanos Pinto, P., and I. O'Farrell. 2014. Regime shifts between free-floating plants and phytoplankton: a review. Hydrobiologia 740:13-24.

Delft Hydraulics 1995. DELWAQ version 4.0 Technical Reference Manual. Delft, The Netherlands.

Delft Hydraulics 1996. Sobek Users Guide, Version 1.10; Delft Hydraulics \& RWS-RIZA, Delft, The Netherlands.

Deltares, 2013: D-Water Quality, Water quality and aquatic ecology modelling suite. User Manual - Water Quality and Aquatic Ecology, and Technical Reference Manual - Processes Library Description. Delft, The Netherlands: Deltares.

Dhooge, A., Govaerts, W. and Y. A. Kuznetsov. 2003. MATCONT: a MATLAB package for numerical bifurcation analysis of ODEs. ACM Transactions on Mathematical Software 29: 141-164.

Doedel, E. J., and B. Oldeman. 2009. Auto 07p: Continuation and bifurcation software for ordinary differential equations, Technical report. Concordia University, Montreal, Canada.

Donchyts, G. and B. Jagers. 2010. DeltaShell - an open modelling environment. In: International Environmental Modelling and Software Society 2010 International Congress on Environmental Modelling and Software.

Dublin, H. T., A. R. E. Sinclair, and J. McGlade. 1990. Elephants and Fire as Causes of Multiple Stable States in the Serengeti-Mara Woodlands. Journal of Animal Ecology 59:1147-1164.

EC-JRC-ISIS. 2002. SimLab v. 2.2, User manual. European Commission, Joint Research Centre, Inst. for Systems, Informatics and Safety, Ispra, Italy.

Elser, J. J., M. E. Bracken, E. E. Cleland, D. S. Gruner, W. S. Harpole, H. Hillebrand, J. T. Ngai et al. 2007. Global analysis of nitrogen and phosphorus limitation of primary producers in freshwater, marine and terrestrial 
ecosystems. Ecology Letters 10:1135-1142.

Elzinga, J., 2013. Projected Social and Ecological Impacts of Marker Wetlands Projects on the Lake Markermeer Region. MSc. thesis. Wageningen University and Research Centre, Wageningen, The Netherlands.

Ferber, L., S. Levine, A. Lini, and G. Livingston. 2004. Do cyanobacteria dominate in eutrophic lakes because they fix atmospheric nitrogen? Freshwater Biology 49:690-708.

Feuchtmayr, H., R. Moran, K. Hatton, L. Connor, T. Heyes, B. Moss, I. Harvey et al. 2009. Global warming and eutrophication: effects on water chemistry and autotrophic communities in experimental hypertrophic shallow lake mesocosms. Journal of Applied Ecology 46:713-723.

Fisher, M. M., S. J. Miller, A. D. Chapman, and L. W. Keenan. 2009. Phytoplankton dynamics in a chain of subtropical blackwater lakes: the Upper St. Johns River, Florida, USA. Lake and Reservoir Management 25:73-86.

Forchhammer, N. C. 1999. Production potential of aquatic plants in systems mixing floating and submerged macrophytes. Freshwater Biology 41:183191.

Forrester, J. W., 1961. Industrial dynamics 2. MIT press Cambridge, MA.

Fox, J., 2009. Aspects of the Social Organization and Trajectory of the R Project. The R Journal 1: 5-13.

Gerla, D. J., W. M. Mooij, and J. Huisman. 2011. Photoinhibition and the assembly of light-limited phytoplankton communities. Oikos 120:359368.

Grant, H. and C.K. Lai. 1998. Simulation modeling with artificial reality technology (SMART): an integration of virtual reality and simulation modeling. In: Simulation Conference Proceedings, 1998. Winter 1. IEEE, pp. 437-441.

Grimm, V. 1994. Mathematical models and understanding in ecology. Ecological Modelling 75:641-651.

Hare, S. R., and N. J. Mantua. 2000. Empirical evidence for North Pacific regime shifts in 1977 and 1989. Progress In Oceanography 47:103-145.

Hecky, R. E., and P. Kilham. 1988. Nutrient Limitation of Phytoplankton in Freshwater and Marine Environments: A Review of Recent Evidence on the Effects of Enrichment. Limnology and Oceanography 33:796-822.

Heisler, J., P. M. Glibert, J. M. Burkholder, D. M. Anderson, W. Cochlan, W. C. Dennison, Q. Dortch et al. 2008. Eutrophication and harmful algal blooms: a scientific consensus. Harmful algae 8:3-13.

Higler, L., and P. Verdonschot. 1989. Macroinvertebrates in the Demmerik 
ditches (The Netherlands): The role of environmental structure. Aquatic Ecology 23:143-150.

Hillbricht-Ilkowska, A. 2002. Eutrophication rate of lakes in the Jorka river system (Masurian Lakeland, Poland): long term changes and trophic correlations. Polish Journal of Ecology 50:475-487.

Hilt, S., J. Köhler, H.-P. Kozerski, E. H. van Nes, and M. Scheffer. 2011. Abrupt regime shifts in space and time along rivers and connected lake systems. Oikos 120:766-775.

Howarth, R. W., R. Marino, J. Lane, and J. J. Cole. 1988. Nitrogen fixation in freshwater, estuarine, and marine ecosystems. 1. Rates and importance. Limnology and Oceanography 33:669-687.

Huisman, J., and F. J. Weissing. 1994. Light-Limited Growth and Competition for Light in Well-Mixed Aquatic Environments: An Elementary Model. Ecology 75:507-520.

Huisman, J., and F. J. Weissing. 1995. Competition for Nutrients and Light in a Mixed Water Column: A Theoretical Analysis. The American Naturalist 146:536-564.

Huisman, J., P. v. Oostveen, and F. J. Weissing. 1999a. Species Dynamics in Phytoplankton Blooms: Incomplete Mixing and Competition for Light. The American Naturalist 154:46-68.

Huisman, J., R. R. Jonker, C. Zonneveld, and F. J. Weissing. 1999b. Competition for Light between Phytoplankton Species: Experimental Tests of Mechanistic Theory. Ecology 80:211-222.

Huisman, J., N. N. Pham Thi, D. M. Karl, and B. Sommeijer. 2006. Reduced mixing generates oscillations and chaos in the oceanic deep chlorophyll maximum. Nature 439:322-325.

Jaarsma, N., M. Klinge, and L. P. M. Lamers. 2008. Een ecologische systeemanalyse en diagnose van ondiepe meren en plassen voor de Kaderrichtlijn Water: Van helder naar troebel... En weer terug. Utrecht, STOWA.

Jachner, S., van den Boogaart, G. and T. Petzoldt. 2007. Statistical methods for the qualitative assessment of dynamic models with time delay (R Package qualV). Journal of Statistical Software 22: 1-31.

Jäger, C. G., and S. Diehl. 2014. Resource competition across habitat boundaries: asymmetric interactions between benthic and pelagic producers. Ecological Monographs 84:287-302.

Janes, R., J. Eaton, and K. Hardwick. 1996. The effects of floating mats of Azolla filiculoides Lam. and Lemna minuta Kunth on the growth of submerged macrophytes. Hydrobiologia 340:23-26. 
Janse, J. H. 1997. A model of nutrient dynamics in shallow lakes in relation to multiple stable states. Hydrobiologia 342:1-8.

Janse, J. H. 1998. A model of ditch vegetation in relation to eutrophication. Water Science and Technology 37:139-149.

Janse, J. H. and P. J. T. M. van Puijenbroek, 1998: Effects of eutrophication in drainage ditches. Environmental Pollution 102: 547-552.

Janse, J. H., Van Donk, E. and T. Aldenberg. 1998. A model study on the stability of the macrophyte-dominated state as affected by biological factors. Water Research 32: 2696-2706.

Janse, J. H., Ligtvoet, W., Van Tol, S. and A. H. M. Bresser. 2001. A model study on the role of wetland zones in lake eutrophication and restoration. The Scientific World Journal 1: 605-614.

Janse, J. H. 2005. Model studies on the eutrophication of shallow lakes and ditches. PhD Thesis, Wageningen University, Wageningen.

Janse, J. H., L. N. De Senerpont Domis, M. Scheffer, L. Lijklema, L. Van Liere, M. Klinge, and W. M. Mooij. 2008. Critical phosphorus loading of different types of shallow lakes and the consequences for management estimated with the ecosystem model PCLake. Limnologica-Ecology and Management of Inland Waters 38:203-219.

Janse, J. H., M. Scheffer, L. Lijklema, L. Van Liere, J. S. Sloot, and W. M. Mooij. 2010. Estimating the critical phosphorus loading of shallow lakes with the ecosystem model PCLake: Sensitivity, calibration and uncertainty. Ecological Modelling 221:654-665.

Janssen, A. B. G., G. Arhonditsis, A. Beusen, K. Bolding, L. Bruce, J. Bruggeman, R.-M. Couture et al. 2015. Exploring, exploiting and evolving diversity of aquatic ecosystem models: a community perspective. Aquatic Ecology 49: 513-548.

Järvelä, J. 2002. Flow resistance of flexible and stiff vegetation: a flume study with natural plants. Journal of Hydrology 269:44-54.

Jeppesen, E., M. Søndergaard, B. Kronvang, J. P. Jensen, L. M. Svendsen, and T. L. Lauridsen. 1999. Lake and catchment management in Denmark, Pages 419-432 The Ecological Bases for Lake and Reservoir Management, Springer.

Joppa, L. N., McInerny, G., Harper, R., Salido, L., Takeda, K., O’Hara, K., Gavaghan, D. and S. Emmott. 2013. Troubling Trends in Scientific Software Use. Science 340: 814-815.

Kirk, J. T. O. 1994. Light and photosynthesis in aquatic ecosystems, Cambridge university press. 
Klepper, O., 1989. A model of carbon flows in relation to macrobenthic food supply in the Oosterschelde estuary (S.W. Netherlands). PhD Dissertation, LUW Wageningen The Netherlands, $270 \mathrm{pp}$.

Klepper, O., 1997. Multivariate aspects of model uncertainty analysis: tools for sensitivity analysis and calibration. Ecological Modelling 101: 1-13.

Knowlton, N. 1992. Thresholds and Multiple Stable States in Coral Reef Community Dynamics. American Zoologist 32:674-682.

Kooi, B., 2003. Numerical bifurcation analysis of ecosystems in a spatially homogeneous environment. Acta biotheoretica 51: 189-222.

Krause-Jensen, D., and K. Sand-Jensen. 1998. Light Attenuation and Photosynthesis of Aquatic Plant Communities. Limnology and Oceanography 43:396-407.

Lewis, W. M., and W. A. Wurtsbaugh. 2008. Control of lacustrine phytoplankton by nutrients: erosion of the phosphorus paradigm. International Review of Hydrobiology 93:446-465.

Lewis, W. M., W. A. Wurtsbaugh, and H. W. Paerl. 2011. Rationale for control of anthropogenic nitrogen and phosphorus to reduce eutrophication of inland waters. Environmental Science \& Technology 45:10300-10305.

Lischke, B., Hilt, S., Janse, J. H., Kuiper, J. J., Mehner, T., Mooij, W. M. and U. Gaedke. 2014. Enhanced Input of Terrestrial Particulate Organic Matter Reduces the Resilience of the Clear-Water State of Shallow Lakes: A Model Study. Ecosystems 17: 616-626.

Lloyd, W., David, O., Ascough II, J., Rojas, K., Carlson, J., Leavesley, G., Krause, P., Green, T. and L. Ahuja. 2011. Environmental modeling framework invasiveness: Analysis and implications. Environmental Modelling \& Software 26: 1240-1250.

Loehle, C. 1990. A guide to increased creativity in research: inspiration or perspiration? Bioscience 40:123-129.

Lotka, A. J. 1920. Undamped oscillations derived from the law of mass action. Journal of the American chemical society 42:1595-1599.

Lu, J., Z. Wang, W. Xing, and G. Liu. 2013. Effects of substrate and shading on the growth of two submerged macrophytes. Hydrobiologia 700:157-167.

Madsen, T. V., and N. Cedergreen. 2002. Sources of nutrients to rooted submerged macrophytes growing in a nutrient-rich stream. Freshwater Biology 47:283-291.

Mäkelä, S., E. Huitu, and L. Arvola. 2004. Spatial patterns in aquatic vegetation composition and environmental covariates along chains of lakes in the Kokemäenjoki watershed (S. Finland). Aquatic Botany 80:253-269. 
Malavolta, E., W. Acorsi, A. Ruschel, F. Krug, L. Nakayama, and I. Eimori. 1981. Mineral nutrition and N2-fixation in Azolla. Associative N2-fixation/editors, Peter B. Vose, Alaides P. Ruschel.

Martin, S. L., and P. A. Soranno. 2006. Lake landscape position: relationships to hydrologic connectivity and landscape features. Limnology and Oceanography 51:801-814.

Medawar, P. B. 1967. The art of the soluble.

Mehra, A., M. Farago, D. Banerjee, and K. Cordes. 1999. The water hyacinth: an environmental friend or pest? A review. Resource and environmental biotechnology 2:255-281.

Mieleitner, J. and Reichert, P., 2006: Analysis of the transferability of a biogeochemical lake model to lakes of different trophic state. Ecological Modelling 194: 49-61.

Mitchell, E. E. and Gauthier, J. S., 1976: Advanced continuous simulation language (ACSL). Simulation 26: 72-78.

Mitchell \& Gauthier Associates, 1995: Advanced continuous simulation language (ACSL): reference manual, Version 11. MGA Software.

Mooij, W. M. and Boersma, M., 1996: An object-oriented simulation framework for individual-based simulations (OSIRIS): Daphnia population dynamics as an example. Ecological Modelling 93: 139-153.

Mooij, W. M., Janse, J. H., De Senerpont Domis, L. N., Hülsmann, S. and B.W. Ibelings. 2007. Predicting the effect of climate change on temperate shallow lakes with the ecosystem model PCLake. In: Shallow Lakes in a Changing World. Springer, pp. 443-454.

Mooij, W. M., De Senerpont Domis, L. N. and J.H. Janse. 2009. Linking speciesand ecosystem-level impacts of climate change in lakes with a complex and a minimal model. Ecological Modelling 220: 3011-3020.

Mooij, W.M., Trolle, D., Jeppesen, E., Arhonditsis, G., Belolipetsky, P.V., Chitamwebwa, D.B.R., Degermendzhy, A.G. et al. 2010. Challenges and opportunities for integrating lake ecosystem modelling approaches. Aquatic Ecology 44: 633-667.

Mooij, W. M., R. J. Brederveld, J. J. de Klein, D. L. DeAngelis, A. S. Downing, M. Faber, D. J. Gerla et al. 2014. Serving many at once: How a database approach can create unity in dynamical ecosystem modelling. Environmental Modelling \& Software 61:266-273.

Moretti, A., and G. Siniscalco Gigliano. 1988. Influence of light and pH on growth and nitrogenase activity on temperate-grown Azolla. Biology and Fertility of Soils 6:131-136. 
Morris, M. D., 1991. Factorial sampling plans for preliminary computational experiments. Technometrics 33: 161-174.

Morris, K., K. A. Harrison, P. C. Bailey, and P. I. Boon. 2004. Domain shifts in the aquatic vegetation of shallow urban lakes: the relative roles of low light and anoxia in the catastrophic loss of the submerged angiosperm Vallisneria americana. Marine and Freshwater Research 55:749-758.

Muetzelfeldt, R. and J. Massheder. 2003. The Simile visual modelling environment. European Journal of Agronomy 18: 345-358.

Netten, J. J. C., G. H. P. Arts, R. Gylstra, E. H. van Nes, M. Scheffer, and R. M. M. Roijackers. 2010. Effect of temperature and nutrients on the competition between free-floating Salvinia natans and submerged Elodea nuttallii in mesocosms. Fundamental and Applied Limnology 177:125-132.

Nielsen, A., Trolle, D., Bjerring, R., Søndergaard, M., Olesen, J. E., Janse, J. H., Mooij, W. M. and E. Jeppesen. 2014. Effects of climate and nutrient load on the water quality of shallow lakes assessed through ensemble runs by PCLake. Ecological Applications 24: 1926-1944.

Oenema, O., L. van Liere, and O. Schoumans. 2005. Effects of lowering nitrogen and phosphorus surpluses in agriculture on the quality of groundwater and surface water in the Netherlands. Journal of Hydrology 304:289301.

Oliver, J. D. 1993. A review of the biology of giant salvinia. Faculty Publications and Presentations:87.

O'Neill, R. V. and R.H. Gardner. 1979. Sources of uncertainty in ecological models. In: B. P. Zeigler, M. S. Elzas, G. J. Kliv, T. I. Oren (eds.), Methodology in Systems Modelling and Simulation. p. 447-463. North-Holland Publishing Co.

Paerl, H. W. 1990. Physiological ecology and regulation of N2 fixation in natural waters, Pages 305-344 Advances in microbial ecology, Springer.

Paerl, H. W., and J. T. Scott. 2010. Throwing fuel on the fire: Synergistic effects of excessive nitrogen inputs and global warming on harmful algal blooms. Environmental science \& technology 44:7756-7758.

Paerl, H. W., H. Xu, N. S. Hall, G. Zhu, B. Qin, Y. Wu, K. L. Rossignol et al. 2014. Controlling cyanobacterial blooms in hypertrophic Lake Taihu, china: will nitrogen reductions cause replacement of non-N2 fixing by N2 fixing taxa? PloS one 9:e113123.

Passarge, J., S. Hol, M. Escher, and J. Huisman. 2006. Competition for nutrients and light: stable coexistence, alternative stable states, or competitive exclusion? Ecological Monographs 76:57-72. 
Perry, L. G., C. Neuhauser, and S. M. Galatowitsch. 2003. Founder control and coexistence in a simple model of asymmetric competition for light. Journal of Theoretical Biology 222:425-436.

Petzoldt, T. and K. Rinke. 2007. Simecol: an object-oriented framework for ecological modeling in R. Journal of Statistical Software 22: 1-31.

Portielje, R., and R. M. M. Roijackers. 1995. Primary succession of aquatic macrophytes in experimental ditches in relation to nutrient input. Aquatic Botany 50:127-140.

Press, W. H., Teukolsky, S. A., Vetterling, W. T. and B.P. Flannery. 2009. Numerical recipes in C+: the art of scientific computing 994. Cambridge University Press Cambridge.

R Core Team. 2013. R: A language and environment for statistical computing. R Foundation for Statistical Computing, Vienna, Austria. URL: http://www.R-project.org/.

Refsgaard, J. C. and H.J. Henriksen. 2004: Modelling guidelines - terminology and guiding principles. Advances in Water Resources 27: 71-82.

Richmond, B. and S. Peterson. 1985. Stella. Lyme, NH: High Performance Systems.

Rietkerk, M., F. v. d. Bosch, and J. v. d. Koppel. 1997. Site-Specific Properties and Irreversible Vegetation Changes in Semi-Arid Grazing Systems. Oikos 80:241-252.

Rockstrom, J., W. Steffen, K. Noone, A. Persson, F. S. Chapin, E. F. Lambin, T. M. Lenton et al. 2009. A safe operating space for humanity. Nature 461:472-475.

Roijackers, R., Szab, oacute, S., and M. Scheffer. 2004. Experimental analysis of the competition between algae and duckweed. Archiv für Hydrobiologie 160:401-412.

Ryabov, A. B., L. Rudolf, and B. Blasius. 2010. Vertical distribution and composition of phytoplankton under the influence of an upper mixed layer. Journal of Theoretical Biology 263:120-133.

Ryabov, A. B., and B. Blasius. 2011. A graphical theory of competition on spatial resource gradients. Ecology Letters 14:220-228.

Ryabov, A. 2012. Phytoplankton competition in deep biomass maximum. Theoretical Ecology 5:373-385.

Rykiel, E. J. 1996. Testing ecological models: the meaning of validation. Ecological modelling 90:229-244.

Saltelli, A., Ratto, M., Andres, T., Campolongo, F., Cariboni, J., Gatelli, D., Saisana, M. and S. Tarantola. 2008: Global sensitivity analysis: the primer. 
Wiley. com.

Schaller, R. R. 1997. Moore's law: past, present and future. Spectrum, IEEE 34:52-59.

Scheffer, M., A. A. Achterberg, and B. Beltman. 1984. Distribution of macroinvertebrates in a ditch in relation to the vegetation. Freshwater Biology 14:367-370.

Scheffer, M., S. H. Hosper, M. L. Meijer, B. Moss, and E. Jeppesen. 1993. Alternative equilibria in shallow lakes. Trends in Ecology \& Evolution 8:275-279.

Scheffer, M., and J. Beets. 1994. Ecological models and the pitfalls of causality. Hydrobiologia 275-276:115-124.

Scheffer, M., M. van den Berg, A. Breukelaar, C. Breukers, H. Coops, R. Doef, and M.-L. Meijer. 1994. Vegetated areas with clear water in turbid shallow lakes. Aquatic Botany 49:193-196.

Scheffer, M. 1998. Ecology of Shallow Lakes, Springer.

Scheffer, M., S. Carpenter, J. A. Foley, C. Folke, and B. Walker. 2001. Catastrophic shifts in ecosystems. Nature 413:591-596.

Scheffer, M., and S. R. Carpenter. 2003. Catastrophic regime shifts in ecosystems: linking theory to observation. Trends in Ecology \& Evolution 18:648656.

Scheffer, M., S. Szabó, A. Gragnani, E. H. van Nes, S. Rinaldi, N. Kautsky, J. Norberg et al. 2003. Floating plant dominance as a stable state. Proceedings of the National Academy of Sciences 100:4040-4045.

Scheffer, M., and E. H. van Nes. 2007. Shallow lakes theory revisited: various alternative regimes driven by climate, nutrients, depth and lake size. In: Shallow Lakes in a Changing World, Pages 455-466 in R. D. Gulati, E. Lammens, N. Pauw, and E. Donk, eds. Developments in Hydrobiology, Springer Netherlands.

Schindler, D.W. 1977. Evolution of phosphorus limitation in lakes. Science 195:260-262.

Schindler, D. W., R. Hecky, D. Findlay, M. Stainton, B. Parker, M. Paterson, K. Beaty et al. 2008. Eutrophication of lakes cannot be controlled by reducing nitrogen input: results of a 37-year whole-ecosystem experiment. Proceedings of the National Academy of Sciences 105:11254-11258.

Schindler, D. W., and R. E. Hecky. 2009. Eutrophication: More Nitrogen Data Needed. Science 324:721-722.

Schippers, P., H. Van de Weerd, J. De Klein, B. De Jong, and M. Scheffer. 2006. Impacts of agricultural phosphorus use in catchments on shallow lake 
water quality: About buffers, time delays and equilibria. Science of the Total Environment 369:280-294.

Schothorst, C. 1977. Subsidence of low moor peat soils in the western Netherlands. Geoderma 17:265-291.

Schultz, E. 1992. Waterbeheersing van de Nederlandse droogmakerijen, TU Delft, Delft University of Technology.

Scott, J. T., and M. J. McCarthy. 2010. Nitrogen fixation may not balance the nitrogen pool in lakes over timescales relevant to eutrophication management. Limnology and Oceanography 55:1265-1270.

Simons, T. J., and D. C. L. Lam. 1980. Some limitations of water quality models for large lakes: A case study of Lake Ontario. Water Resour. Res. 16:105-116.

Smith, V. H., G. D. Tilman, and J. C. Nekola. 1999. Eutrophication: impacts of excess nutrient inputs on freshwater, marine, and terrestrial ecosystems. Environmental Pollution 100:179-196.

Smith, S. D. P. 2014. The roles of nitrogen and phosphorus in regulating the dominance of floating and submerged aquatic plants in a field mesocosm experiment. Aquatic Botany 112:1-9.

Soetaert, K. and T. Petzoldt. 2010. Inverse modelling, sensitivity and Monte Carlo analysis in R using package FME. Journal of Statistical Software 33: 1-28.

Soetaert, K., T. Petzoldt, and R. W. Setzer. 2010. Solving differential equations in R: Package deSolve. Journal of Statistical Software 33:1-25.

Sollie, S., Janse, J. H., Mooij, W. M., Coops, H. and J.T. Verhoeven. 2008: The contribution of marsh zones to water quality in Dutch shallow lakes: A modeling study. Environmental management 42: 1002-1016.

Sondergaard, M., E. Jeppesen, T. L. Lauridsen, C. Skov, E. H. Van Nes, R. Roijackers, E. Lammens et al. 2007. Lake restoration: successes, failures and long-term effects. Journal of Applied Ecology 44:1095-1105.

Soranno, P. A., K. E. Webster, J. L. Riera, T. K. Kratz, J. S. Baron, P. A. Bukaveckas, G. W. Kling et al. 1999. Spatial Variation among Lakes within Landscapes: Ecological Organization along Lake Chains. Ecosystems 2:395-410.

Soranno, P. A., K. S. Cheruvelil, K. E. Webster, M. T. Bremigan, T. Wagner, and C. A. Stow. 2010. Using landscape limnology to classify freshwater ecosystems for multi-ecosystem management and conservation. BioScience 60:440-454.

Spaans, W., Booij, N., Praagman, N., Norman, R. and J. Lander. 1989. DUFLOW: A Micro-Computer Package for the Simulation of One-Dimensional Un- 
steady Flow in Open Channel Systems. Bureau SAMWAT, The Hague, Netherlands.

Steffen, W., K. Richardson, J. Rockström, S. E. Cornell, I. Fetzer, E. M. Bennett, R. Biggs et al. 2015. Planetary boundaries: Guiding human development on a changing planet. Science 347 .

Stowa \& MX.Systems. 2004. Duflow Modelling Studio: Duflow Manual version 3.7. STOWA.

Szabo, S., M. Scheffer, R. Roijackers, B. Waluto, M. Braun, P. T. Nagy, G. Borics et al. 2010. Strong growth limitation of a floating plant (Lemna gibba) by the submerged macrophyte (Elodea nuttallii) under laboratory conditions. Freshwater Biology 55:681-690.

Tilman, D. 1980. Resources: A Graphical-Mechanistic Approach to Competition and Predation. The American Naturalist 116:362-393.

Tilman, D. 1982, Resource competition and community structure Princeton, NJ, Princeton Univ. Press.

Trolle, D., Hamilton, D. P., Hipsey, M. R., Bolding, K., Bruggeman, J., Mooij, W. M., Janse, J. H., Nielsen, A., Jeppesen, E. and J. A. Elliott. 2012. A community-based framework for aquatic ecosystem models. Hydrobiologia 683: 25-34.

Trolle, D., Elliot, J. A., Mooij, W. M., Janse, J. H., Bolding, K., Hamilton, D. P. and E. Jeppesen. 2014: Advancing projections of phytoplankton responses to climate change through ensemble modelling. Environmental Modelling and Software 61: 371-379.

Van de Leemput, I. A., E. H. van Nes, and M. Scheffer. 2015. Resilience of Alternative States in Spatially Extended Ecosystems. PloS one 10.

Van der Heide, T., R. M. M. Roijackers, E. H. van Nes, and E. T. H. M. Peeters. 2006. A simple equation for describing the temperature dependent growth of free-floating macrophytes. Aquatic Botany 84:171-175.

Van Geest, G., F. Roozen, H. Coops, R. Roijackers, A. Buijse, E. Peeters, and M. Scheffer. 2003. Vegetation abundance in lowland flood plan lakes determined by surface area, age and connectivity. Freshwater biology 48:440-454.

Van Gerven, L. P. A., J. J. M. de Klein, D. J. Gerla, B. W. Kooi, J. J. Kuiper, and W. M. Mooij. 2015a. Competition for light and nutrients in layered communities of aquatic plants. The American Naturalist 186:72-83.

Van Gerven, L. P. A., R. J. Brederveld, J. J. M. de Klein, D. L. DeAngelis, A. S. Downing, M. Faber, D. J. Gerla et al. 2015b. Advantages of concurrent use of multiple software frameworks in water quality modelling using 
a database approach. Fundamental and Applied Limnology/Archiv für Hydrobiologie 185:5-20.

Van Kraalingen, D. W. G., Rappoldt, C. and H. H. van Laar. 2003. The Fortran simulation translator, a simulation language. European Journal of Agronomy 18: 359-361.

Van Laarhoven, P. J. M. and E. H. L. Aarts. 1987. Simulated annealing. Springer.

Van Liere, L., Janse, J., Jeuken, M., Van Puijenbroek, P., Schoumans, O., Hendriks, R., Roelsma, J., Jonkers, D., 2002. Effect of nutrient loading on surface waters in polder Bergambacht, The Netherlands. IAHS-publ. 273.

Van Liere, L., J. H. Janse, and G. H. P. Arts. 2007. Setting critical nutrient values for ditches using the eutrophication model PCDitch. Aquatic Ecology 41:443-449.

Van Nes, E. H. 2002. Controlling complexity in individual-based models of aquatic vegetation and fish communities, Wageningen University, Wageningen.

Van Nes, E. H., Lammens, E. H. and M. Scheffer. 2002. PISCATOR, an individualbased model to analyze the dynamics of lake fish communities. Ecological modelling 152: 261-278.

Van Nes, E. H. and M. Scheffer. 2003. Alternative attractors may boost uncertainty and sensitivity in ecological models. Ecological Modelling 159: 117-124.

Van Nes, E. H., and M. Scheffer. 2005. Implications of Spatial Heterogeneity for Catastrophic Regime Shifts in Ecosystems. Ecology 86:1797-1807.

Van Oevelen, D., Van den Meersche, K., Meysman, F. J., Soetaert, K., Middelburg, J. J. and A. F. VÃl'zina. 2010. Quantifying food web flows using linear inverse models. Ecosystems 13: 32-45.

Van Puijenbroek, P. J. T. M., Janse, J. H. and J. M. Knoop. 2004. Integrated modelling for nutrient loading and ecology of lakes in The Netherlands. Ecological Modelling 174: 127-141.

Van Rossum, G. and F. L. Drake. 2001. Python Reference Manual, PythonLabs, Virginia, USA.

Verdonschot, R. C., H. E. Keizer-Vlek, and P. F. M. Verdonschot. 2012. Development of a multimetric index based on macroinvertebrates for drainage ditch networks in agricultural areas. Ecological indicators 13:232-242.

Verdonschot, R. C. M., and Verdonschot, P. F. M. 2014. Shading effects of freefloating plants on drainage-ditch invertebrates. Limnology 15:225-235.

Volterra, V. 1928. Variations and fluctuations of the number of individuals in 
animal species living together. J. Cons. Int. Explor. Mer 3:3-51.

Wagner, G. M. 1997. Azolla: A review of its biology and utilization. Botanical Review 63:1-26.

Watanabe, I. 1982. Azolla-Anabaena symbiosis-its physiology and use in tropical agriculture, Pages 169-185 Microbiology of tropical soils and plant productivity, Springer.

Weissing, F. J., and J. Huisman. 1994. Growth and competition in a light gradient. Journal of Theoretical Biology 168:323-336.

Williamson, M. H. 1957. An Elementary Theory of Interspecific Competition. Nature 180:422-425.

Witteveen + Bos. 2008a. Watersysteemanalyse Loosdrechtse Plassen. Report nr. LOOS20-1-1 (In Dutch).

Witteveen + Bos. 2008b. Nadere uitwerking waterkwaliteit en ecologische kwaliteit Wieringerrandmeer. Report nr. WRW5-32 (In Dutch).

Witteveen + Bos. 2009. Effecten peilbeheer en waterberging Oldambtmeer. Report nr. VDM53-1-1 (In Dutch).

Witteveen + Bos. 2010a. Ecologische modellering Loenderveense Plas en Terra Nova. Report nr. LN4-1-1 (In Dutch).

Witteveen + Bos. 2010b. Waterkwaliteit herinrichting Nieuwe Driemanspolder. Report nr. ZTM99-1-1 (In Dutch).

Witteveen + Bos. 2013a. Toetsing, verbetering en ontsluiting ecologische modellen PCLake en PCDitch aan de hand van praktijktoepassingen: case Zuidlaardermeer. Report nr. STO170-1-10 (In Dutch).

Witteveen + Bos. 2013b. Toetsing, verbetering en ontsluiting ecologische modellen PCLake en PCDitch aan de hand van praktijktoepassingen: case Bergse Plassen. Report nr. STO170-1-13 (In Dutch).

Witteveen + Bos. 2013c. Toetsing, verbetering en ontsluiting ecologische modellen PCLake en PCDitch aan de hand van praktijktoepassingen: case Stad van de Zon. Report nr. STO170-1-14 (In Dutch).

Wolf, J., M. Hack-ten Broeke, and R. Rötter. 2005. Simulation of nitrogen leaching in sandy soils in The Netherlands with the ANIMO model and the integrated modelling system STONE. Agriculture, ecosystems \& environment 105:523-540.

Wolfram, S. 1999. The MATHEMATICA Book, Version 4. Cambridge university press.

Yoshiyama, K., Jarad P. Mellard, E. Litchman, and Christopher A. Klausmeier. 2009. Phytoplankton Competition for Nutrients and Light in a Stratified Water Column. The American Naturalist 174:190-203. 
Zevenboom, W., J. Van Der Does, K. Bruning, and L. R. Mur. 1981. A nonheterocystous mutant of Aphanizomenon flos-aquae, selected by competition in light-limited continuous culture. FEMS Microbiology Letters 10:11-16. 


\section{Summary}

The Netherlands is well-known for its extended networks of drainage ditches, with a total ditch length of about $300.000 \mathrm{~km}$. Their main function is to enable agriculture by draining water. Nonetheless, ditches also have important ecological functions. They serve as ecological corridors and harbor a high biodiversity in which water plants play a crucial role. The last decades, the ecological quality of ditches is at stake. Enhanced nutrient inputs promoted the invasion by dense mats of free-floating plants like duckweed. Underneath these mats the water becomes dark and anoxic, which severely constrains aquatic life.

In this thesis I developed new concepts to better understand, predict and combat the dominance by free-floating plants in ditches. The following questions are addressed. Are floating plants a self-stabilizing state - an alternative stable state - which would make it more difficult to combat floating-plant dominance (chapter 2)? Does it make sense to fight floating-plant dominance by reducing nitrogen $(\mathrm{N})$ inputs to the ditches or will it lead to an invasion of floating plants that can fix $\mathrm{N}_{2}$ from the atmosphere (chapter 3)? What about spatial aspects, does the vulnerability of a ditch to floating plants depend on the position of a ditch in a polder, like its distance to the polder outlet (chapter 4)? To answer these questions, I used ecological models that predict the abundance of free-floating plants based on the competition for nutrients and light with other plants such as submerged plants, and where possible validated these models with field data. Starting from the ecosystem model PCDitch, I developed and combined models with different complexity to see how theoretical concepts, developed in minimal models, translate to the ecosystem level. Chapter 5 deals with a method that facilitates this up- and downscaling in model complexity.

Are floating plants an alternative stable state? To answer this question I extended mechanistic resource competition theory with a framework (minimal model) describing the competition of floating and submerged plants for 
light and nutrients. The model predicts that the competitive advantage of floating plants - they have a primacy for light and shade submerged plants, giving rise to asymmetry in competition for light - makes that floating plants always dominate at high supply of light and nutrients. At intermediate nutrient supply, there can be alternative stable states: either the submerged plants or the floating plants dominate depending on who established first. However, based on the traits of common floating plants (duckweed; Lemna) and submerged plants (waterweed; Elodea) the model predicts, in line with field data, that floating plants are not an alternative stable state. Furthermore, from a theoretical point of view this study shows that the asymmetry in light competition ensures that common rules from standard competition theory do not apply anymore. Like the $\mathrm{R}^{*}$ rule, which states that the species that can persist at the lowest resource levels always wins the competition.

Can duckweed-dominance be combatted by reducing $\mathrm{N}$ inputs to the ditches? Or does this promote other floating plants like water fern (Azolla) that can fix $\mathrm{N}_{2}$ from the atmosphere? Important is the question whether such $\mathrm{N}_{2}$-fixers can provide enough $\mathrm{N}$ to prevent $\mathrm{N}$-limitation and keep the system P-limited, which would make steering on $\mathrm{N}$ inputs ineffective. To investigate this, I considered the competition between Lemna and Azolla for N, P and light. Both a minimal model, an ecosystem model (PCDitch) and field data reveal that $\mathrm{N}_{2}$-fixation is unlikely to lead to P-limitation. This can be explained by $\mathrm{N}_{2}$-fixers typically requiring higher $\mathrm{P}$ concentrations to persist, implying that they cannot keep the P concentration low enough for non- $\mathrm{N}_{2}$-fixers to become P-limited. In combination with field data that hint at constraints on $\mathrm{N}_{2}$-fixation that prevent $\mathrm{N}_{2}$-fixers from becoming abundant at low $\mathrm{N}$ availability, this suggests that it certainly pays off to combat floating plant-dominance by reducing $\mathrm{N}$ inputs.

Is every ditch in a polder equally vulnerable to floating plants? Each ditch in a polder receives water and nutrients from the adjacent land. This leads to a spatial gradient in water flow and associated nutrient loading, from low in the remote polder sites to high in the direction of the polder outlet where the water leaves the polder. I explored if this spatial gradient affects the vulnerability of a ditch to floating plants, by investigating with a simple nutrient model how this gradient affects the nutrient concentration of the ditches and by subsequently predicting the gradient's effect on the ditch ecology by applying the ecosystem model PCDitch spatially, through coupling PCDitch to the 1-D hydrodynamic model SOBEK. Surprisingly, we found that every ditch is equally vulnerable to floating plants, despite the spatial gradient in water flow and nutrient loading. 
It turned out that the ecological state of each ditch could already be predicted by regarding only the lateral supply of water and nutrients from the adjacent land, and not the supply from upstream ditches. However, these findings are violated when there is spatial heterogeneity in the water and nutrient supply from the adjacent land or in ditch characteristics like depth and sediment type. Then, the chance on floating-plant dominance differs throughout the network and a spatial modelling approach (PCDitch-SOBEK) is required to predict this chance.

Developing and combining models of different complexity plays an important role in this thesis. To do so, I used a Database Approach To Modelling (DATM), a recently developed method in which a model is stored in tables in a clear and clean way, which facilitates model development. In addition, with DATM a model can be automatically implemented in a modelling environment of choice. This relieves technical implementation issues and leaves room to focus on ecology rather than technology. I illustrated the use of DATM by implementing and analyzing the ecosystem model PCDitch and its twin model for shallow lakes PCLake in different modelling environments by using DATM. This showed that DATM allows one to use the environment one is familiar with and eases the switch to other environments for complementary analyses, including analysis in a spatial 1-D to 3-D setting.

The insights provided by this thesis can help us to improve the ecological quality of ditches. A challenging task, given the fast human-driven environmental changes at both local and global level. To predict and to anticipate the effect of these changes on the ecology, it is essential to understand how the ditch ecosystem functions. The developed and applied methods described in this thesis may be helpful in that. For example, using models of different complexity makes it possible to translate fundamental theory to the ecosystem scale, which is essential to better grasp the behavior of an ecosystem. Furthermore, the in this thesis established coupling between PCDitch and SOBEK breaks new grounds for spatial ecosystem modelling. In combination with the growing amount of remote sensing data from satellites and drones, which allow for the continuous and potentially real-time validation and calibration of spatial ecosystem models, such a spatial approach has the potential to greatly increase our ecological understanding of ditches. These advances facilitate the development of successful management strategies that make our ditch ecosystems future-proof. 



\section{Samenvatting}

Nederland staat bekend om zijn uitgebreid netwerk aan sloten, met een totale slootlengte van wel $300.000 \mathrm{~km}$. Hun voornaamste functie is het afvoeren van water om landbouw mogelijk te maken. Sloten hebben echter ook een belangrijke ecologische functie. Ze dienen als ecologische connectors en herbergen een grote biodiversiteit waarbij waterplanten een cruciale rol spelen. De ecologische kwaliteit van sloten staat echter al tientallen jaren onder druk. De verhoogde toevoer van nutriënten heeft gezorgd voor de opkomst van dikke lagen aan drijvende planten zoals kroos. Onder zo'n laag wordt het water donker en zuurstofarm, met negatieve gevolgen voor het leven in de sloot.

In dit proefschrift heb ik nieuwe concepten ontwikkeld om de dominantie van drijvende planten beter te kunnen begrijpen, voorspellen en tegen te gaan. Ik ga in op de volgende vragen. Houden drijvende planten zichzelf in stand - oftewel zijn ze een alternatieve stabiele toestand - waardoor ze zich moeilijk laten bestrijden (hoofdstuk 2)? Heeft het nut om drijvende planten te bestrijden door de belasting met stikstof $(\mathrm{N})$ te verlagen of leidt dit tot de opkomst van drijvende planten die $\mathrm{N}_{2}$ uit de lucht kunnen opnemen (hoofdstuk 3)? Hoe zit het ruimtelijk, hangt de kans op drijvende planten af van de ligging van de sloot, zoals de afstand tot het poldergemaal (hoofdstuk 4)? Om deze vragen te beantwoorden gebruik ik modellen die het voorkomen van drijvende waterplanten voorspellen op basis van hun competitie om licht en nutriënten met andere waterplanten zoals ondergedoken planten en valideer ik de modellen waar mogelijk met veldgegevens. Met het ecosysteem model PCDitch als startpunt heb ik modellen met verschillende complexiteit ontwikkeld om zo theoretische concepten, gevat in mini-modellen, door te vertalen naar ecosysteemniveau. Hoofdstuk 5 gaat in op een methode die modelontwikkeling en het op- en neerschalen in modelcomplexiteit faciliteert.

Zijn drijvende planten een alternatieve stabiele toestand? Om deze vraag 
te beantwoorden heb ik competitietheorie uitgebreid met een raamwerk (mini-model) dat de competitie beschrijft van drijvende en ondergedoken planten om licht en nutriënten. Het mini-model voorspelt dat het competitieve voordeel van drijvende planten - ze krijgen als eerste het licht en beschaduwen de ondergedoken planten, leidend tot asymmetry in competitie voor licht - ervoor zorgt dat drijvende planten altijd domineren bij hoge aanvoer van licht en nutriënten. Wanneer nutriënten limiterend zijn, kan er sprake zijn van alternatieve stabiele toestanden: zowel de ondergedoken als de drijvende planten kunnen domineren afhankelijk van welke plant zich het eerste vestigde. Echter, op basis van de eigenschappen van veel voorkomende drijvers (kroos; Lemna) en ondergedoken planten (waterpest; Elodea) voorspelt het model, in overeenstemming met veldwaarnemeningen, dat drijvende planten geen alternatieve stabiele toestand zijn. Vanuit theoretisch oogpunt laat deze studie zien dat de asymmetry in lichtcompetitie ervoor zorgt dat algemene regels uit standaard theorie over (symmetrische) competitie niet meer opgaan. Zoals de $\mathrm{R}^{*}$ regel die stelt dat een soort de competitie wint als hij kan bestaan bij de laagste niveaus aan resources.

Kun je drijvende planten tegengaan door de $\mathrm{N}$ belasting terug te brengen? Of speelt het planten in de kaart die $\mathrm{N}_{2}$ kunnen fixeren uit de lucht en zo uiteindelijk voldoende $\mathrm{N}$ aan het systeem toevoegen zodat niet $\mathrm{N}$ maar fosfor (P) limiterend is voor de groei van drijvers, waardoor sturen op $\mathrm{N}$ geen zin heeft. Om dit te onderzoeken heb ik de competitie beschouwd tussen drijvers die wel (Azolla) en geen (Lemna) $\mathrm{N}_{2}$ kunnen fixeren. Zowel een mini-model, een ecosysteemmodel en veldgegevens laten zien dat $\mathrm{N}_{2}$-fixatie niet altijd leidt tot P-limitatie. Rede hiervoor is dat $\mathrm{N}_{2}$-fixers in het algemeen hogere $\mathrm{P}$ concentraties nodig hebben om te kunnen bestaan en daardoor de $\mathrm{P}$ concentratie in het water niet laag genoeg kunnen houden om non- $\mathrm{N}_{2}$-fixers P-gelimiteerd te laten zijn. Dit suggereert dat het tegengaan van drijvende planten door het verlagen van de $\mathrm{N}$ belasting wel degelijk zin heeft, ook omdat veldgegevens wijzen op belemmeringen van de $\mathrm{N}_{2}$-fixatie die de opkomst van $\mathrm{N}_{2}$-fixers bij lage $\mathrm{N}$ belasting verhinderen.

Is elke sloot in een polder even kwetsbaar voor drijvende planten? Iedere poldersloot ontvangt water en nutriënten van het aangrenzende perceel. Dit leidt tot een ruimtelijke gradiënt in waterstroming en nutriëntenbelasting: van klein in de afgelegen polderdelen tot groot in de richting van het poldergemaal. Ik heb onderzocht of deze gradiënt invloed heeft op de kwetsbaarheid van sloten voor drijvende planten, door de toestand van elke sloot in het netwerk te voorspellen met een mini-model en een ecosysteemmodel 
(PCDitch) gekoppeld aan een 1-D hydrodynamisch model (SOBEK). Verrassend genoeg voorspellen beide modellen dat iedere sloot even kwetsbaar is voor drijvende planten, ongeacht de ruimtelijke gradiënt in waterstroming (verblijftijden) en nutriëntenbelasting. Het bleek dat je de ecologische toestand van de sloot al kan voorspellen wanneer je alleen kijkt naar de lokale aanvoer van water en nutriënten vanuit het aangrenzende perceel, en niet de aanvoer vanuit bovenstroomse sloten. Deze bevindingen gaan echter niet meer op wanneer er ruimtelijke verschillen zijn in de water- en nutriëntenaanvoer vanuit de percelen of in slootkenmerken zoals diepte en sedimenttype. Dan heeft elke sloot namenlijk een andere kans op kroos en heb je een ruimtelijk model nodig zoals PCDitch-SOBEK om deze kans te voorspellen.

Het ontwikkelen en combineren van modellen met verschillende complexiteit speelt een belangrijke rol in dit proefschrift. Daarvoor heb ik gebruik gemaakt van een Database Approach To Modelling (DATM), een recent ontwikkelde methode waarin je modellen op een overzichtelijke manier in tabelvorm opslaat en zo modelonwikkeling faciliteert. Daarnaast kun je een model vanuit DATM automatisch implementeren in een modelomgeving naar keuze. Dit ontneemt technische zorgen over de implementatie en biedt meer ruimte je te richten op ecologie in plaats van technologie. Ik ben ingegaan op het gebruik van DATM door het ecosysteemmodel PCDitch en het tweelingmodel voor ondiepe meren PCLake vanuit DATM te implementeren en analyseren in verschillende modelomgevingen. Dit laat zien dat DATM je vrij laat de modelomgeving te gebruiken waarmee je het meest bekend bent, en de switch faciliteert naar andere omgevingen voor het doen van aanvullende analyses, inclusief analyses in een ruimtelijke 1-D tot 3-D setting.

De in dit proefschrift opgedane inzichten kunnen ons helpen om de ecologische kwaliteit van sloten te verbeteren. Een uitdagende taak, zeker gezien de grote veranderingen in het milieu die zich wereldwijd voltrekken. Om te kunnen anticiperen op deze toekomstige veranderingen is het van groot belang het ecologisch functioneren van een sloot goed te doorgronden. De in dit proefschrift aangedragen technieken en methodes kunnen je daarbij helpen. Het gebruik van modellen van verschillende complexiteit maakt het bijvoorbeeld mogelijk om fundamentele kennis te vertalen naar ecosysteemniveau. Een essentiële stap om het ecosysteemgedrag beter te snappen. Verder biedt de in dit proefschrift tot stand gebrachte koppeling tussen PCDitch en SOBEK veel mogelijkheden voor het ruimtelijk modelleren van sloten. In combinatie met de groeiende hoeveelheid data van satellieten en drones, die je kan inzetten om de ruimtelijke modelleren voortdurend en mogelijk zelfs real- 
time te valideren en kalibreren, zal dit leiden tot een sterk verbeterd inzicht in het functioneren van sloten. Al deze ontwikkelingen helpen ons om succesvolle beheersstrategieën te definiëren die onze sloten toekomstbestendig maken. 


\section{Dankwoord}

Zowel na mijn studie geofysica als hydrologie werd me de mogelijk geboden een promotieonderzoek te doen. Daar zag ik destijds weinig heil in. Was het niet saai om 4 jaar lang onderzoek te doen aan eenzelfde onderwerp? Toen in 2012 wederom een promotieonderzoek op mijn pad kwam besloot ik, nadat ik 4 jaar lang aan de onderzoekssfeer had geproefd bij Alterra, de uitdaging alsnog aan te gaan. Driemaal scheepsrecht. Ik ben blij dat ik deze keuze heb gemaakt. Ik kijk terug op een zeer leuke en leerzame tijd. En nee, het was bij lange na niet saai.

Promoveren doe je niet alleen. Ik wil dan ook graag de mensen om me heen bedanken die me hebben geholpen het promotietraject te volbrengen. Allereerst mijn promotor Wolf Mooij. Bedankt voor je inhoudelijke bijdrage, de fijne gesprekken, de mentale steun en ons gedeelde gevoel voor humor! Mijn co-promotor Jeroen de Klein. Jij attendeerde me destijds op dit onderzoek en gezien onze eerdere fijne samenwerking, op zowel inhoudelijk als persoonlijk vlak, wist ik dat dit onderzoek met jou als dagelijks begeleider in goede handen was. Bedankt voor de fijne begeleiding! Mijn co-promotor Jan Janse. Zonder jou, als ontwikkelaar van de modellen PCLake en PCDitch, had dit onderzoeksproject nooit bestaan. Bedankt voor je kalmte, humor en deskundige hulp. Bas van der Wal en STOWA, voor het erkennen van het belang van dit onderzoek en de financiering ervan. De overige leden van het PCLake-PCDitch-projectteam, Noemi von Meijenfeldt, Sebastiaan Schep en

Bob Brederveld. Bedankt voor de gezelligheid en de leuke en nuttige projectoverleggen, met als persoonlijk hoogtepuntje het 'nerd-overleg'. En niet te vergeten mijn collega-aio's en PC-partners in crime, Annette Janssen en Jan Kuiper. Annette, mijn rots in de branding, bij jou kon ik altijd terecht voor inhoudelijke of praktische vragen, of voor een gezellig potje ping-pong! Jan, mijn kamergenoot. Bedankt voor de lol die we samen hebben gehad en voor onze complementariteit. Ik hielp jou met technische zaken en jij hiep mij met het fijnslijpen van de boodschap en tekst van artikelen. Een super team! 
Ik had het voorrecht te mogen werken in een fijne werkomgeving. Ik voelde me als een vis in het water bij het Nederlands Instituut voor Ecologie waar ik m'n onderzoek heb uitgevoerd. De sfeer was stimulerend en gezellig. De seminars, de borrels, het kerstdiner, het kerstkoor, de NIOO-dagen, 'the Chinese food', 'the birthday parties' en de PhD-uitjes zullen me nog lang heugen. Hoogtepunt was het Shallow Lakes symposium in Antalya waar vrijwel ons hele departement aquatische ecologie was vertegenwoordigd en waar we met een aantal collega's nog een kleine vakantie aan hebben vastgeplakt. Echt een super tijd; toch best prima zo'n all-inclusive resort.

Ik wil m'n NIOO-collega's dan ook graag bedanken voor de leuke gesprekken en momenten die we samen hebben beleefd. Allereerst de staf, postdocs en assistenten van mijn departement (Ellen, Lisette, Liesbeth, Steven, Dedmer, Joost, Tania, Casper, Jing, Alena, Michaela, Suzanne en de mannen met de 'minion-pakken' Nico, Dennis en Erik), collega's van de buur-departementen (Maaike, Annelies, Lysanne, Erik, Thomas, Marleen, Rascha, Irene, Femke, Kristin, Roos, Annemieke, Anne, Paolo, Marta, Stijn, Jasper, Veronica, Lucia, Viola, en iedereen die ik nu nog vergeet, thanks!) and last but certainly not least de gezellige aio-groep van mijn departement; de Nederlandse afvaardiging (het Vierlingsbeektrio Michiel, Bart en Ralf; Laura, Sven, Thijs, Karen, Mandy, Annette en Jan) and of course the international $\mathrm{PhD}$ delegation adding color and delicious food to our department (Kim, Peiyu, Antonella, Libin and Wei).

Verder wil ik graag bedanken:

- De leerstoelgroep Aquatic Ecology and Water Quality Management van de Wageningen Universiteit (Marten, Bart, Jeroen, Egbert, Miquel, Edwin, John, Frits, Nancy, Arie, Marlies, Arie, Ingrid, Jelle, Bregje, Bastiaan, Jacqueline, etc.), voor de enerverende teamuitjes, inhoudelijke discussies, leuke gesprekken en lunchlezingen. Ik voelde me altijd welkom aan 'de andere kant van de straat'!

- Een inspirerende groep aan co-auteurs; Bob Kooi, Michel Jeuken, Daan Gerla, Hans Paerl en niet te vergeten de leden van het internationale Aquatic Ecosystem MOdelling Network (AEMON). Thanks for the fruitful cooperation!

- De foodweb discussion group (Jeroen, Cassandra, Sanne, Amber, Jelle, etc.), voor de leuke discussies en borrels na afloop :-)

- Mijn voormalige Alterra collega's (Frank, Piet, Oscar, Dorothee, Leo, Erwin, Dennis, Joop K, Joop H., Rob K., Martin, Jan, Franciska, Peter, Tim, 
Cees, Erik, Christian, Harry, Paul, Ab, etc.), voor mijn fijne en leerzame tijd bij Alterra, met een speciaal bedankje voor mijn 'mentoren' Robert en Rob H.

- Mijn squashmaatjes van de WUR (Ton, Martin, Peter en Lawrence), voor de wekelijkse squashsessies. Ik daag jullie in de toekomst graag nog een keer uit!

- Mijn vriendengroep (Ronny, Roy, Roel, Rob, Eddy, Maurice, Marten, Xander, Joost en Gijs), voor bruiloften, partijen, baby-showers, maar vooral het jaarlijkse mannenweekend!

- Mijn pubquizmaatjes (Michel, Simcha, Dirk en Patrick), voor de nodige ontspanning op de woensdagavond :-)

- Mijn bandgenootjes van Cell29, Valentine en Ex Libris, voor veel lol en muzikaal vertier!

- Mijn nieuwe collega's bij RoyalHaskoningDHV, voor het warme welkom!

En dan natuurlijk mijn familie niet te vergeten! De mensen die altijd voor me klaar staan. Peter-Jan en Marijke, Dana, Mark en Chris, ik kijk alweer uit naar het volgende zaterdagmiddag-biertje. Pap en mam, Inge, Michel, Tijn en Pleun, fijn dat ik altijd op jullie terug kan vallen. En Michel, super bedankt voor het ontwerpen van de cover. En als laatste de belangrijkste personen en steun en toeverlaat in mijn leven, Kelli, Tibbe en Gus. Jullie zijn mijn alles! 



\section{About the author}

Luuk van Gerven was born on 3 September 1980 in Tegelen. He grew up in Swalmen, a village in the south of the Netherlands, where as a child he already became fascinated by the beauty of the forest and the meandering stream the Swalm. After graduating from Atheneum at Bisschoppelijk College Broekhin Roermond in 1998, he moved to Utrecht to study Geophysics at the Utrecht University. He specialized in seismology and tectonophysics, and investigated the composition of Australia's deep subsurface to obtain his master's degree in 2003. From 2004 to 2007, Luuk followed a second master's program in Hydrology and Water Quality at the Wageningen University, where he graduated on the flood risk of the river Meuse and on the meandering patterns of the Mahakam River (Kalimantan, Indonesia). Besides being a dedicated student, Luuk also was and still is a passionate musician. He played the bass guitar in After Forever (1996-2007). With this band he released five albums and performed at many venues and festivals across Europe, North America and South America. In 2008, Luuk started his professional career as a hydrologist at Alterra, where he focused on measuring and modelling the pathways of water and nutrients in surface waters. In 2012, Luuk was appointed as a PhD candidate at the Netherlands Institute of Ecology (NIOOKNAW). He studied, in collaboration with the Wageningen University, how the pathways of water and nutrients affect the ecology of surface waters, with a special focus on aquatic plants in ditches. His work resulted in this thesis. From July 2016 onwards, Luuk is employed at RoyalHaskoningDHV as an advisor on the ecological and chemical quality of surface waters. 



\section{Publications}

\section{Peer reviewed articles}

van Gerven, L. P. A., J. J. M. de Klein, D. J. Gerla, B. W. Kooi, J. J. Kuiper, and W. M. Mooij. 2015. Competition for Light and Nutrients in Layered Communities of Aquatic Plants. The American Naturalist 186: 72-83.

van Gerven, L. P. A., J. J. Kuiper, J. H. Janse, A. B. G. Janssen, M. Jeuken, W. M. Mooij and J. J. M. de Klein, 2016. How regime shifts in connected aquatic ecosystems are affected by the typical downstream increase of water flow. Accepted for publication in Ecosystems.

van Gerven, L. P. A., R. Brederveld, J. de Klein, D. DeAngelis, A. Downing, M. Faber, D. Gerla, J. 't Hoen, J. H. Janse, A. B. G. Janssen, M. Jeuken, B. W. Kooi, J. J. Kuiper, B. Lischke, S. Liu, T. Petzoldt, S. A. Schep, S. Teurlincx, C. Thiange, D. Trolle, E. H. van Nes, and W. M. Mooij, 2015. Advantages of concurrent use of multiple software frameworks in water quality modelling using a database approach. Fundamental and Applied Limnology 185: 5-20.

van Gerven, L. P. A. , F. Deschamps, and R. D. van der Hilst. 2004. Geophysical evidence for chemical variations in the Australian Continental Mantle. Geophysical Research Letters 31.

Hoitink, A., A. Dommerholt, and L. P. A. van Gerven. 2008. Hydraulic design of a tilting weir allowing for periodic fish migration. Journal of Hydraulic Engineering 134: 1559-1569.

Hu, F, K. Bolding, J. Bruggeman, E. Jeppesen, M. R. Flindt, L. P. A. van Gerven, J. H. Janse, A. B. G. Janssen, J. J. Kuiper, W. M. Mooij, and D. Trolle, 2016. FABM-1 PCLake-linking aquatic ecology with 2 hydrodynamics. Geoscientific Model Development 9(2).

Janssen, A. G., G. Arhonditsis, A. Beusen, K. Bolding, L. Bruce, J. Bruggeman, R.M. Couture, A. S. Downing, J. A. Elliott, M. A. Frassl, G. Gal, D. J. Gerla, M. R. Hipsey, F. Hu, S. C. Ives, J. H. Janse, E. Jeppesen, K. D. Jöhnk, D. Kneis, X. 
Kong, J. J. Kuiper, M. K. Lehmann, C. Lemmen, D. Özkundakci, T. Petzoldt, K. Rinke, B. J. Robson, R. Sachse, S. A. Schep, M. Schmid, H. Scholten, S. Teurlincx, D. Trolle, T. A. Troost, A. A. Van Dam, L. P. A. Van Gerven, M. Weijerman, S. A. Wells, W. M. Mooij, 2015. Exploring, exploiting and evolving diversity of aquatic ecosystem models: a community perspective. Aquatic Ecology 49: 513-548.

Kong, X., Q. He, B. Yang, W. He, F. Xu, A. B. G. Janssen, J. J. Kuiper, L. P. A. van Gerven, N. Qin, Y. Jiang, and W. Liu, 2016. Hydrological regulation drives regime shifts: evidence from paleolimnology and ecosystem modeling of a large shallow Chinese lake. Global Change Biology.

Kuiper, J. J., C. van Altena, P. C. de Ruiter, L. P. van Gerven, J. H. Janse, and W. M. Mooij. 2015. Food-web stability signals critical transitions in temperate shallow lakes. Nature communications 6.

Mooij, W. M., R. J. Brederveld, J. J. M. de Klein, D. L. DeAngelis, A. S. Downing, M. Faber, D. J. Gerla, M. R. Hipsey, J. ’t Hoen, J. H. Janse, A. B. G. Janssen, M. Jeuken, B. W. Kooi, B. Lischke, T. Petzoldt, L. Postma, S. A. Schep, H. Scholten, S. Teurlincx, C. Thiange, D. Trolle, A. A. van Dam, L. P. A. van Gerven, E. H. van Nes, and J. J. Kuiper, 2014. Serving many at once: How a database approach can create unity in dynamical ecosystem modelling. Environmental Modelling \& Software 61: 266-273.

\section{Reports}

van Gerven, L.P.A., B. van der Grift, R.F.A. Hendriks, H.M. Mulder en T.P. van Tol-Leenders, 2011. Nutriëntenhuishouding in de bodem en het oppervlaktewater van de Krimpenerwaard. Bronnen, routes en sturingsmogelijkheden. Alterra Rapport 2220, Wageningen.

van Gerven, L.P.A., R.F.A. Hendriks, J. Harmsen, V. Beumer, P. Bogaart, 2011. Nalevering van fosfor naar het oppervlaktewater vanuit de waterbodem in een veengebied. Metingen in de Krimpenerwaard. Alterra rapport 2217, Wageningen.

van Gerven, L.P.A., J.J.M. de Klein, F.J.E. van der Bolt, 2010. Retentie van nutriënten in het oppervlaktewater; Meetcampagne in het Zeegserloopje. Alterra Rapport 2133, Wageningen.

van Gerven, L.P.A., A.A.M.F.R. Smit, P. Groenendijk, FJ.E. van der Bolt, J.J.M. de Klein, 2009. Retentieschatting van $\mathrm{N}$ en $\mathrm{P}$ in het oppervlaktewater op verschillende schaalniveaus. Alterra Rapport 1848, Wageningen.

van Gerven, L.P.A., H.M. Mulder, C. Siderius, T.P. van Tol-Leenders, A.A.M.F.R. 
Smit, 2009. Analyse van de invloed van processen op de nutriëntenconcentraties in het oppervlaktewater - Een modelstudie. Alterra Rapport 1855, Wageningen.

Dik, P.E., M.H.J.L. Jeuken, L.P.A. van Gerven, 2009. SWQN, Manual version 3.0. Alterra Rapport 1226.3, Wageningen.

Jeuken, M.H.J.L., L.P.A. van Gerven, 2008. NuswaLite; Manual version 3.0. Alterra Rapport 1226.4, Wageningen.

Mulder, H.M., L.P.A. van Gerven, E.P. Querner, A.K. van der Werf, 2009. Waterkwaliteit op het landgoed Het Lankheet; Zuiverende werking van rietvelden en de Buurserbeek. Alterra Rapport 1878, Wageningen.

Siderius, C., J. Roelsma, H.M. Mulder, L.P.A. van Gerven, R.F.A. Hendriks en T.P. van Tol-Leenders, 2011. Kalibratie Modelsysteem Monitoring Stroomgebieden. Alterra Rapport 2216, Wageningen.

Siderius C., P. Groenendijk, L.P.A. van Gerven, M.H.J.L. Jeuken, A.A.M.F.R Smit, 2008. Process description of NuswaLite; a simplified model for the fate of nutrients in surface waters. Alterra Rapport 1226.2, Wageningen.

Smit, A.A.M.F.R, C. Siderius, L.P.A. van Gerven, in press. Process description of SWQN; A simplified hydraulic model. Alterra Rapport 1226.1, Wageningen.

van Boekel, E.M.P.M., P. Bogaart, L.P.A. van Gerven, T. van Hattum, R.A.L. Kselik, H.T.L. Massop, H.M. Mulder, P.E.V. van Walsum en FJ.E. van der Bolt, 2013. Evaluatie landbouw en KRW: evaluatie meststoffenwet 2012: deelrapport ex post. Alterra Rapport 2326, Wageningen.

van Boekel, E.M.P.M., L.P.A. van Gerven, T. van Hattum, V.G.M. Linderhof, H.T.L. Massop, H.M. Mulder, N.B.P. Polman, L.V. Renaud en D.J.J. Walvoort, 2011. Ex-ante evaluatie landbouw en KRW: Bijdrage van het voorgenomen beleid en aanvullende (landbouwkundige) maatregelen op de realisatie van de KRW-nutriëntendoelstelling. Alterra Rapport 2121, Wageningen.

Veraart, J.A., L.P.A. van Gerven, 2013. Verzilting, klimaatverandering en de kaderrichtlijn Water. Casestudie het boezemstelsel van Schieland. Onderzoeksrapport KvK Thema 2 Zoetwatervoorziening en waterkwaliteit, Alterra, Wageningen.

Buck, A.J. de, L.P.A. van Gerven, J. van Kleef, J.R. van der Schoot, G.C.A. Wijk, A. Buijert, FJ.E. van der Bolt, 2012. Helofytenfilters in sloten: schoonheid door eenvoud. PPO rapport 517, WUR/PPO, Lelystad. 


\section{Other publications}

van Gerven, L.P.A., A.J.F Hoitink, in press. Analysis of River planform geometry with wavelets: Application to the Mahakam River reveals geographical zoning. Submitted to: 6th Symposium on River, Coastal and Estuarine Morphodynamics (RCEM), sept 2009, Sante Fe, Argentina.

Hendriks, C.M.A., J.W.R. Twisk, L.P.A. van Gerven, J. Harmsen, 2013. Sulfaat in veenweiden: gebiedsvreemd of gebiedseigen? $\mathrm{H} 2 \mathrm{O}$ : tijdschrift voor watervoorziening en afvalwaterbehandeling (mei). 


\section{PE\&RC Training and Education Statement}

With the training and education activities listed below the $\mathrm{PhD}$ candidate has complied with the requirements set by the C.T. de Wit Graduate School for Production Ecology and Resource Conservation (PE\&RC) which comprises of a minimum total of 32 ECTS ( $=22$ weeks of activities)

\section{Review of literature (6 ECTS)}

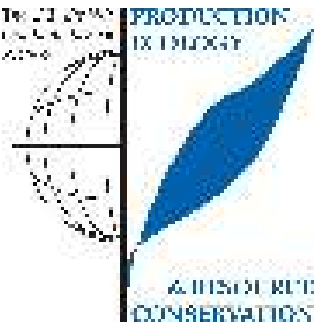

The ecology of linear waters in temperate climate regions: drivers and responses (2012)

\section{Writing of project proposal (4.5 ECTS)}

- Factors controlling the vegetation composition of linear waters (2012)

\section{Post-graduate courses (4.6 ECTS)}

Bayesian statistics; PE\&RC (2012)

- Modelling critical transitions in nature and society; SENSE (2014)

- Consumer-resource interactions; PE\&RC (2014)

\section{Laboratory training and working visits (1.2 ECTS)}

- Work visit on challenges and opportunities in ecosystem modelling with PCLake/PCDitch; oral presentation; Aarhus University, Denmark (2014)

Invited review of (unpublished) journal manuscript (2 ECTS)

Theoretical Ecology: modelling species fitness in competitive environments (2013)

- Aquatic Ecology: how response diversity affects alternative stable states in lakes and ponds (2015)

\section{Deficiency, refresh, brush-up courses (1.5 ECTS)}

- Basic statistics (2012)

\section{Competence strengthening / skills courses (1.8 ECTS)}

- Scientific writing; WGS (2015)

\section{PE\&RC Annual meetings, seminars and the PE\&RC weekend (0.9 ECTS)}

- PE\&RC Introduction weekend (2012)

\section{Discussion groups / local seminars / other scientific meetings (10.2 ECTS)}

- Lake modelling workshop; Leipzig (2012)

- Aquatic ecology literature discussion group (2012-2015)

- Netherlands annual ecology meeting (2012-2015)

- PE\&RC Food web ecology discussion group (2013-2015)

\section{International symposia, workshops and conferences (6.9 ECTS)}

- Symposium for European freshwater sciences; oral presentation; Munster, Germany (2013)

- Shallow lakes conference; oral presentation (1 ${ }^{\text {st }}$ price); Antalya, Turkey (2014)

- Lake modelling workshop; oral presentation; Driebergen (2015)

\section{Lecturing / supervision of practicals / tutorials (7.5 ECTS)}

- Practical aquatic ecology and water quality (2012-2014)

- Field practical hydrology, water quality and meteorology (2013)

- Computer practical water quality; Duflow/SOBEK (2013-2015)

- Masterclass on the use of ecosystem models in water quality management (2015)

\section{Supervision of a MSc student ( 3 ECTS)}

- Determining the critical flow velocity for resuspension in ditches 


\section{Colophon}

The research presented in this thesis was conducted at the Department of Aquatic Ecology of the Netherlands Institute of Ecology (NIOO-KNAW), in collaboration with the Aquatic Ecology and Water Quality Management Group of Wageningen University.

This research is funded by the Netherlands Foundation for Applied Water Research (STOWA) project no. 443237.

This thesis is NIOO Thesis 135 .

Printed by: Digiforce || Proefschriftmaken.nl

Cover design: Michel Govers (michelgovers@yahoo.com) 